University of Louisville

ThinkIR: The University of Louisville's Institutional Repository

Electronic Theses and Dissertations

8-2014

\title{
Fast and robust hybrid framework for infant brain classification from structural MRI : a case study for early diagnosis of autism.
}

Amir Alansary

University of Louisville

Follow this and additional works at: https://ir.library.louisville.edu/etd

Part of the Electrical and Computer Engineering Commons

\section{Recommended Citation}

Alansary, Amir, "Fast and robust hybrid framework for infant brain classification from structural MRI : a case study for early diagnosis of autism." (2014). Electronic Theses and Dissertations. Paper 24.

https://doi.org/10.18297/etd/24

This Master's Thesis is brought to you for free and open access by ThinkIR: The University of Louisville's Institutional Repository. It has been accepted for inclusion in Electronic Theses and Dissertations by an authorized administrator of ThinkIR: The University of Louisville's Institutional Repository. This title appears here courtesy of the author, who has retained all other copyrights. For more information, please contact thinkir@louisville.edu. 


\title{
FAST AND ROBUST HYBRID FRAMEWORK FOR INFANT BRAIN CLASSIFICATION FROM STRUCTRUAL MRI: A CASE STUDY FOR EARLY DIAGNOSIS OF AUTISM
}

\author{
By \\ Amir Alansary \\ B.S., Mansoura University, Egypt, 2009
}

\begin{abstract}
A Thesis submitted to
J. B. Speed School of Engineering, University of Louisville in Partial Fulfillment of the Requirements

for the Degree of

Master of Science

Department of Electrical and Computer Engineering

University of Louisville

Louisville, Kentucky
\end{abstract}

August 2014 



\title{
FAST AND ROBUST HYBRID FRAMEWORK FOR INFANT BRAIN CLASSIFICATION FROM STRUCTRUAL MRI: A CASE STUDY FOR EARLY DIAGNOSIS OF AUTISM
}

\author{
By \\ Amir Alansary \\ B.S., Mansoura University, Egypt, 2009
}

A Thesis Approved On

June 13th, 2014

Date

by the following Thesis Committee:

Ayman El-Baz, Ph.D., Thesis Director

Jacek M. Zurada, Ph.D., Co-advisor

Dr. Hermann Frieboes 


\section{ACKNOWLEDGEMENTS}

In the Name of Allah the All-merciful, the All-compassionate. All deepest thanks are due to Allah Almighty for the uncountable gifts given to me.

I would like to thank all those people that contributed to the completion of this thesis. I should also mention that this thesis would not have been possible without the help, support, guidance and patience of my thesis advisor, Dr. Ayman El-Baz. I also express my deepest gratitude to Dr. Jacek M. Zurada and Dr. Hermann Frieboes for being on my thesis committee with enthusiasm and taking interest in my research in the midst of many other responsibilities and commitments. And, I would like to thank Dr. Georgy Gimel'farb for his useful discussions and valuable comments and feedback. I want to thank all the people who are part of the research group in the BioImaging Lab, Dr. Ahmed Elnakib, Dr. Fahmi Khalifa, Ahmed Firjani, Matthew Nitzken, Ahmed Soliman, Hisham Sliman, and Mahmoud Mostapha, who have become not only colleagues but also good friends.

Finally, but most important of all, I am grateful to my parents, Mohamed Yehia Alansary and Soheer Anwar Elberashi, my sister Aya and my brothers Ahmed and Omay, who have always given me their unconditional support, encouragement and advice, so that I could concentrate on my thesis. 


\begin{abstract}
FAST AND ROBUST HYBRID FRAMEWORK FOR INFANT BRAIN

CLASSIFICATION FROM STRUCTRUAL MRI: A CASE STUDY FOR EARLY

DIAGNOSIS OF AUTISM
\end{abstract}

Amir Alansary

August 12, 2014

The ultimate goal of this work is to develop a computer-aided diagnosis (CAD) system for early autism diagnosis from infant structural magnetic resonance imaging (MRI). The vital step to achieve this goal is to get accurate segmentation of the different brain structures: white matter, gray matter, and cerebrospinal fluid, which will be the main focus of this thesis. The proposed brain classification approach consists of two major steps. First, the brain is extracted based on the integration of a stochastic model that serves to learn the visual appearance of the brain texture, and a geometric model that preserves the brain geometry during the extraction process. Secondly, the brain tissues are segmented based on shape priors, built using a subset of co-aligned training images, that is adapted during the segmentation process using first- and second-order visual appearance features of infant MRIs. The accuracy of the presented segmentation approach has been tested on 300 infant subjects and evaluated blindly on 15 adult subjects. The experimental results have been evaluated by the MICCAI MR Brain Image Segmentation (MRBrainS13) challenge organizers using three metrics: Dice coefficient, 95-percentile Hausdorff distance, and absolute volume difference. The proposed method has been ranked the first in terms of performance and speed. 


\section{TABLE OF CONTENTS}

Page

ACKNOWLEDGEMENTS

ABSTRACT

LIST OF TABLES

LIST OF FIGURES

iii
iv
viii
ix

\section{CHAPTER}

I INTRODUCTION 1

A Magnetic Resonance Imaging (MRI) . . . . . . . . . . . 3

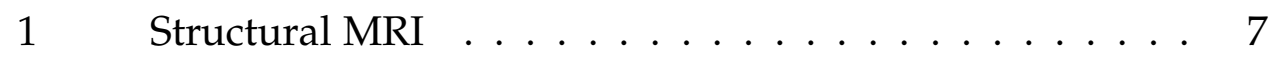

2 Dynamic Contrast-Enhanced MRI (DCE-MRI) . . . . . 9

3 Diffusion MRI (dMRI) . . . . . . . . . . . . . 12

$4 \quad$ Functional Magnetic Resonance Imaging (fMRI) . . . . 15

$5 \quad$ Magnetic Resonance Angiography (MRA) . . . . . . 17

$6 \quad$ Tagged Magnetic Resonance Imaging . . . . . . . . . . 18

7 Magnetic Resonance Spectroscopy (MRS) . . . . . . . . . 19

8 Perfusion-Weighted Imaging (PWI) . . . . . . . . . . . 19

B Computer-aided diagnosis (CAD) System for Autism Diagnosis 21

C Limitations of Existing Work and The Innovation of This Work 23

1 Existing Brain Extraction and Skull Stripping Techniques and Limitations . . . . . . . . . . . . . . . . . 24

2 Existing Brain Tissue Segmentation Techniques and Limitations . . . . . . . . . . . . . . . . . 25

D Thesis Organization $\ldots \ldots \ldots \ldots \ldots \ldots \ldots$ 
II BRAIN EXTRACTION AND SKULL STRIPPING

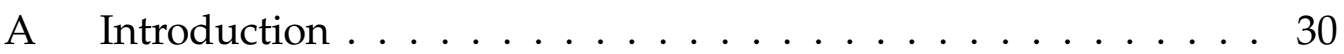

B Methods ..................... 33

1 Bias Correction . . . . . . . . . . . . . . . . . 34

2 Skull Stripping . . . . . . . . . . . . 35

3 Visual Appearance-Guided Iso-Surfaces . . . . . . . . . 36

C Performance Evaluation Metrics . . . . . . . . . . . . . . . 41

1 Dice Similarity Coefficient (D) . . . . . . . . . . . . . 41

2 Modified Hausdorff Distance $\left(\mathrm{H}_{95}\right) \ldots \ldots \ldots$

3 Absolute Volume Difference (AVD) . . . . . . . . . . . 44

D Experimental Results . . . . . . . . . . . . . . . . . 44

E Summary ...................... 48

III BRAIN TISSUE SEGMENTATION 51

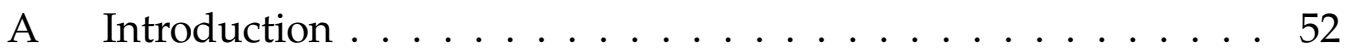

1 Probabilistic segmentation . . . . . . . . . . . 53

2 Atlas-based segmentation . . . . . . . . . . . . 55

3 Deformable models-based segmentation . . . . . . 57

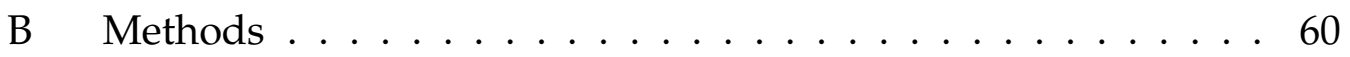

1 First-Order Intensity Model . . . . . . . . . . . . . . 61

2 MGRF Model With Second- and Higher-order Cliques . 61

3 Adaptive Shape Model . . . . . . . . . . . . . . . . . 63

C Experimental Results . . . . . . . . . . . . . . . . 64

D Summary .................... 74

IV CONCLUSION AND FUTURE WORK 77

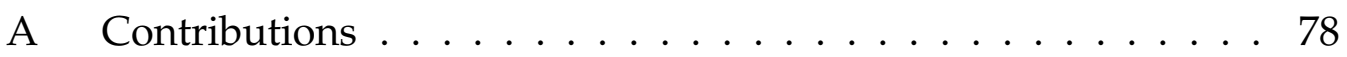

B Future Work . . . . . . . . . . . . . . . . . . . 79 
REFERENCES

A Appendix I - Analytical Estimation of the bi-valued Gibbs Po-

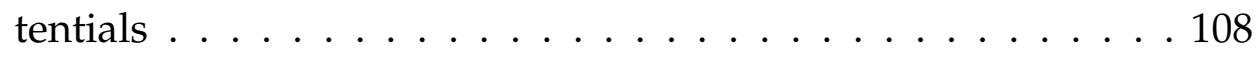

1 Unconditional Region Map Model . . . . . . . . . . 108

2 Identification of the $2^{\text {nd }}$-order MGRF model . . . . . . . 110

B Appendix II - Analytical Estimation of Gibbs Potentials for Higher-Order MGRF Model . . . . . . . . . . . . . . 111

CURRICULUM VITAE

115 


\section{LIST OF TABLES}

TABLE

Page

1 Comparative accuracy of brain extraction approaches table . . . . . 48

2 Detailed adult brain classification results table . . . . . . . . . 69

3 MRBrainS13 challenge summary results table . . . . . . . . . . 71

4 The proposed segmentation approach evaluation results for infant brain table . . . . . . . . . . . . . . . . . . 75

5 Second-, third-, and fourth-order cliques table . . . . . . . . 114 


\section{LIST OF FIGURES}

FIGURE $\quad$ Page

1 Different types of medical images figure . . . . . . . . . . . 2

2 Medical image modalities classification 1 figure . . . . . . . . . 2

3 Medical image modalities classification 2 figure . . . . . . . . 3

4 Different specialized MRI acquisition techniques figure . . . . . . . 5

$5 \quad 2 \mathrm{D}$ MR image of the knee figure . . . . . . . . . . 5

6 Typical 3D MRI of the brain figure . . . . . . . . . . . . 6

7 Typical 4D cardiac MRI data figure . . . . . . . . . . . 6

8 Examples of MRI brain scans figure f . . . . . . . . . . 8

9 MRI scans of the brain using different pulse sequences and scanning parameters figure ...................... 9

10 Dynamic MRI images taken at different time points figure . . . . . . 10

11 Different types of contrast agents figure . . . . . . . . . . 11

12 Typical diffusion MR images for the prostate figure . . . . . . . . 13

13 Colored streamlines represent likely paths of nerve fiber bundles

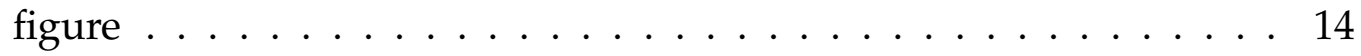

14 Functional MRI image of a normal person reading figure . . . . . . 16

15 Typical TOF-MRA figure . . . . . . . . . . . . . . . . . . 17

16 A typical tagged MRI time-series for the heart figure . . . . . . . . 18

17 A typical MRS image of the brain figure . . . . . . . . . . . . . 20

18 CAD system framework for autism diagnosis figure . . . . . . . . 22

19 Adult and infant MR brain images figure . . . . . . . . . . 26

20 T1-weighted MRI scans for adult and infant brains figure . . . . . . 33

21 Brain extraction framework figure ............... 34 
22 Brain extraction steps figure $\ldots \ldots \ldots \ldots \ldots \ldots$

23 LCDG steps figure . . . . . . . . . . . . . . . . . . . . . . 39

24 Second-order MGRF cliques figure . . . . . . . . . . . . 40

25 Dice similarity (D) calculations figure . . . . . . . . . . . . 43

26 The Haussdorf distance (HD) calculation figure . . . . . . . . . . 44

27 The absolute volume difference (AVD) estimation figure $\ldots \ldots$. . . 45

28 More brain extraction results figure . . . . . . . . . . . 46

29 Comparative brain extraction results figure . . . . . . . . . . . 47

303 D visualization of brain extraction results figure . . . . . . . . . . 49

31 T1-weighted MRI for adult and infant brains figure . . . . . . . . . 52

32 Brain segmentation framework figure . . . . . . . . . . 60

33 Cliques Figure . . . . . . . . . . . . . . . . . . . . 62

34 T1-weighted MR brain images and its normalized empirical density

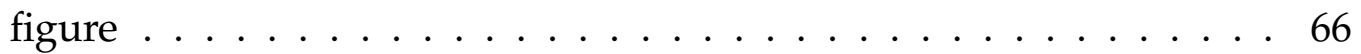

35 Step by step marginal density estimation for each brain tissue class using the LCDG model graphs . . . . . . . . . . . . . . 67

36 2D segmentation results at different cross sections figure $\ldots \ldots 68$

$373 \mathrm{D}$ visualization for the adult brain segmentation results $\ldots \ldots 8$

38 Gray matter segmentation results box-plots . . . . . . . . . . . . 70

39 White matter segmentation results box-plots . . . . . . . . . . 72

40 Cerebrospinal fluid segmentation results box-plots . . . . . . . . . 72

41 Brain segmentation results box-plots . . . . . . . . . . . . 72

42 Intracranial segmentation results box-plots . . . . . . . . . 73

43 3D extracted infant brain figure . . . . . . . . . . . 73

44 Normalized empirical density for infant brain figure . . . . . . . . . 74

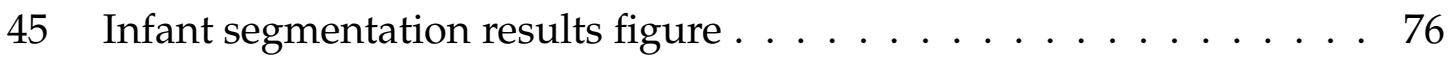




\section{CHAPTER I}

\section{INTRODUCTION}

Medical imaging is the science dealing with techniques and processes for creating visual images of the different body organs for diagnostic and treatment purposes. Medical imaging plays an important role in the improvement of public health due to its ability to provide both anatomical and functional information about the different body organs. Therefore, they can assist radiologists and physicians in disease diagnosis, therapy planning and treatment decisions. There are different imaging modalities and processes to image the body such as structural magnetic resonance imaging (MRI), computed tomography (CT), and ultrasound (US). These modalities enable the acquisition of images for almost all types and sizes of different structures with acceptable degrees of contrast and resolution. Each of these modalities (see e.g., figure 1, figure 2, and figure 3) has its own techniques to find relevant physiological information of the organ being imaged, in addition to its own advantages and drawbacks. Medical images can be classified based on their modalities (figure 2) or based on the type of information that they provide (i.e., the structure or the function of the organ being imaged, see figure 3).

Advances in medical imaging and hardware techniques provide radiologists and physicians with high dimensional (i.e., 3D and 4D) data. Therefore, yielding a great deal of information to be analyzed and evaluated for diseases diagnosis. However, the accurate analysis of this huge data by radiologists is challenging. Therefore, there is an urgent need to develop smart softwares, called computeraided diagnosis (CAD) systems in the medical field, to help the radiologists and 


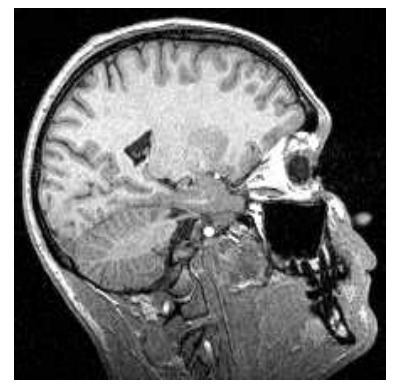

(a)

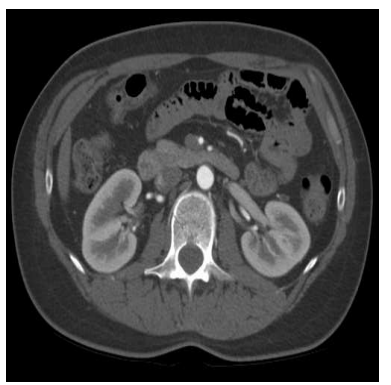

(b)

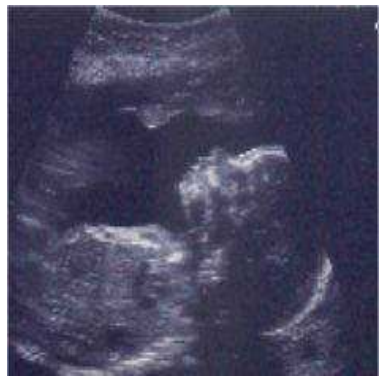

(c)

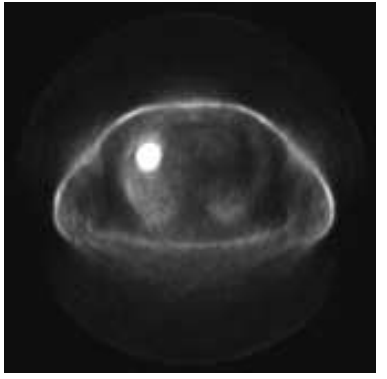

(d)

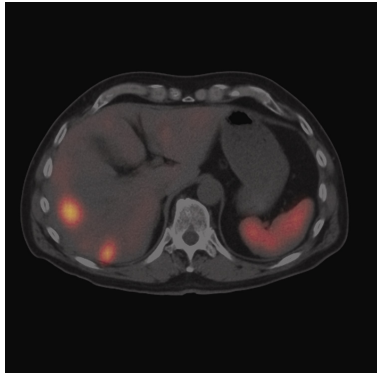

(e)

Figure 1. Different types of medical images: (a) magnetic resonance imaging (MRI) of the brain, (b) computed tomography (CT) image of the kidney, (c) ultrasound (US) image of the fetus, (d) positron emission tomography (PET) image of the lung, and (e) single photon emission computed tomography (SPECT) image of the liver.

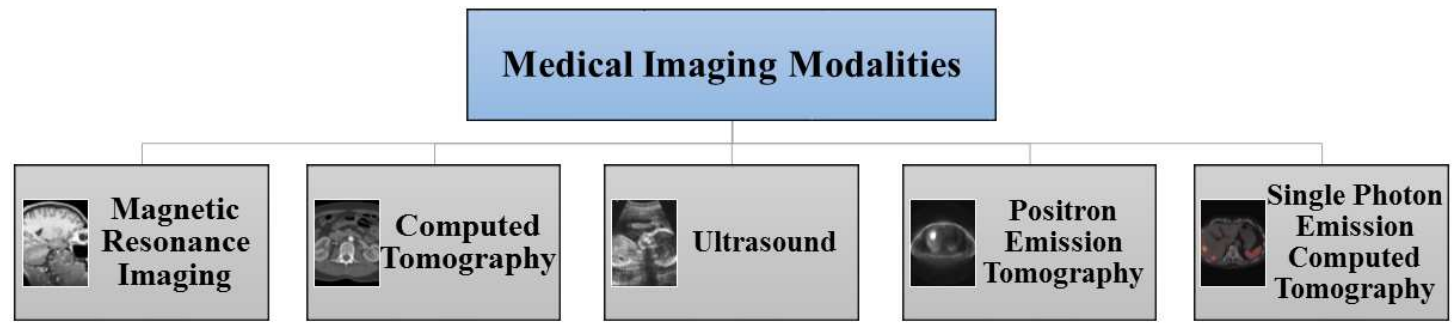

Figure 2. Classes of medical image modalities.

the physicians for accurate and fast diagnosis of diseases. Since, the main focus of the work presented in this thesis is the accurate extraction of brain structures, the best medical imaging modality to describe the brain is structural magnetic resonance imaging (MRI), including (T1-weighted, T2-Weighted, and proton densityweighted MRI, which will be described in more detail in the following section. 
Medical Imaging Modalities

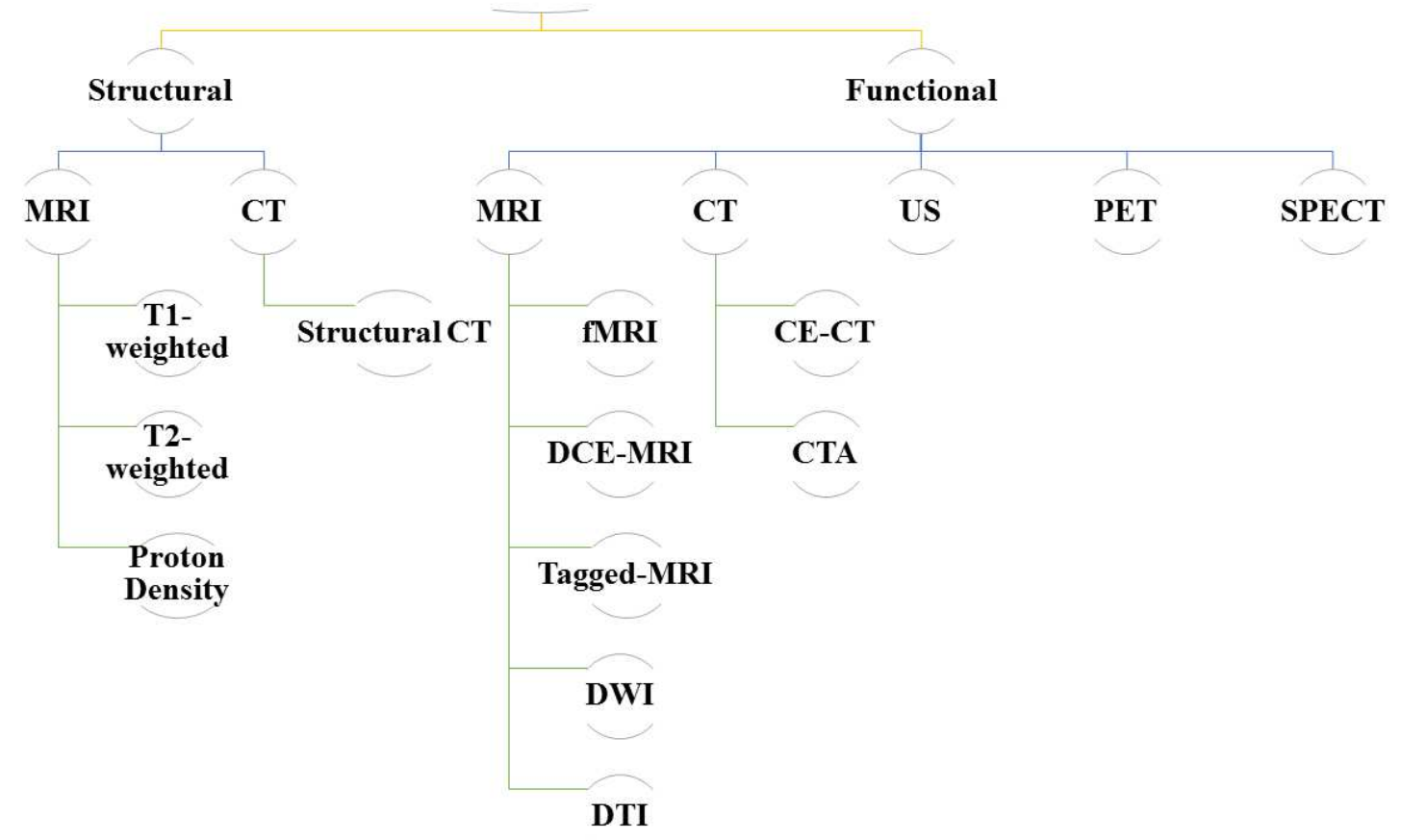

Figure 3. Categories of medical image modalities based on the type of information that they provide about the organ being imaged i.e., structural or functional imaging.

\section{A Magnetic Resonance Imaging (MRI)}

Magnetic resonance imaging (MRI) is an imaging modality used primarily in medical settings to produce high quality images of the inside of the human body, which is based on the principles of nuclear magnetic resonance (NMR) [1]. NMR is a spectroscopic technique used to obtain microscopic physical and chemical information about molecules. The technique was called MRI rather than NMRI because of the negative meaning associated with the word nuclear in the late 1970s. MRI has become the most powerful non-invasive tool for clinical diagnosis of a disease [2]. Fat and water are the primary components in the human body. They contain many hydrogen atoms which make the human body approximately $63 \%$ hydrogen atoms. The main principle of MRI is based on the use of a strong static 
magnetic field in which the hydrogen nuclei in human tissues are aligned parallel to that field. Each nucleus inside the hydrogen atom is comprised of a single proton. The proton possesses a property called spin which: (i) can be thought of as a small magnetic field, and (ii) will cause the nucleus to produce an NMR signal. After using the strong magnetic field, an external radio frequency (RF) pulse is applied to the unpaired magnetic spins (protons) aligned in the static magnetic field, force them to spin in different directions [3]. Energy emission and periodic absorption stem from the interaction between the RF and proton spins. Protons release detectable signals (energy) when they relax back to their lower energy (equilibrium) state. These signals are spatially encoded and are used to construct the MR image. Each tissue type (e.g. muscle, fat, cerebral spinal fluid) send back a different type of tissue-specific signals following the application of the same RF pulse. MR image contrast is strongly dependent on the image acquisition technique. Different components of the scanned area can be highlighted using different pulse sequences: a preselected strength, shape, and timing of defined RF and gradient pulses (external fields). The major advantages of MRI scans are: (i) they can be safely used in people who may be vulnerable to the effects of radiation, such as pregnant women and babies, as they do not involve exposure to radiation, (ii) they are particularly useful for showing soft tissue structures, such as ligaments and cartilage, and organs such as the brain, heart and eyes, and (iii) they allow problems with blood circulation, such as blockages, to be identified, as they can provide information about the blood motions through certain organs and blood vessels. Generally, MRI can be used to acquire planner 2D images (figure 5), 3D volumes (figure 6), or sequences of 3D volumes (i.e., 4D images see, figure 7). Most commonly-known specialized MRI techniques are shown in figure 4 . The different MRI types are explained in more detail in the following section. 


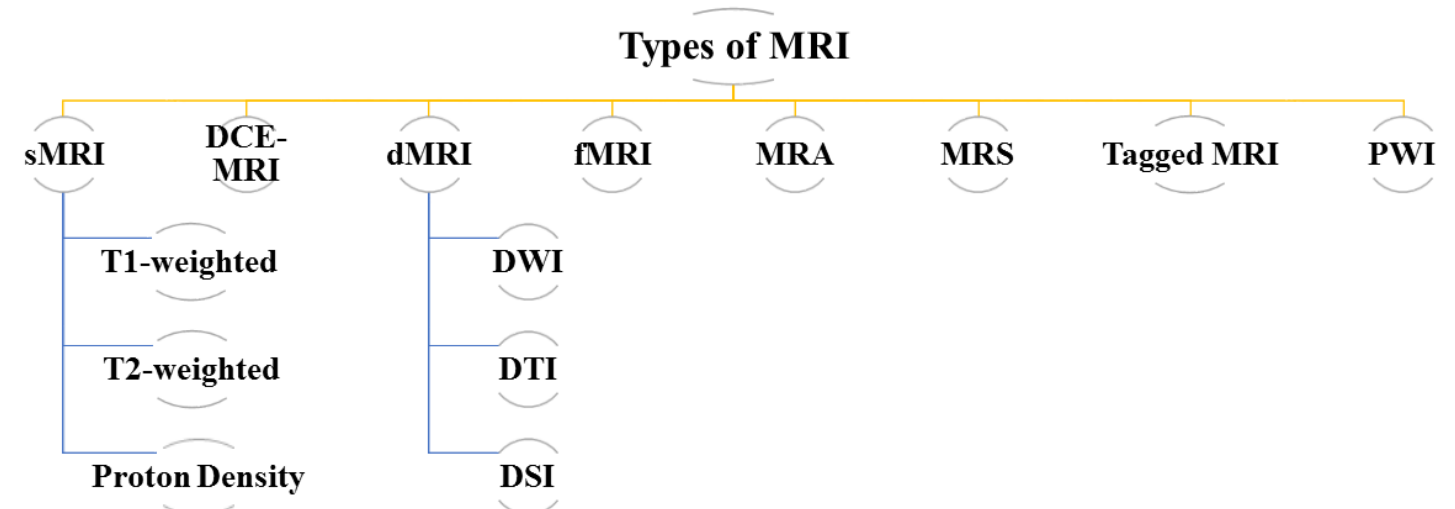

Figure 4. Different specialized MRI acquisition techniques.

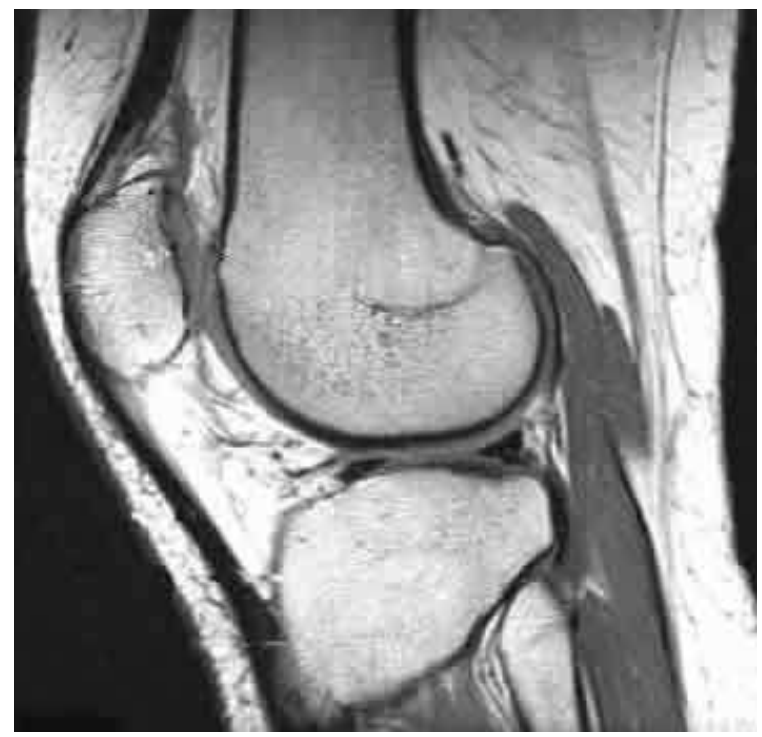

Figure 5. 2D MR image of the knee. Courtesy of [4] 


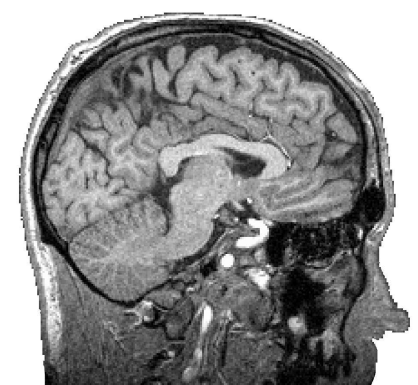

(a)

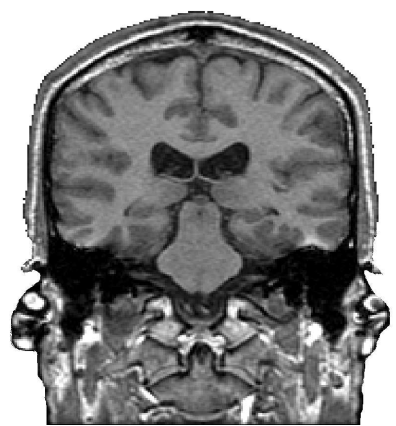

(b)

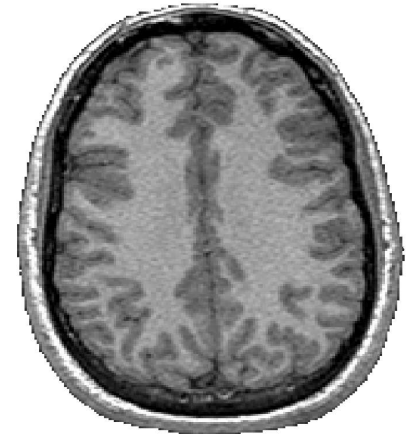

(c)

Figure 6. Typical 3D MRI of the brain, captured in three views: (a) sagittal plane, (b) coronal plane, and (c) axial plane.

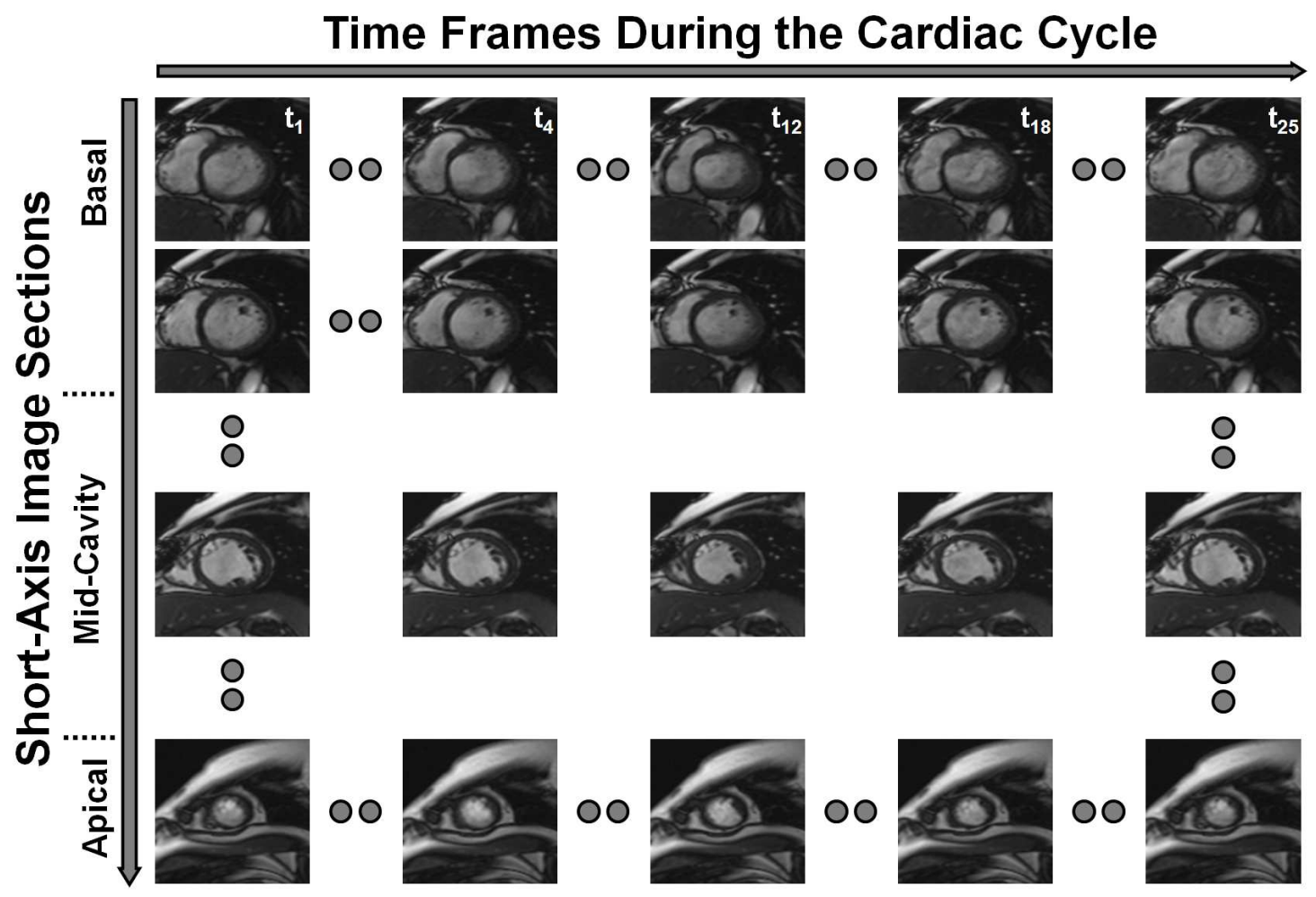

Figure 7. Typical 4D (3D plus time) cardiac MRI data. Images are acquired at different sections covering the heart (from basal to apical) and each section consists of a time series of 25 images over the cardiac cycle. 


\section{Structural MRI}

Structural magnetic resonance imaging (MRI) is a scanning technique for examining the physical structure of the different brain tissues. The amount of energy (or signal strength) on the MRI primarily depends on the magnetic relaxation properties of body atomic nuclei. The time taken by nuclei to return to their baseline states after applying the RF pulse (time of the relaxation process), is known as longitudinal relaxation time (T1) or transverse relaxation time (T2), based on the orientation of the component with respect to the magnetic field. Every human body tissue has its own T1 and T2 values, which depend on proton concentration in this tissue in the form of water and macromolecules.

T1 weighted image (T1-WI) is one of the commonly-run clinical scans based on pulse sequences in MRI, and demonstrates the differences in the T1 relaxation time of the net magnetisation vector (NMV) of tissues, i.e., most of the contrast between tissues is due to differences in tissue T1 values. Fat appears bright on a T1 weighted image as it has a large longitudinal and transverse magnetization. Conversely, water has low signal and appears dark as it has less longitudinal magnetization prior to an RF pulse, and therefore has less transverse magnetization after an RF pulse. Thus, T1-WI is the best MRI method for demonstrating anatomical details.

T2 weighted image (T2-WI) is another type of the basic pulse sequences in MRI and demonstrates the differences in the T2 relaxation time of tissues. Usually, it is used to show high contrast between fluid, abnormalities (e.g., tumors, inflammation, trauma), and the surrounding tissues. Therefore, it is the best MRI method for pathological details. The T2-WI relies upon the transverse relaxation of the net magnetisation vector (NMV). In practice, T1- and T2-weighted images provide complementary information, so both are important for characterizing abnormalities.

Finally, the proton density (spin density) weighted (PD-weighted) scans 


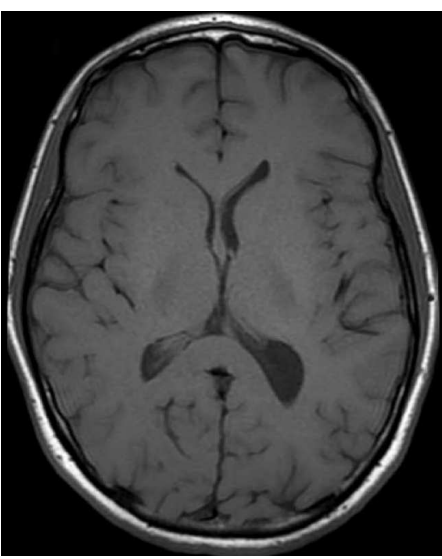

(a)

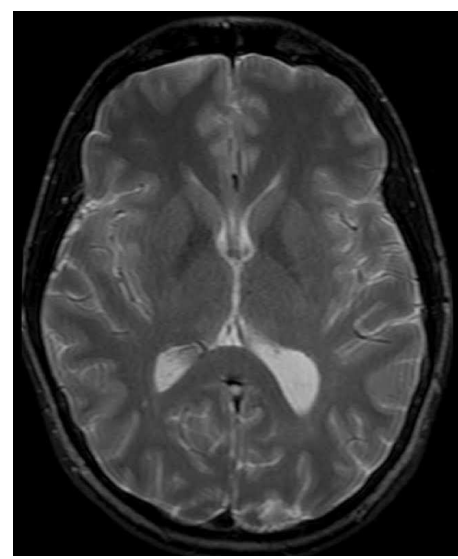

(b)

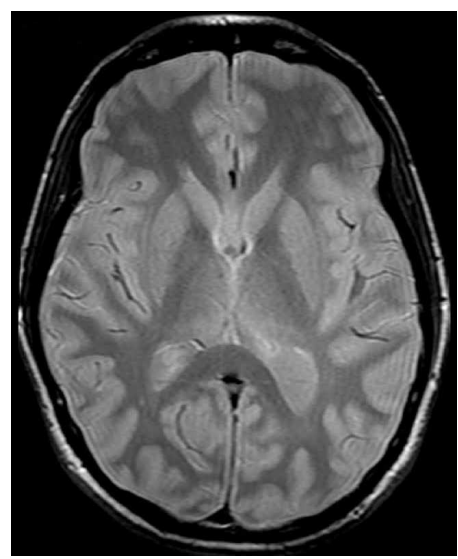

(c)

Figure 8. Examples of MRI brain scans: (a) T1-weighted, (c) T2-weighted images, and (b) proton density. The images have very different image contrasts that reveal specific information about various structures in the brain.

have no contrast from either T1 or T2. The only signal change is due to differences in the amount of available spins (hydrogen nuclei in water). The main advantage of the PD-weighted images is the increase in contrast between fluid and non-fluid tissues. However, PD-weighted images usually show less contrast resolution than T1- and T2-weighted images. This is due to the fact that the difference in hydrogen concentration (proton density) of soft tissues is relatively small. The main strength of structural MRI is that it offers the best soft tissue contrast among all image modalities. Moreover, it is a dynamic technology that can be optimized to tailor the imaging study to the anatomical part of interest and to the disease process being studied. In this regard, structural MRI offers different degrees of dynamic optimization. For instance, the imaging plane can be optimized to the anatomical area being studied (axial, coronal, sagittal, see figure 6), and multiple oblique planes can be captured with equal ease. In addition, as described above, the signal intensities of the imaged tissues can be controlled by selecting the type of the scan: either proton density, T1-weighted, or T2-weighted [2,5,6] (see, figure 8). Moreover, a pulse sequence is designed and imaging parameters are optimized for a given type of scan in order to produce the desired image contrast (see figure 9). 


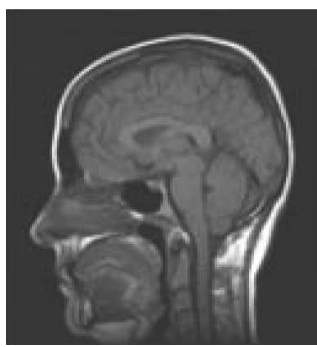

(a)

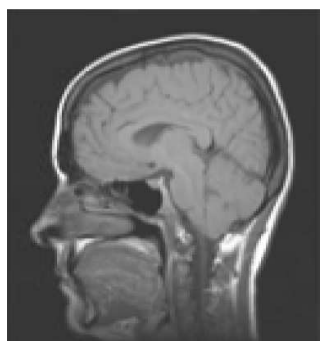

(b)

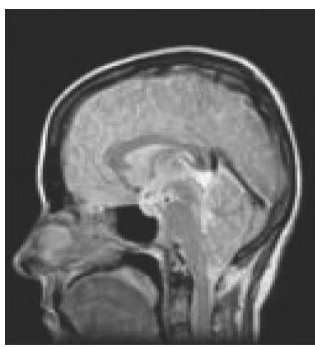

(c)

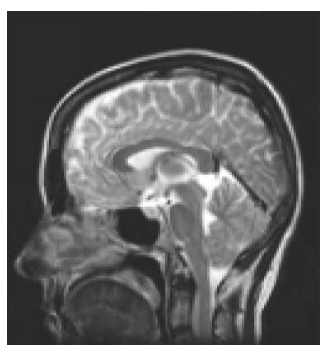

(d)

Figure 9. MRI scans of the brain using different pulse sequences and scanning parameters: (a), (b) two T1-weighted images captured using different scanning parameters and (c), (d) two T2-weighted images captured using different scanning parameters. Courtesy of [7].

\section{Dynamic Contrast-Enhanced MRI (DCE-MRI)}

Although structural MRI gives an excellent soft tissue contrast, it lacks functional information. Dynamic contrast-enhanced MRI (DCE-MRI) is a special MR technique that has the ability to provide superior information of the anatomy, function, and metabolism of target tissues [8]. The DCE-MRI enables analysis of blood vessels generated by a tumor. Its technique involves the acquisition of serial MR images with high temporal resolution before, during, and at several times after the administration of a contrast agent. This contrast agent is blocked by the regular brain-blood-barrier but is not blocked in the blood vessels generated by the tumor. It enables analysis of blood vessels generated by a tumor. In DCE-MRI, the signal intensity in target tissue changes in proportion to the contrast agent concentration in the volume element of measurement (voxel). Also, DCE-MRI is commonly used to enhance the contrast between different tissues, particularly normal and pathological. Figure 10 shows typical examples of dynamic MRI time series data of the kidney, heart, and prostate.

Dynamic MRI has gained significant attention owing to the lack of ionizing radiation, increased spatial resolution, ability to yield information about the hemodynamic (i.e., perfusion) properties of tissues, micro-vascular permeability, 
(a)
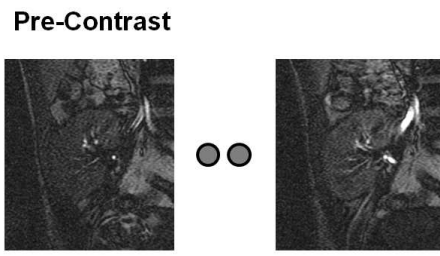

Post-Contrast

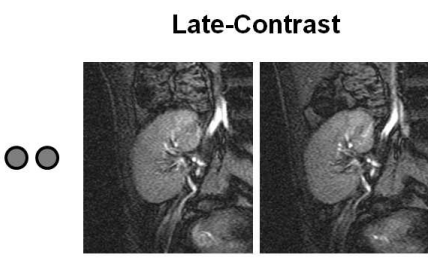

Pre-Contrast
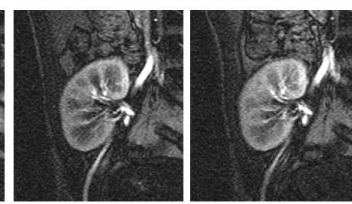

Post-Contrast
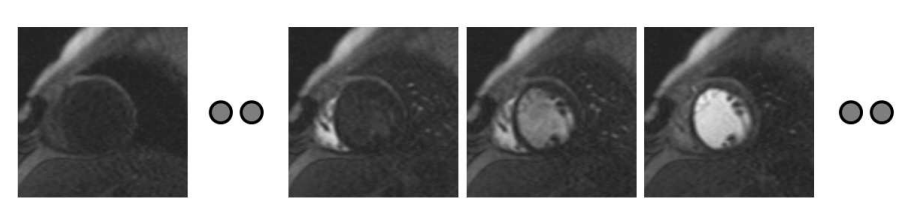

Late-Contrast

Pre-Contrast

Post-Contrast
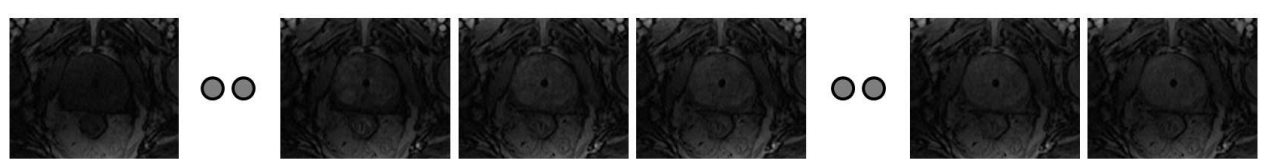

Figure 10. Dynamic MRI images taken at different time points post the adminstration of the contrast agent showing the change of the contrast as the contrast agent perfuse into the tissue beds for (a) kidney; (b) heart, and (c) prostate.

and extracellular leakage space [9]. It has been extensively used in many clinical applications, including detection of pathological tissue, e.g., brain tumors, analysis of myocardial perfusion [10], early detection of acute renal rejection [11-17], and detection of prostate cancer $[18,19]$.

DCE-MRI technique employs the administration (oral, rectal, intravesical, or intravenous) of contrast agents prior to the medical scan, unlike structural MRI where the contrast mainly relies on the intrinsic magnetic relaxation times $\mathrm{T} 1$ and T2. However, T1 and T2 are often too limited to enable sensitive and specific diagnosis due to their intrinsic contrast. In the brain, the widely used clinical agent (e.g., gadolinium) is confined by the blood brain barrier and behaves basically like an intravascular agent. In other tissue beds, such as heart and kidney, gadolinium behaves as a leakage agent and namely distributes in the extra cellular extra vascular space. Parameters can be derived from the reflection of the agent to the tissue bed after short times (up to about two minutes) following the administration of the contrast agent at DCE-MRI. The main role of the contrast agents' 


\section{MRI Contrast Agents}
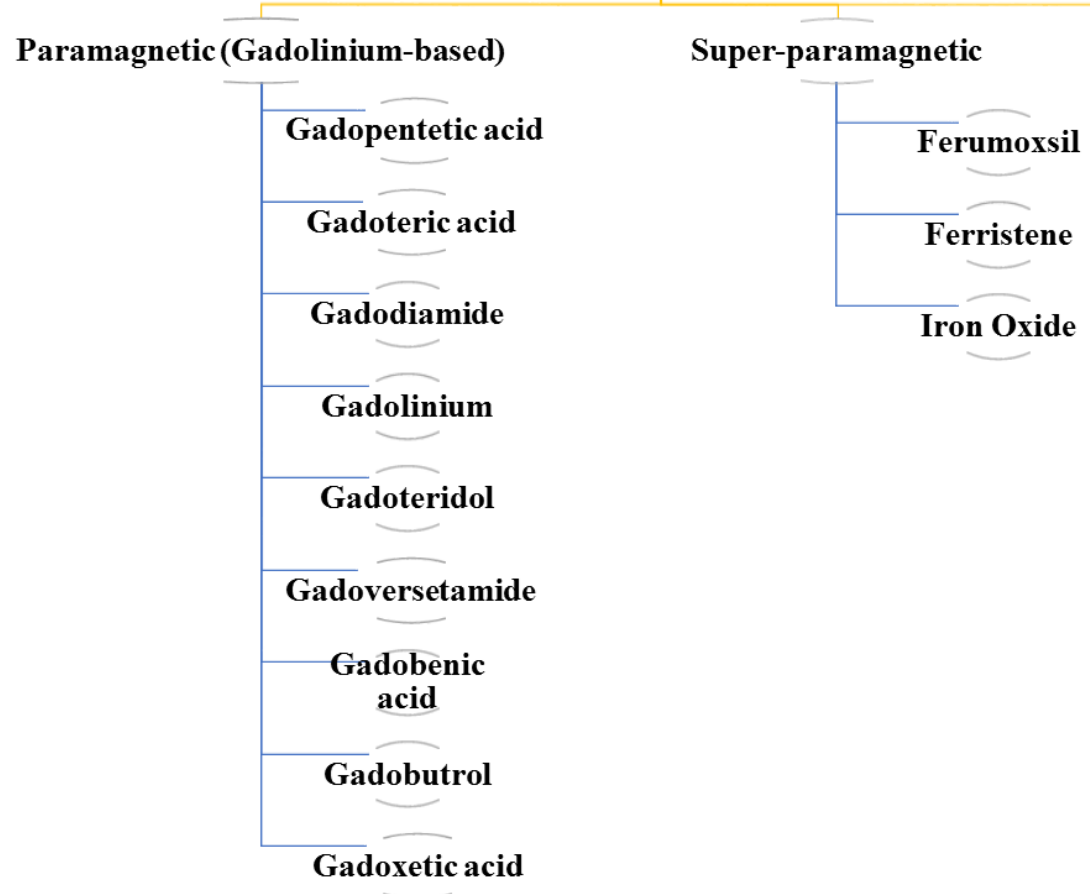

Other Types

Perflubron

Ferristene

Iron Oxide

Figure 11. Different types of contrast agents used in MRI medical scans.

usage is to increase the image contrast of anatomical structures (e.g., blood vessels), which are not easily visualized by the alteration of the magnetic properties of water molecules in their vicinity. This in turn improves the visualization of tissues, organs, and physiological processes. Several types of contrast agents are used in clinical practice and their choice is based on the imaging modality. Specifically, there are several types of contrast agents in MRI such as paramagnetic agents, super-paramagnetic agents, extracellular fluid space (ECF) agents, and tissue (organ)-specific agents as shown in figure 11.

Super-paramagnetic contrast agents are based on water insoluble iron oxide crystals, usually magnetite $(\mathrm{Fe} 3 \mathrm{O} 4)$ or maghemite $(\gamma$-Fe2O3). These contrast agents are suitable for MRI scans of the gastrointestinal tract (GI), such as liver, spleen, esophagus, the stomach, etc. The super-paramagnetic can be classified into super-paramagnetic iron oxide particles (SPIO) and ultra small super- 
paramagnetic iron oxide particles (USPIO) [20]. The gadolinium-based contrast agents are considered as the most successful MRI contrast agents that have been widely investigated. Gadolinium, a rare metal, is a non-toxic paramagnetic contrast agent that enhances the detected MR signal. It produces high contrast images of soft tissues by decreasing T1 relaxation times of water protons in living tissue in the vicinity of the paramagnetic contrast agent. The MRI does not utilize radioactive materials such as high frequency or X-ray in cardiovascular, oncological, and neurological imaging.

\section{Diffusion MRI (dMRI)}

Diffusion MRI (dMRI), a modification of regular MRI techniques, is extensively being used to study the anatomy of the brain and has been an important area of study in the past decade [21]. It offers valuable information about the structure of the human brain that could not be acquired from conventional MRI [22]. dMRI can distinguish water diffusion behaviour in brain tissues, such as anisotropic diffusion in white matter. Tissue segmentation based on dMRI parametric images provides complementary information of tissue contrast to the tissue segmentation based on structural MRI data, which can be employed to define accurate tissue maps when dealing with fused structural and diffusion data [23]. This enables the study of the segmented tissue's diffusivity in neurodegenerative and neurological diseases. More recently, diffusion and functional MRI have emerged in diffusion functional MRI (DfMRI) as it was suggested that could also get images of neuronal activation in the brain from dMRI [24]. Sometimes MRI techniques that depend on contrast agents (e.g., gadolinium-based) are harmful for the body (e.g., patients with kidney problems). Diffusion MRI decrease the severity of using these MRI techniques as it has the advantage of being acquired very rapidly, without the use of any intravenous contrast material or specialized hardware. It is based on the measurement of micromovements (random, Brownian) of extracellu- 
(a)
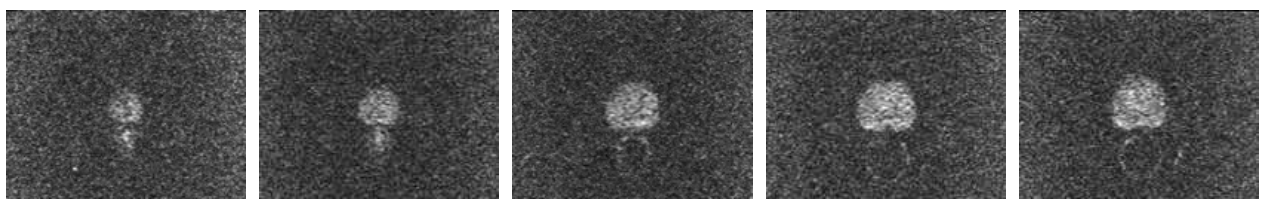

(b)
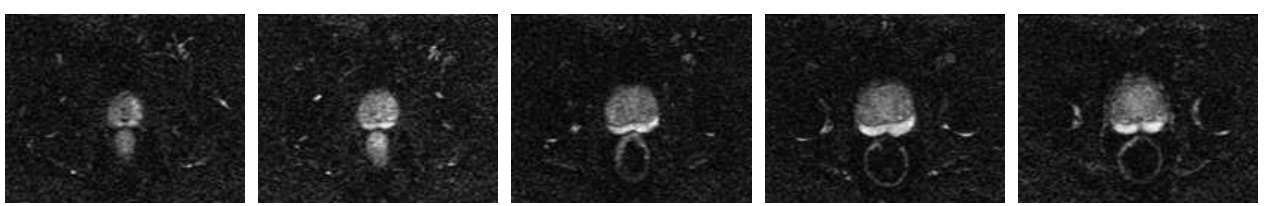

Figure 12. Typical diffusion MR images for the prostate at (a) b-value of $0 \mathrm{~s} / \mathrm{mm}^{2}$ and (b) b-value of $800 \mathrm{~s} / \mathrm{mm}^{2}$.

lar water molecules inside the body. These movements provide indirect information about the structures surrounding these water molecules. Basically, it focuses on the movements of the water molecules inside the body. Diffusion MRI can be classified into three main types, namely, diffusion-weighted imaging (DWI), diffusion tensor imaging (DTI) and diffusion spectrum imaging (DSI).

\section{Diffusion-weighted imaging (DWI)}

Diffusion-weighted imaging (DWI) is used to obtain images whose contrast depends on the differences in water molecule mobility by adding diffusion magnetic field gradients during data acquisition. The b-factor (in $\mathrm{s} / \mathrm{mm} 2$ ) defines the degree of diffusion weighting of the sequence, which depends on the amplitude of the field gradient, time of application, and time interval between the magnetic field gradients. Figure 12 shows a typical DWI-MRI for the prostate. DWI is a well-established MRI method that has been successfully used for tumor localization and diagnosis [25], investigation of brain disorders, such as epilepsy, multiple sclerosis, brain abscesses, brain tumors and hypertensive encephalopathy [26], and in-vivo study of aspects of tissue microstructure [27]. 


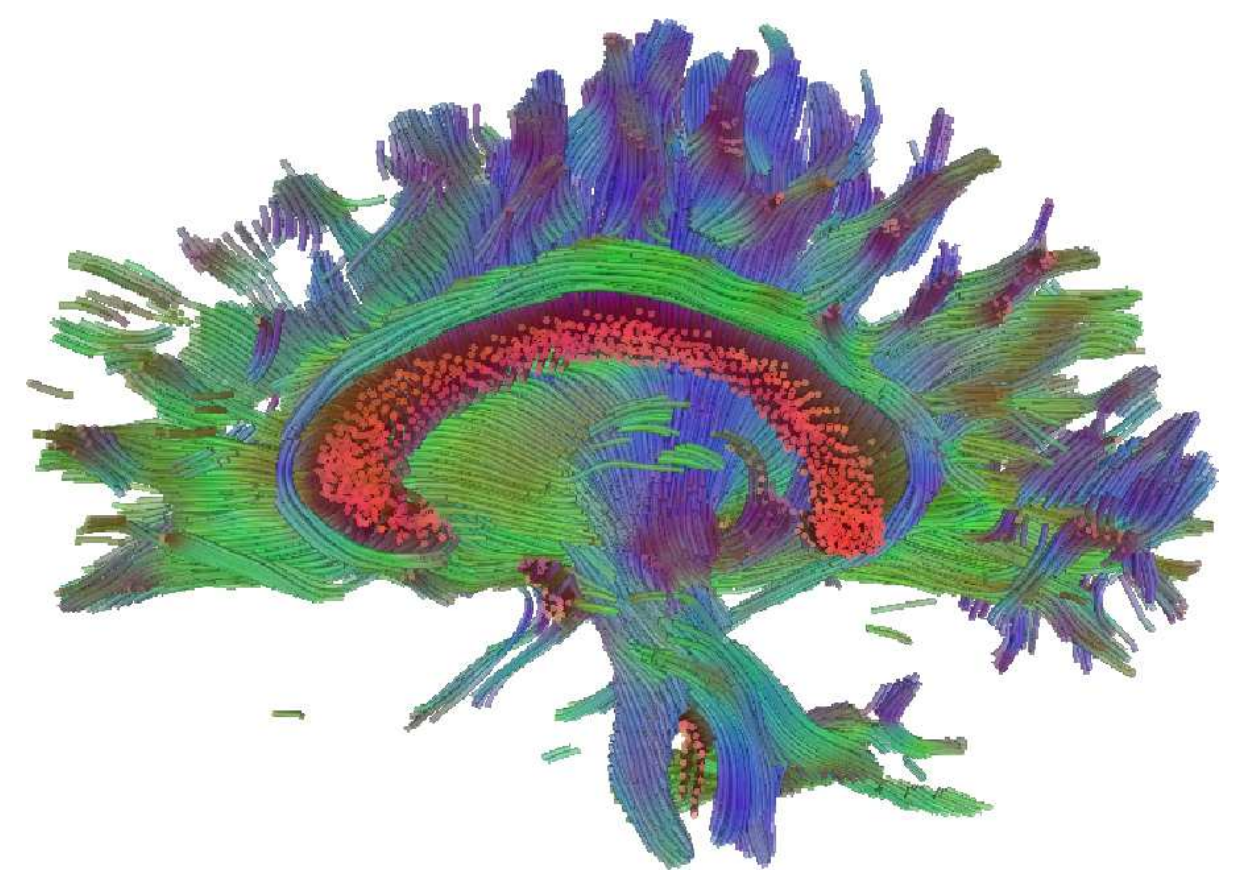

Figure 13. Colored streamlines represent likely paths of nerve fiber bundles. This data was extracted from a diffusion imaging data set. Courtesy of Schultz [29].

\section{Diffusion tensor imaging (DTI)}

Diffusion tensor imaging (DTI) is another diffusion MRI type that is based on the Brownian motion measurements of water molecules in tissue. DTI is a newly-developed MRI technique to study in-vivotissue microstructure (e.g. the connectivity between different brain areas). This MRI modality enables the scientist to look at the network of nerve fibers. Nowadays, DTI has been investigated by neuroscientists to study a number of disorders (e.g., addiction, epilepsy, traumatic brain injury, and various neurodegenerative diseases) and to demonstrate subtle abnormalities in a variety of diseases, (e.g., stroke, multiple sclerosis, dyslexia, and schizophrenia) [26-28]. Figure 13 shows an example of brain nerve's connectivity bundles obtained from a 3D DTI data set. 


\section{Diffusion spectrum imaging (DSI)}

Diffusion spectrum imaging (DSI) is a diffusion MRI technique that is used in deriving the Connectome data sets. Diffusion weighted imaging has been proven as a useful MR technique in studying in-vivo fibrous connectivity. However, it cannot directly image fiber crossings within a single voxel due to its sensitivity to intra-voxel heterogeneities in diffusion directions caused by crossing fiber tracts [30]. To overcome this limitation, Diffusion spectrum imaging (DSI) generalizes the DTI to map complex structures such as crossing fibers. Thus, DSI provides more accurate mapping of axonal trajectories than other diffusion imaging approaches [30]. The disadvantages of DSI are that it requires several hundred images compared with DTI and DWI and requires long acquisition times [31].

\section{Functional Magnetic Resonance Imaging (fMRI)}

Functional MRI (fMRI) extends MRI to detect functional changes in the human organ caused by neuronal activity. Many physicians use fMRI to measure the surgery risk for a patient and to learn how a healthy, diseased or injured organ is functioning [32]. They use fMRI maps to identify areas correlated to critical functions such as speaking, studying, moving, or watching TV. These maps are useful for surgery or radiation therapy planning. Also, many clinicians use fMRI to generate anatomical maps for detecting tumors, stroke, head and injury effects, or diseases such as Alzheimer's [33]. fMRI is used widely in brain to study the activated area of the brain after certain stimuli and to map changes of brain hemodynamics that correspond to mental operations. The technique has the ability to observe brain function as well as which structures participate in specific functions [34]. Functional MRI acquires consequences images, one while the brain is in rest state followed by another one after the brain is stimulated in some way. The areas of brain activation are determined as any regions which are different between the two scans. Functional MRI allows radiologists to better understand brain organi- 


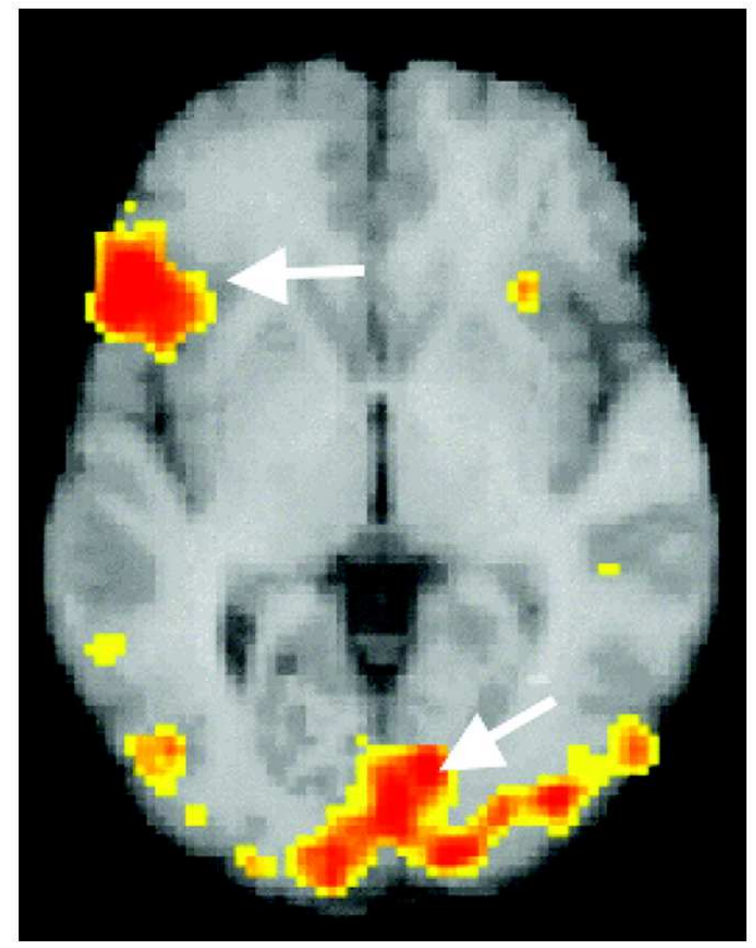

Figure 14. Functional MRI image of a normal person reading. The arrow points to the part of the brain that is activated. Courtesy of Narayana and Xiong [38].

zation and to assess neurological status and neurosurgical risk. Unlike Electroencephalography (EEG) that provides surface information (brain waves) through electrodes mounted on the patients' scalp, fMRI has the advantage of providing in-depth details of what is inside the brain. Clinical applications of fMRI include epilepsy surgery [35], diagnosis of schizophrenia [36], and cerebral injury [37]. A typical fMRI for the brain of a normal person reading is shown in figure 14. The arrows point to parts of the brain that are activated. As demonstrated in the figure, the fMRI can determine the changes in particular regions of the brain in response to a certain stimuli. 


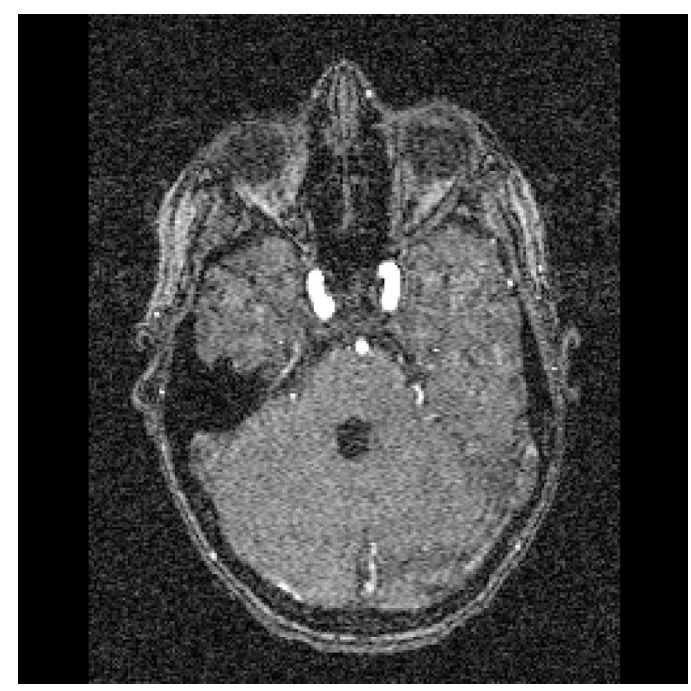

(a)

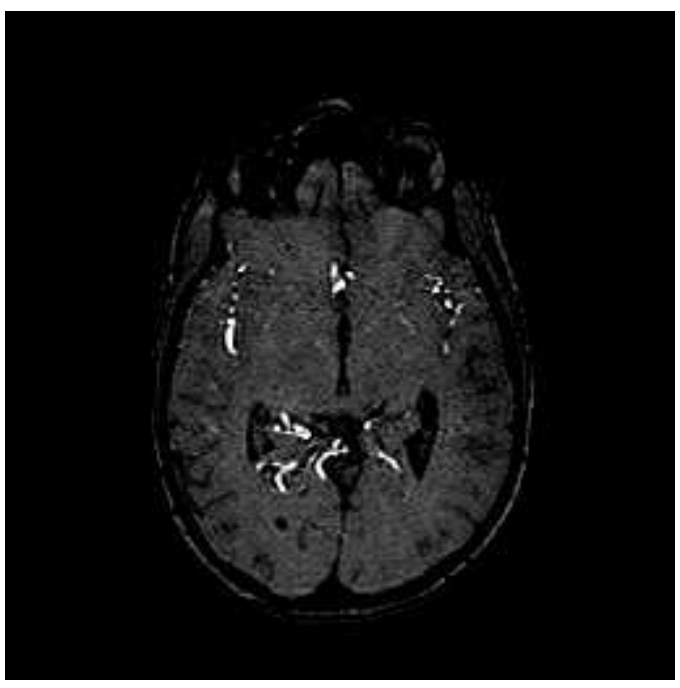

(b)

Figure 15. Typical TOF-MRA (a) and PC-MRA (b) slices.

\section{Magnetic Resonance Angiography (MRA)}

Magnetic resonance angiography (MRA) is an MRI exam for imaging the vascular anatomy using techniques based on magnetic resonance imaging to image blood vessels. MRA is widely used to characterize vascular pathology such as stenosis, dissection, fistula, and aneurysms. Unlike traditional angiography that involves placing a catheter into the body, MRA is considered as a noninvasive scanning technique. Moreover, MRA is a valuable tool in preoperative evaluation of suspected intracranial vascular diseases. MRA can be classified into two major categories: Time-of-flight (ToF) and phase contrast (PC). Both categories are very different technically as they rely on separate physical effects, and result in images with different information about the vasculature [39]. In particular, PC-MRA provides good suppression of background signals and quantifies blood flow velocity vectors for each voxel. On the other hand, TOF-MRA is less quantitative, but it is fast and provides high contrast images. Figure 15 shows an example of 2D TOFand PC-MRA slices of the brain. 


\section{Time Frames During the Cardiac Cycle}

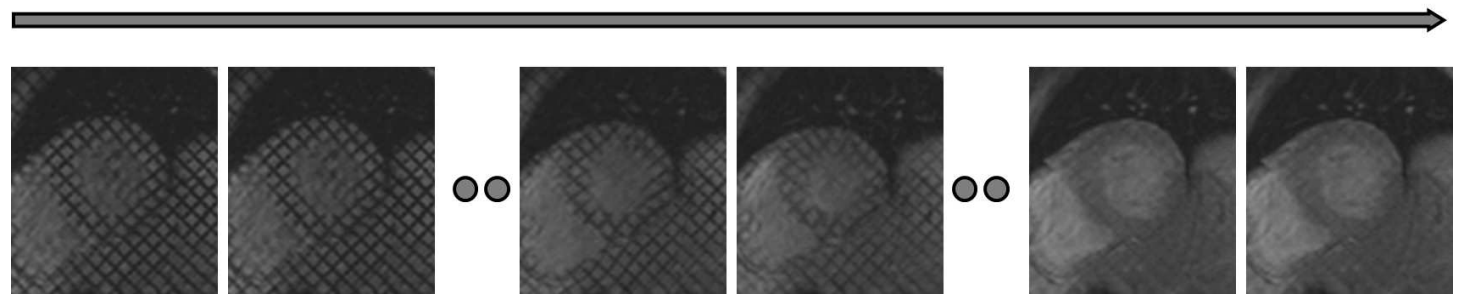

Figure 16. A typical tagged MRI time-series for the heart.

\section{Tagged Magnetic Resonance Imaging}

Conventional magnetic resonance imaging (MRI) has improved the reliability of global cardiac function measurements. However, the lack of reliably identifiable landmarks in the heart wall largely limits tracking the motion of the endocardial or epicardial boundaries. Thus, researchers have developed tagged MRI for detailed and non-invasive visualization of cardiac motions [40]. This imaging modality provides a potentially useful new way to assess the localization of heart diseases (e.g., coronary atherosclerosis) and global conditions (e.g., heart failure and diabetes) that result in heart wall dysfunction. Cardiac MRI tagging places a pre-specified pattern of temporary markers (tags) inside the soft body tissues. These tag lines created by patterns of magnetic spin in the examined tissue so that the motion in the tagged tissue can be measured from the images [41]. This technique extends the traditional anatomical images to capture detailed information about the heart over time. The tag lines allow for computing displacement, velocity, rotation, elongation, strain, and twist of the heart. While traditional MRI techniques carry only information about the motion at the boundaries of an object, the tag lines allow us to examine the strain and displacement of the interior of the tissue in close detail [42]. A typical tagged MRI time-series of the heart is shown in figure 16. 


\section{Magnetic Resonance Spectroscopy (MRS)}

Magnetic resonance spectroscopy (MRS), also known as nuclear magnetic resonance (NMR) spectroscopy, is a non-invasive and ionizing radiation free MRI technique. It has been used to study the chemical activity within cells and to identify the size and stage of a tumor. Unlike conventional MRI that detects the nuclear magnetic resonance spectra of water in tissues, MRS generally detects the resonance spectra of chemical compounds other than water [43]. To allow radiologists to base conclusions on the maximum amount of available information, MRS results are combined with MRI results. MRS has been investigated for diagnosis of patients with brain diseases [44], as it is very useful to study metabolic changes in brain tumors, strokes, seizure disorders, Alzheimer's disease, depression and other diseases affecting the brain. Also, it has been used to study cancerous body organs such as prostate $[45,46]$, breast $[47,48]$, cervix $[49,50]$, pancreas [51], and esophagus [52]. A typical example of an MRS scan for a patient with a brain lesion is shown in figure 17.

\section{Perfusion-Weighted Imaging (PWI)}

Perfusion-weighted magnetic resonance imaging (PWI) is a serial MRI technique designed to image blood flow into brain vasculature. PWI uses a MR contrast (dye) to provide information about the location and extent of cell death within a few hours of a stroke; it can show a decrease in cerebral blood flow. PWI has been shown to be superior to conventional MRI to show blood flow through the blood vessels [54]. Dynamic susceptibility contrast (DSC) is the most common technique used to perfusion-weighted magnetic resonance images. DSC has been thoroughly studied to measure the cerebral blood flow of the brain for patients with vascular stenosis [55], stroke [56], and brain tumors [57]. This MR technique helps the neuroradiologist to more accurately understand brain perfusion by providing other important parameters such as blood volume and perfusion enhancement time. 


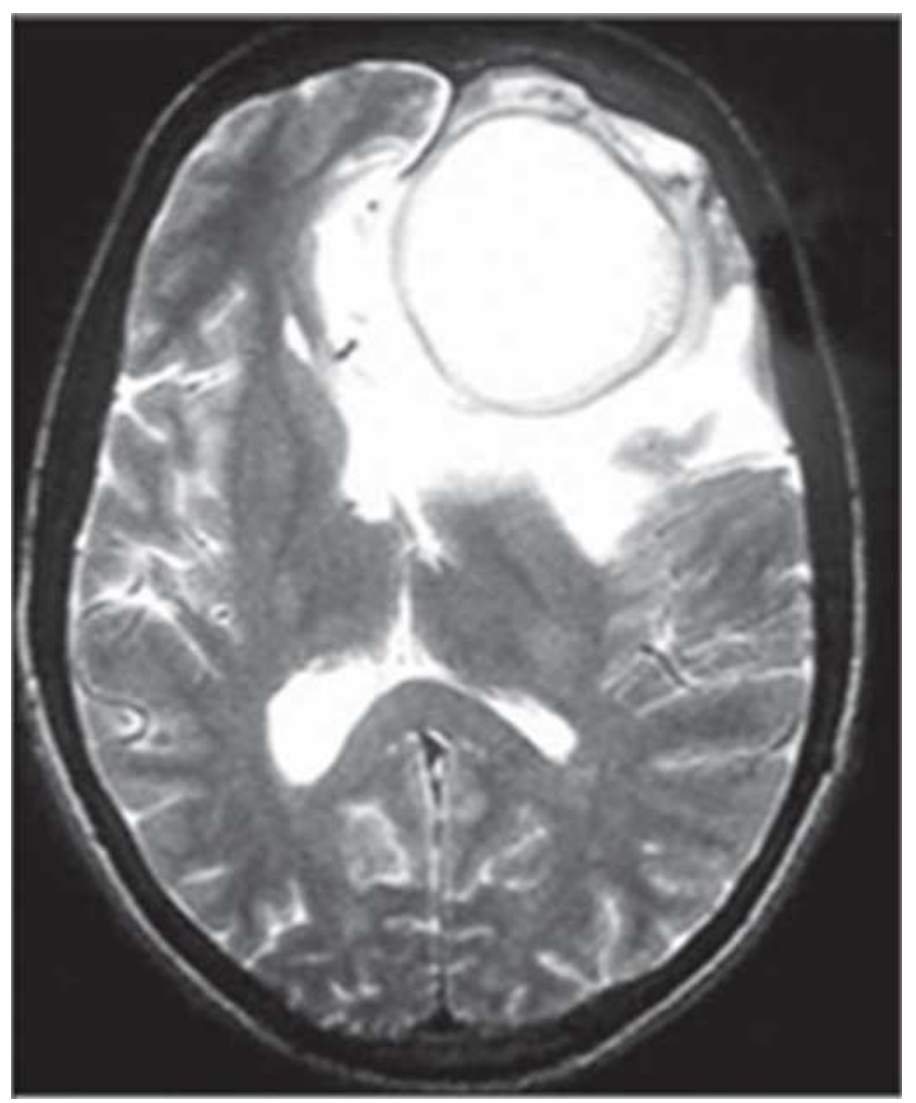

Figure 17. A typical MRS image of the brain. Courtesy of Morais et al. [53]

In total, MRI has a wide range of applications in medical diagnosis. It has many potential advantages: (i) it does not involve exposure to any harmful radiation so they can be safely used in people who may be vulnerable to the effects of radiation, such as pregnant women and babies; (ii) it has the ability to generate cross-sectional images in any plane (including oblique planes); (iii) it can be repeated sequentially over time; (iv) it provides superior resolution with far better contrast (the ability to distinguish the differences between two arbitrarily similar but not identical tissues) compared with other medical image modalities [2]; (v) it is useful for showing soft tissue structures, such as ligaments and cartilage, and organs such as the brain, heart and eyes; (vi) it provides information about the blood motion through certain organs and blood vessels, allowing problems with 
blood circulation, such as blockages, to be identified; and (vii) it plays an important role in assessing tumors' locations and extent, directing biopsies, planning proper therapy, and evaluating therapeutic results [58].

On the other hand, MRI has its own disadvantages: (i) its data acquisition is a relatively long and complex process-it is needed to fix the imaging parameters and the pulse sequence for each scan; (ii) it is not suitable for patients with metal implants due to its magnetic nature; (iii) it suffers from sensitivity to noise and image artifacts; (iv) MRI signals are dependent on the imaging sequence used and can become non-linear beyond certain concentrations leading to errors in extracted physiology, and $(v)$ MRI scanning processes may be uncomfortable for some people because it can produce claustrophobia. Recent improvements in MRI design aim to aid claustrophobic patients by using more open magnet designs and shorter exam times. However, there is often a trade-off between image quality and open design. The next section shows a computer-aided diagnosis system based on structural MRI.

\section{B Computer-aided diagnosis (CAD) System for Autism Diagnosis}

Autism is a developmental disorder characterized by social deficits, impaired communication, and restricted and repetitive patterns of behavior. Currently, there are no medical exams to precisely diagnose autism. Doctors depend on observation, and talking with parents, physicians and therapists about the child in question to make a diagnosis. Thus, developing CAD systems for autism diagnosis is a hot point of research. The ultimate goal of the proposed work in this thesis is to develop a CAD system to classify autistic from normal brains, which is shown in figure 18. This CAD system consists of three main steps: (i) infant brain tissue classification from medical images, (ii) extraction of discriminatory features (e.g., shape features, WM thickness, cortical volume, etc) for the segmented brain tissues, and (iii) classification of autistic from normal infant brains based on ana- 


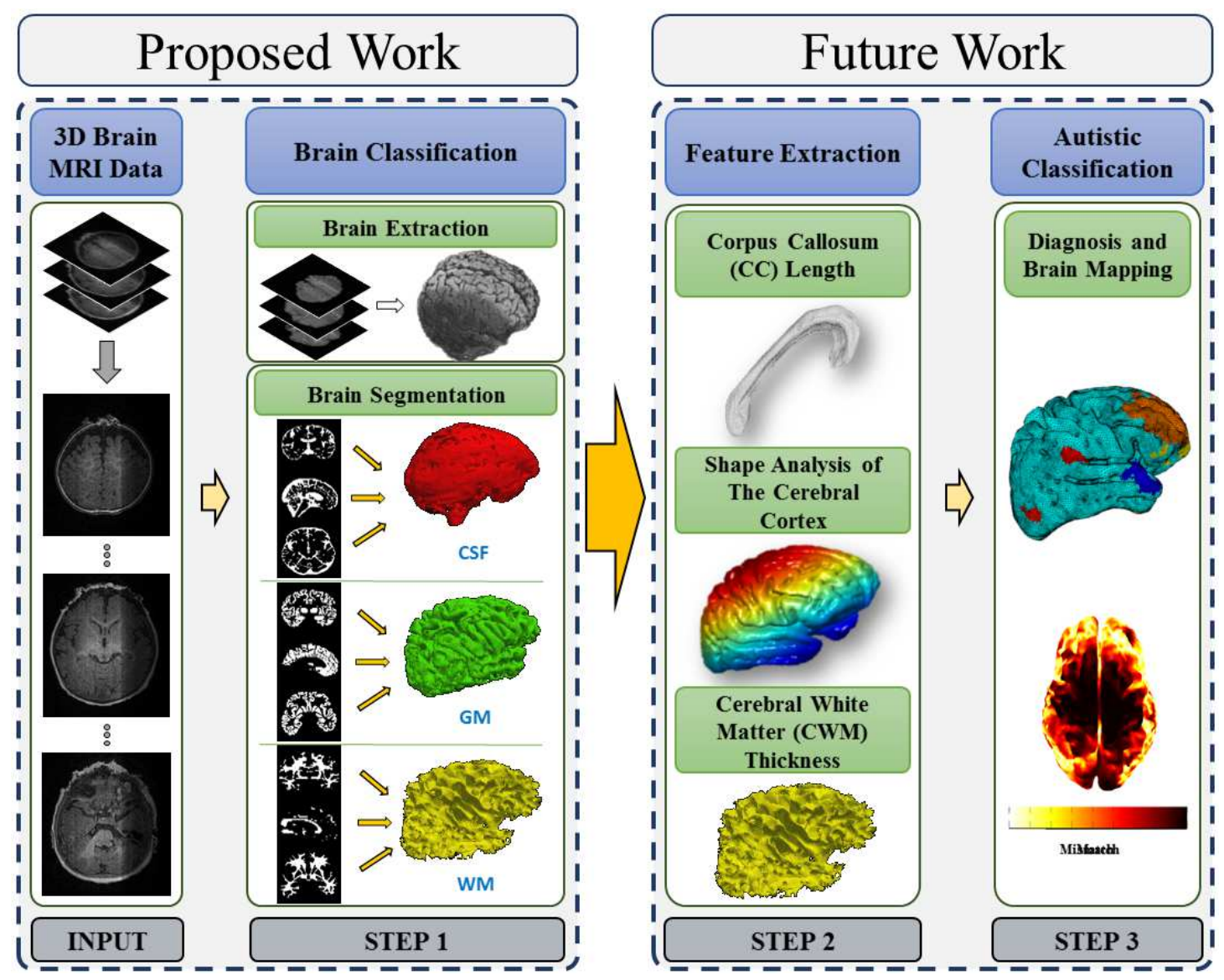

Figure 18. The basic steps of the proposed CAD system framework for autism diagnosis from infant MR brain data.

lyzing the extracted features and shapes of different brain tissues for both normal and autistic brains. This thesis emphasizes the first step in this CAD system, which is developing an accurate and fast infant brain classification framework from structural MRI.

The input to the CAD system is the medical scans of the brain, i.e., structural MR medical images. The first step of a typical CAD system for autism diagnosis is the accurate classification of the infant brain tissue from the input MRI data. This step consists of: (i) brain extraction and skull stripping, i.e., removing outer tissues, e.g. eyes, dura, and skull from the input brain data; and (ii) segmenting the extracted brain into different tissues such as WM, GM, CSF, etc. Fol- 
lowing tissue segmentation, the next step in the autism CAD system is to extract discriminatory features, which are numerical values that correspond to attributes of the segmented region (e.g., WM thickness, shape indexes, corpse callosum (CC) length, volumetric-based metrics). The extraction of appropriate features for brain classification is an essential, yet challenging research area. Recent neuropathological studies show an increasing evidence that children diagnosed with autism spectrum disorder have anatomical differences from controls in cortical volume (CV) [59]. Another study [60] observed differences between the autism and control subjects in total gray matter volumes over time with significantly greater decreases in the autism group compared with control subjects, in addition to the differences in cortical thickness (CT) over time with decreases in the autism group compared with control subjects in several brain regions including the frontal lobe. Other studies [61] used spherical harmonic analysis to describe the shape complexity of the brain and identifies autistic and control brains based on the number of harmonics that can be used to approximate the brain cortex. Finally, the extracted features will be used to distinguish between autistic and normally developed brains based on one of the state of the art classifiers. These classifiers can be categorized into two types: machine learning-based classifiers such as deep learning, random forest and decision tree; and statistical-based classifiers such as bayesian, $k$-nearest and neural network. Moreover, this final step may involve advanced stages, e.g. identification of brain regions that have significant differences between autistic and control subjects using constructed brain maps.

\section{Limitations of Existing Work and The Innovation of This Work}

Since this thesis focuses on the accurate segmentation of the brain tissue from structural MRI, the brain tissue segmentation approach is divided into two major steps: (i) brain extraction and skull stripping, and (ii) brain tissue segmentation. In the literature, a tremendous number of brain extraction and tissue seg- 
mentation techniques have been proposed for the segmentation of different brain tissues from structural MRI. Next, an overview of the existing techniques for brain extraction and brain tissue segmentation and their own shortcomings is provided.

\section{Existing Brain Extraction and Skull Stripping Techniques and Limitations}

Brain extraction is the process of removing all the outer tissues (e.g. eyes, dura, scalp, and skull) around the brain, which consists of the gray matter (GM) and white matter (WM), while the inclusion of cerebrospinal fluid (CSF) in the brain depends on the application. Different brain extraction approaches have been developed; however, they have their own drawbacks. Some of them give better results when removing non-brain tissue while losing some brain parts, and others give better results when extracting the whole brain while keeping some non-brain tissue parts $[62,63]$. For instance, in cortical thickness estimation, inaccurate skull stripping (e.g. failing to remove the dura or missing brain parts) can result in an overestimation or underestimation of the cortical thickness [64]. Atlas-based approaches are very time consuming and their performance heavily depends on the registration accuracy between the atlas and the test subject, in addition to the difficulty of constructing an infant brain atlas [65]. Moreover, the majority of the existing techniques are developed to work for adult MR brain images and fail to accurately extract the brain from MR infant images due to the reduced contrast and higher noise [66]. The infant brain MRI extraction meets with challenges stemming from image noise, inhomogeneities, artifacts, and discontinuities of boundaries due to similar visual appearance of adjacent brain structures.

To overcome the aforementioned limitations, this thesis presents a hybrid framework that possesses the ability to accurately extract brain tissue from infant MR brain images. The proposed framework is primarily based on the integration of a stochastic model (a two-level Markov-Gibbs random field (MGRF)) that serves to learn the visual appearance of the brain texture, and a geometric model 
(the brain iso-surfaces) that preserves the brain geometry during the extraction process. This framework integrates both stochastic and geometric approaches and consists of four basic steps: (i) bias correction, (ii) skull stripping, (iii) iso-surfaces generation, and (iv) final brain extraction using the visual appearance features of the MR brain images. Details of the proposed approach are outlined in Chapter II.

\section{Existing Brain Tissue Segmentation Techniques and Limitations}

Brain tissue segmentation is the process of segmenting the extracted brain into different brain tissues, e.g. WM, GM and CSF. Accurate brain tissue segmentation from MRI is an essential step in clinical diagnostics, therapy evaluation, human brain mapping, and neuroscience [67]. In particular, segmenting an infant brain MR image contributes much to the analysis and treatment of brain injury and disorder resulting from the infant brain prematurity [68]. However, the brain MRI segmentation meets with challenges stemming from image noise, inhomogeneities, artifacts, such as partial volume effect, and discontinuities of boundaries due to similar visual appearance of adjacent brain structures. This thesis targets the infant brain MRI segmentation, which is more complicated than the adult brain segmentation, which may be based on only image intensity. The intensity-based segmentation methods rely on the contrast between different types of tissues and high signal-to-noise ratios. Thus it is hindered by reduced contrast, higher noise from infants [66], and inverse contrast between the White Matter (WM) and Gray Matter (GM) in the infant brain MRIs [69] as shown in figure 19. A large variety of segmentation techniques have been developed for the last two decades in order to address the brain MRI segmentation challenges. These techniques can be roughly classified into three main categories: (i) probabilistic, or statistical methods, (ii) atlas-based methods, and (iii) techniques based on deformable models.

Statistical-based techniques are easier to implement compared to other segmentation methods. However, they depend only on predefined probability models 


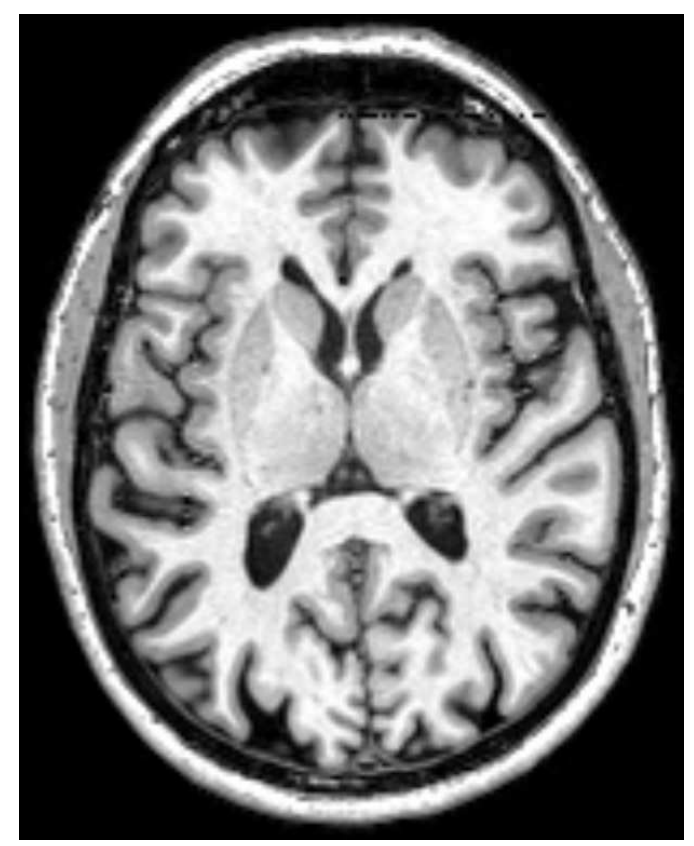

(a)

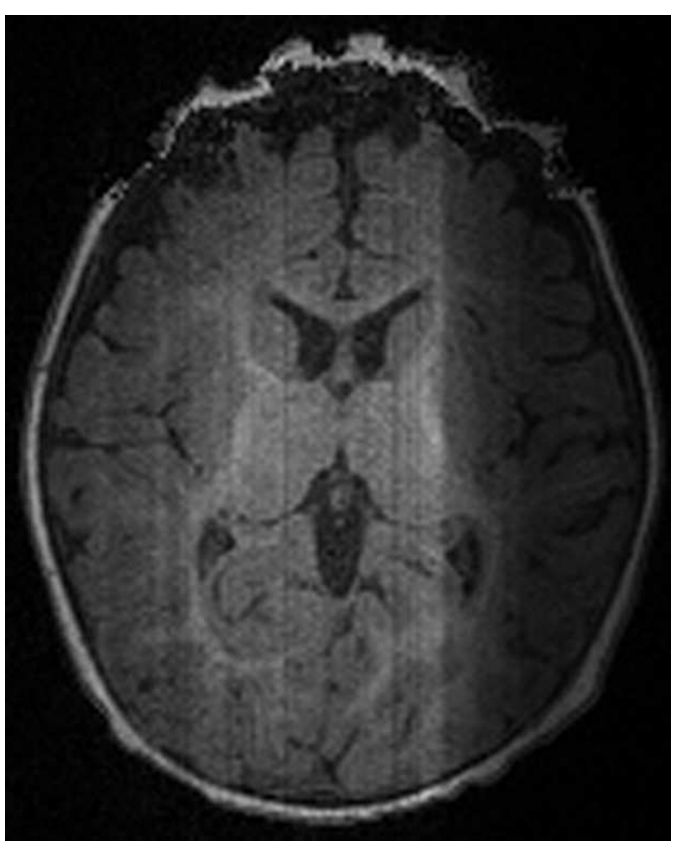

(b)

Figure 19. T1-weighted MRI scans for adult (a) and infant (b) brains.

that cannot fit all of the possible real data distributions. This is due to the fact that actual intensity distributions of brain structures are greatly affected by several factors, such as the unique patient and scanner along with scanning parameters. Also, due to the similar intensities (gray levels) for the different brain tissue structures of the infant MR brain images, segmentation techniques only based on the intensity remain inaccurate.

Atlas-based segmentation techniques show more accuracy with respect to statistical-based techniques. Nevertheless, they are still challenged by atlas selection, combination, and the associated heavy computation time. Another major drawback of atlas-based segmentation algorithms is their dependency on the selected features that will be used to link between the test subject and the prior (training) data used in the construction of the atlas. For example most of the cur- 
rent techniques use signal intensity to find the correspondence between the data to be segmented and the prior atlas. This may lead to inaccurate segmentation results as signal intensities (gray levels) vary due to many factors, such as age, patient and scanner.

Deformable model-based segmentation techniques have the ability to segment connected (non-scattered) objects more accurately than the other segmentation methods. However, the accuracy of this method is based on the accurate design of the guiding forces (statistical, geometric, etc.) in addition to the initialization of the model.

In summary, current segmentation techniques for infant brain MRIs suffer several drawbacks. While statistical-based techniques may be quickly implemented, they depend on predefined probability models that are not capable of fitting all possible real data distributions that arise from uniqueness in patients and variations in scanners and scanning parameters. Additionally, segmentation techniques based on the intensity remain inaccurate due to similar intensities between infant brain structures.

To overcome the aforementioned limitations, the proposed brain tissue segmentation framework is based on prior shapes built using a subset of co-aligned training images that is adapted during the segmentation process based on firstand second-order visual appearance characteristics of infant MRIs. This model is combined with a novel fourth-order MGRF spatial interaction model. These adaptive probabilistic models increase the segmentation accuracy by accounting for large inhomogeneities in infant MRIs and by reducing the effects of noise. Details of the proposed approach are outlined in Chapter III.

\section{Thesis Organization}

This thesis consists of four chapters. The following remarks summarize the scope of each chapter: 
- Chapter I presents some basic concepts about medical imaging and structural MRI, a brief summary of the basic contributions of the proposed research for infant brain extraction and classification from structural MRI, and an overview about the current existing techniques and their limitations.

- Chapter II presents a novel framework for the automated extraction of the infant brain from T1-weighted MR images, which is a crucial step before brain tissue classification that is demonstrated in more details in Chapter III. The proposed approach is primarily based on the integration of a stochastic model (a two-level Markov-Gibbs random field (MGRF)) that serves to learn the visual appearance of the brain texture, and a geometric model (the brain iso-surfaces) that preserves the brain geometry during the extraction process.

- Chapter III presents a new framework for the segmentation of different brain structures from 3D infant MR brain images. The proposed segmentation framework is based on a shape prior built using a subset of co-aligned training images that is adapted during the segmentation process based on firstand second-order visual appearance characteristics of infant MRIs. These characteristics are described using voxel-wise image intensities and their spatial interaction features.

- Chapter IV presents a general discussion about the presented research and its results, followed by the main conclusions and the future work. 


\section{CHAPTER II}

\section{BRAIN EXTRACTION AND SKULL STRIPPING}

As demonstrated in Chapter I, the main focus of this thesis is the accurate classification of the infant brain tissue from structural MRI. To achieve this goal, this Chapter proposes a novel framework for the automated extraction of the brain tissues from T1-weighted MR images, which is a crucial step before brain tissue segmentation that will be demonstrated in more detail in Chapter III. The proposed approach is primarily based on the integration of a stochastic model (a two-level Markov-Gibbs random field (MGRF)) that serves to learn the visual appearance of the brain texture, and a geometric model (the brain iso-surfaces) that preserves the brain geometry during the extraction process. The proposed framework consists of four main steps: (i) Following brain intensity normalization, a new 3D MGRF having a 26-pairwise interaction model is applied to enhance the homogeneity of MR images and preserve the 3D edges between different brain tissues; (ii) The non-brain tissue found in the MR images is removed using the brain extraction tool (BET); (iii) The brain is then parceled to nested iso-surfaces using a fast marching level set method; (iv) Finally, a classification step is applied in order to accurately remove the remaining parts of the skull without distorting the brain geometry. The classification of each voxel found on the iso-surfaces is made based on the first- and second-order visual appearance features. The first-order visual appearance is estimated using a linear combination of discrete Gaussians (LCDG) to model the intensity distribution of the brain signals. The second-order visual appearance is constructed by using an MGRF model with analytically estimated 
parameters. The fusion of the LCDG and MGRF, along with their analytical estimation, allows the approach to be fast and accurate for use in clinical applications. The proposed approach was tested on in-vivo data using 300 infant 3D MR brain scans and validated using nine data sets based on three metrics: the Dice coefficient (D), the 95-percentile modified Hausdorff distance $\left(\mathrm{H}_{95}\right)$, and the absolute volume difference(AVD). The experimental results have shown that the proposed approach is capable of outperforming four widely used brain extraction tools: BET, BET2, brain surface extractor (BSE), and infant brain extraction and analysis toolbox (iBEAT).

\section{A Introduction}

Brain extraction is the process of removing all the outer tissues (e.g. eyes, dura, scalp, and skull) around the brain, which consists of the gray matter (GM) and white matter (WM), while the inclusion of cerebrospinal fluid (CSF) in the brain depends on the application. Brain extraction is a primary step in neuroimaging analysis as well as a pre-processing step for many brain analysis algorithms like intensity normalization, registration, classification, and segmentation. Accordingly, accurate brain extraction is crucial for these algorithms to work properly. For instance, in cortical thickness estimation, inaccurate skull stripping (e.g. failing to remove the dura or missing brain parts) can result in an overestimation or underestimation of the cortical thickness [64].

Many brain extraction approaches have been developed to extract the brain from T1-weighted MR brain images. These methods use different techniques, such as, deformable models, atlas-based and label fusion, and hybrid algorithms. Smith [70] developed an automated deformable model-based method, which is widely known as the brain extraction tool (BET). In their approach, the deformable contour is guided by a set of locally adaptive forces, which include morphological and image-based terms in addition to a surface smoothness constraint. Liu et 
al. [71] presented another deformable model-based brain extraction method using a set of Wendland's radial basis functions [72]. Their deformable model is directed by an internal force to consider the smoothness constraint and an external force to impose the intensity contrast across the boundaries. Finally, they integrate the brain contours calculated separately on 2D coronal and sagittal slices to obtain a complete 3D brain volume. Also, Zhuang et al. [73] used a level set-based deformable model that combines two forces: the mean curvature of the curve and the intensity characteristics of the cortex in MR images. Baillard et al. [74] developed a deformable model-based approach to find the brain surface. As an alternative solution for initializing the first contour manually, an atlas-based technique is used to make the brain extraction process entirely automatic. After atlas registration and initial segmentation, the brain is finally segmented based on level sets that use adaptive parameters depending on the input data. Suresh et al. [75] presented a skull stripping approach using graph cuts, which consists of two steps. An initial brain mask is generated using intensity thresholding as a first step. Then, a graph theoretic image segmentation technique is applied to position cuts that isolate and remove narrow connections. Zhaung et al. [76] developed a simple method for brain extraction by estimating image intensity parameters to construct a binary image of the head. Then, an initial contour is estimated. The final brain is extracted using an improved geometric active contour model which extends the solution of the boundary leakage problem to be less sensitive to intensity inhomogeneity. Somasundaram et al. [77] developed a two-stage brain extraction method. First, they locate a region of interest using feature extraction to produce a rough brain segmentation. Then, the final brain segmentation is obtained using morphological operations in three steps: i) erosion, ii) brain area selection, and iii) dilation to generate the final brain mask. Leung et al. [78] presented a brain extraction technique using a template library. Multiple best-matched atlases are selected from comparing the target image to all the atlases in the template library. To segment 
the target image optimally after image registration and label propagation, label fusion techniques are used to combine the labels from different atlases. Iglesias et al. [79] developed a learning-based brain extraction system which combines two models: a discriminative model based on a random forest classifier trained to detect the brain boundary, and a generative model based on finding the contour with highest likelihood according to the discriminative model, which is refined later using graph cuts. Segonne et al. [80] presented a hybrid approach that combines watershed algorithms and deformable surface models. The watershed, with a preflooding height, construct an initial estimate of the brain volume using a single white matter voxel as a global minimum. Then, a surface deformation process is used to correct the initial segmentation inaccuracies. A statistical atlas is used finally to potentially correct the segmentation. Rex et al. [81] developed a metaalgorithm that uses four freely available brain extraction algorithms: brain surface extractor (BSE) [82], brain extraction tool (BET) [70], 3dIntracranial [83], and MRI watershed from FreeSurfer [84]. For extracting the brain, an atlas is used to define which brain extraction algorithm or combination of extractors works best defining the brain in each anatomic region.

In summary, different brain extraction approaches have been developed; however, they have their own drawbacks. Some of them give better results when removing non-brain tissue while losing some brain parts, and others give better results when extracting the whole brain while keeping some non-brain tissue parts $[62,63]$. Atlas-based approaches are very time consuming and their performance heavily depends on the registration accuracy between the atlas and the test subject, in addition to the difficulty of constructing an infant brain atlas [65]. Moreover, the majority of the existing techniques are developed to work for adult MR brain images and fail to accurately extract the brain from MR infant images due to the reduced contrast and higher noise [66]. The infant brain MRI extraction meets with challenges stemming from image noise, inhomogeneities, artifacts, and dis- 
continuities of boundaries due to similar visual appearance of adjacent brain structures, see figure 20. Furthermore, accurate infant brain extraction contributes much to the analysis, treatment, and the early diagnosis of brain injury and disorder resulting from the infant prematurity.

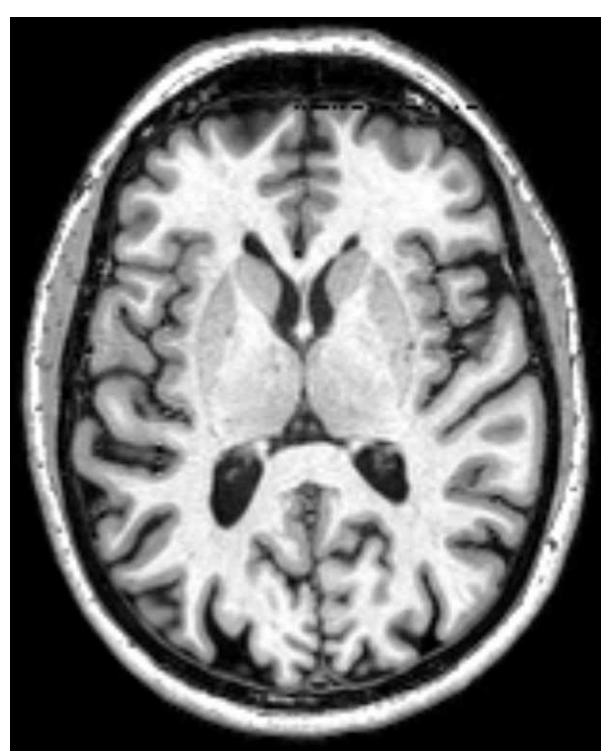

(a)

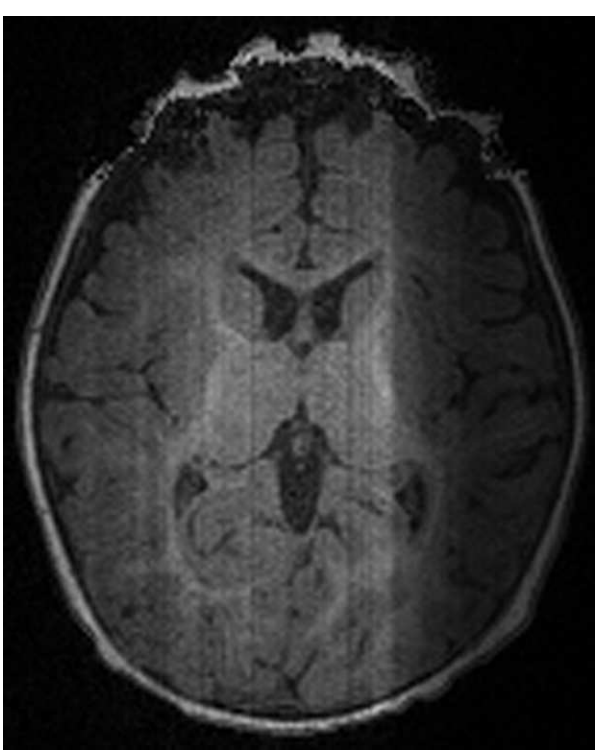

(b)

Figure 20. T1-weighted MRI scans for adult (a) and infant (b) brains.

\section{B Methods}

To overcome the aforementioned limitations, a hybrid framework is presented that is shown in figure 21 , which possesses the ability to accurately extract brain tissue from infant MR brain images. The proposed framework integrates both stochastic and geometric approaches and consists of four basic steps: (i) bias correction, (ii) skull stripping, (iii) iso-surfaces generation, and (iv) final brain extraction using the visual appearance features of the MR brain images. Details of the proposed approach are outlined in the following sections. 

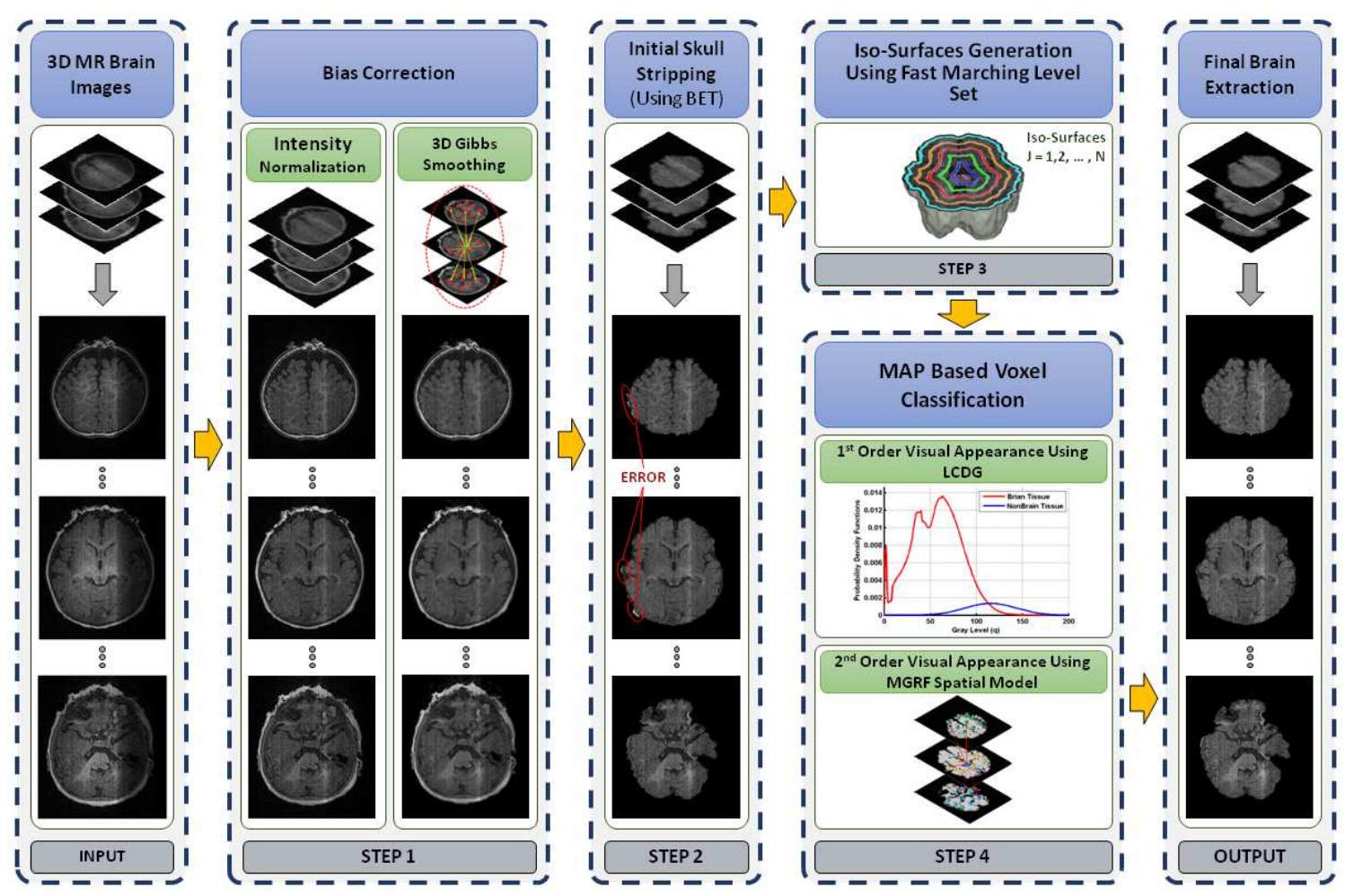

Figure 21. The basic steps of the proposed framework for brain extraction from infant MR brain data.

\section{Bias Correction}

Illumination non-uniformity of infant brain MRIs, which is known as bias field, limits the accuracy of the existing brain extraction approaches. Therefore, to accurately extract the brain it is important to account for the low frequency intensity non-uniformity or inhomogeneity. To achieve this, a 3D generalized GaussMarkov random field (GGMRF) model [85] is applied after brain intensity normalization using the nonparametric approach proposed in [86]. This step reduces noise effects and removes (smooth) inconsistencies of the MRI data by accounting for the 3D spatially homogeneous pair-wise interactions between the gray levels of the MRI data. Namely, the gray level values $q \in \mathbf{Q}=\{0, \ldots, Q-1\}$ are considered as samples from a 3D GGMRF model [85] of measurements with the voxel 26-neighborhood. The continuity of $q$ values of each brain MR scan is amplified by 
using their maximum A posteriori (MAP) estimates [85] and voxel-wise stochastic relaxation (iterative conditional mode (ICM) [87]):

$$
\widehat{q}_{s}=\arg \min _{\widetilde{q}_{s}}\left[\left|q_{s}-\widetilde{q}_{s}\right|^{\alpha}+\rho^{\alpha} \lambda^{\beta} \sum_{r \in \nu_{s}} \eta_{s, r}\left|\widetilde{q}_{s}-q_{r}\right|^{\beta}\right]
$$

where $q_{s}$ and $\widetilde{q}_{s}$ are the original gray level values and their expected estimates, respectively, at the observed 3D location, $s=(x, y, z) ; \nu_{s}$ is the 26-neighborhood system; $\eta_{s, r}$ is the GGMRF potential, and $\rho$ and $\lambda$ are scaling factors. The parameter $\beta \in[1.01,2.0]$ controls the level of smoothing (e.g., $\beta=2$ for smooth vs. $\beta=1.01$ for relatively abrupt edges). The parameter $\alpha \in\{1,2\}$ determines the Gaussian, $\alpha=2$, or Laplace, $\alpha=1$, prior distribution of the estimator. To demonstrate the effect of the first step of the proposed framework, an example of the original, and bias-corrected (intensity normalization and GGMRF edge preservation) brain MR data is shown in figure $22(\mathrm{a})$, and figure $22(\mathrm{~b}, \mathrm{c})$, respectively.

\section{Skull Stripping}

The second step of the proposed framework after the bias correction step is to remove the non-brain tissue from the MR images. To accomplish this step, the conventional BET [70] has been used with a small ' $\mathrm{F}$ ' factor to minimize the loss of brain tissues, which uses a deformable model-based approach to remove the skull from brain MRIs. The initial brain extraction result of figure 22 (c) after the BET is shown in figure $22(\mathrm{~d})$. While the BET extracted the brain without losing any of its parts, it fails to remove all non-brain tissues. For some clinical applications, such as cortical thickness measurement, inaccurate skull stripping results in an over- or under-estimation of the thickness. Therefore, it is important to account for inaccurate skull stripping results after the BET step. 


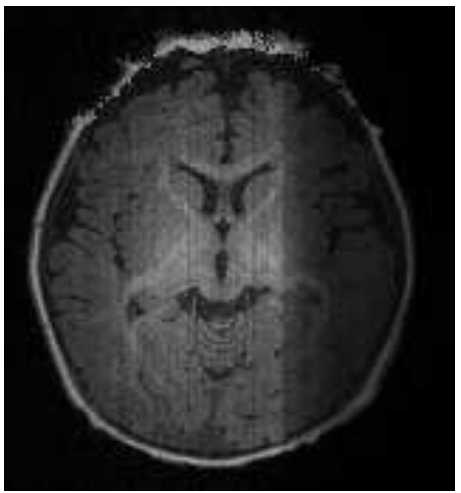

(a)

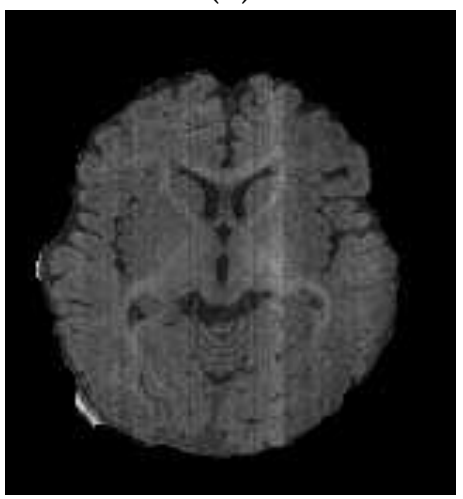

(d)

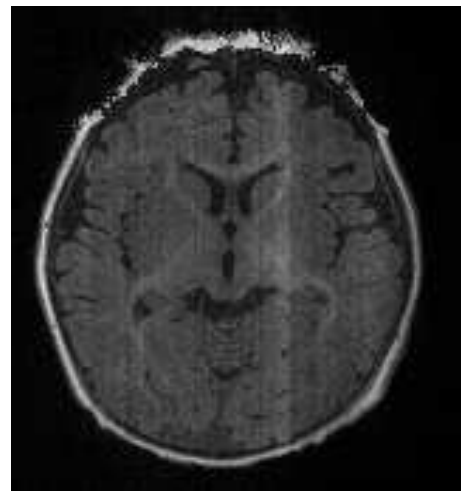

(b)

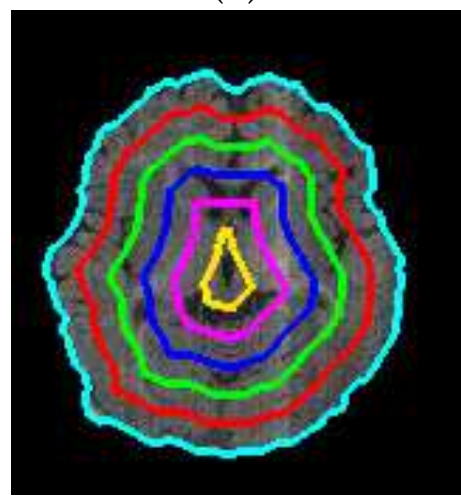

(e)

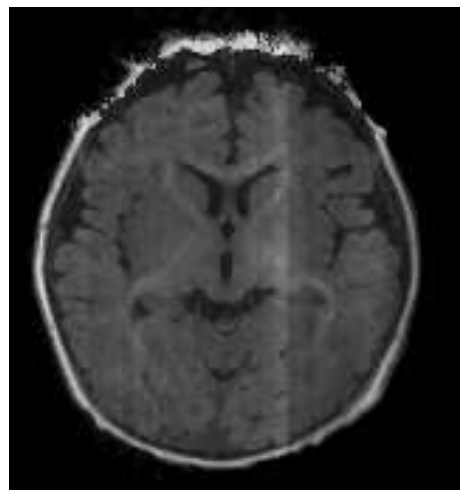

(c)

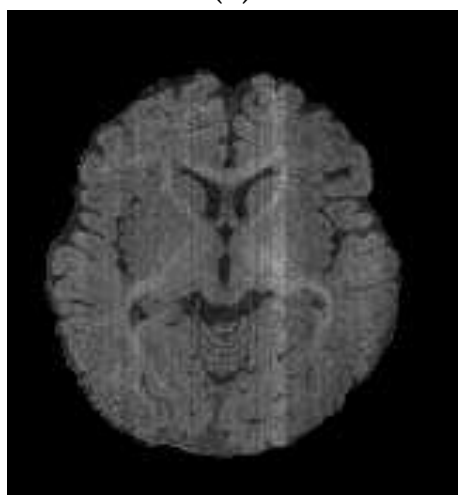

(f)

Figure 22. Step-wise brain extraction using the proposed framework: (a) the original MR image, (b) the bias-corrected image, (c) the GGMRF-edge preserved image obtained with $\rho=1, \lambda=5, \beta=1.01, \alpha=2$, and $\eta_{s, r}=\sqrt{2}$, (d) the extracted brain using BET [70], (e) the iso-surfaces used to remove non-brain tissues, and (f) the final extracted brain.

\section{Visual Appearance-Guided Iso-Surfaces}

In order to obtain more accurate brain extraction results, an additional processing step based on the geometric features of the brain is used to account for BET's skull stripping errors. Since the non-brain tissues are brighter than brain tissue, this step exploits the visual appearance features of the MR brain data. Namely, an evolving iso-surface-based approach is proposed to remove the non-brain tissues, which is guided by the visual appearance features of the MR data (see figure 21, Steps 3 and 4). First, a set of nested, tangent surfaces (i.e., iso-surfaces) are generated by the fast marching level set (FMLS) approach [88], using the extracted 
brain from the BET step. In order to accurately classify MRI voxels as brain or non-brain, a joint Markov-Gibbs random field (MGRF) visual appearance model is used. This MGRF model is described below.

Let $\mathbf{Q}=\{0, \ldots, Q-1\}$ and $\mathbf{L}=\{$ "brain", "non-brain" $\}$ denote sets of gray levels $q$ and region labels $L$, respectively. Let $\mathbf{R}$ denote a 3D arithmetic lattice supporting a given grayscale image $\mathbf{g}: \mathbf{R} \rightarrow \mathbf{Q}$ to be segmented and its goal region map $\mathbf{m}: \mathbf{R} \rightarrow \mathbf{L}$. The 3D T1-weighted MR images, $\mathbf{g}$, and its map, $\mathbf{m}$, are described with the following joint probability model:

$$
P(\mathbf{g}, \mathbf{m})=P(\mathbf{g} \mid \mathbf{m}) P(\mathbf{m})
$$

where $P(\mathbf{m})$ is an unconditional probability distribution of maps, and $P(\mathbf{g} \mid \mathbf{m})$ is a conditional distribution of the images given the map. The ultimate goal is to accurately estimate $P(\mathbf{g} \mid \mathbf{m})$ and $P(\mathbf{m})$, which are described next.

\section{First-order visual appearance $(P(\mathbf{g} \mid \mathbf{m}))$}

To accurately approximate the marginal probability distributions of the brain and non-brain tissue, the empirical gray level distribution of a given brain data is precisely approximated with a linear combination of discrete Gaussians (LCDG) with positive and negative components [89]. The LCDG restores brain and non-brain transitions more accurately than a conventional mixture of only positive Gaussians, thus yielding a better initial map $\mathrm{m}$ formed by voxel-wise classification of the image gray values. Next the LCDG is explained in more details.

Let $\Psi_{\theta}=(\psi(q \mid \theta): q \in \mathbf{Q})$ defines a discrete Gaussian (DG) ${ }^{1}$ where $\theta=(\mu, \sigma)$, integrating a continuous 1D Gaussian density with mean $\mu$ and variance $\sigma^{2}$ over successive gray level intervals [89]. The LCDG with two dominant positive DGs

\footnotetext{
${ }^{1}$ A Discrete Gaussian (DG) $\Psi_{\theta}=(\psi(q \mid \theta): q \in \mathbf{Q})$ with $\theta=\left(\mu, \sigma^{2}\right)$ is defined as $\psi(q \mid \theta)=$ $\Phi_{\theta}(q+0.5)-\Phi_{\theta}(q-0.5)$ for $q=1, \ldots, Q-2, \psi(0 \mid \theta)=\Phi_{\theta}(0.5)$, and $\psi(Q-1 \mid \theta)=1-\Phi_{\theta}(Q-1.5)$ where $\Phi_{\theta}(q)$ is the cumulative Gaussian function with the mean $\mu$ and the variance $\sigma^{2}$.
} 
and $M_{\mathrm{p}} \geq 2$ positive and $M_{\mathrm{n}} \geq 0$ negative subordinate DGs is defined as [89]:

$$
P_{\mathrm{w}, \boldsymbol{\Theta}}(q)=\sum_{i=1}^{M_{\mathrm{p}}} w_{\mathrm{p}: i} \psi\left(q \mid \theta_{\mathrm{p}: i}\right)-\sum_{j=1}^{M_{\mathrm{n}}} w_{\mathrm{n}: j} \psi\left(q \mid \theta_{\mathrm{n}: j}\right)
$$

where all the weights $\mathbf{w}=\left[w_{\mathrm{p}: i}, w_{\mathrm{n}: j}\right]$ are non-negative and meet an obvious con-

straint $\sum_{i=1}^{M_{\mathrm{p}}} w_{\mathrm{p}: i}-\sum_{j=1}^{M_{\mathrm{n}}} w_{\mathrm{n}: j}=1$. All the LCDG parameters, including the numbers of DGs, are estimated from the mixed empirical distribution to be modeled using the modified expectation-maximization (EM)-based algorithm introduced in [90].

The marginal intensity distributions of the MR infant images have two dominant modes: one mode for brain tissues and a second mode for non-brain tissues. Figure 23 illustrates the basic steps to build the LCDG models of both modes. First, the marginal empirical probability distribution of the input grey level images (figure 23(a)) are collected (figure 23(b)). Then, the obtained empirical distribution is approximated with a mixture of two positive DGs relating each to a dominant mode (figure 23(c)). Second, the deviations between the empirical and the estimated distribution (figure 23(d)) are approximated with the alternating "subordinate" components of the LCDG described in [24]. Finally, the obtained positive and negative subordinate mixtures (figure 23(f)) are added to the dominant mixture yielding the final mixed LCDG model (figure 23(g)), which is partitioned into two LCDG-submodels (one per class, figure 23(h)) by associating the subordinate DGs with the dominant terms so that the misclassification rate is minimal [89].

\section{Second-order visual appearance $(P(\mathbf{m}))$}

In order to overcome noise effect and to ensure segmentation homogeneity, the spatial interactions between the region labels of a brain map $\mathbf{m}$ are also taken into account using the popular Potts MGRF model. This model is identified using the nearest voxels' 26-neighbors (as shown in figure (24(a))and analytical bi-valued Gibbs potentials (as shown in figure (24(b)) because only the coincidence of the labels is taken into account. The MGRF model is defined by [91] as: 


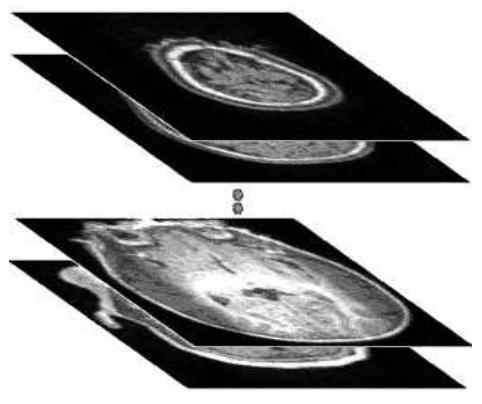

(a)

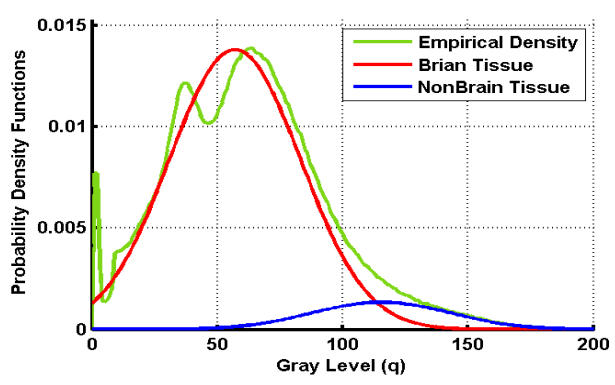

(c)

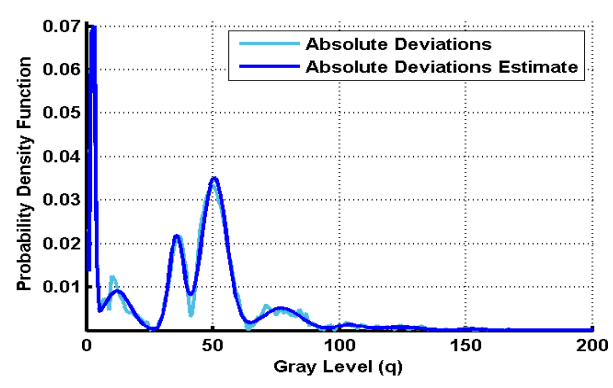

(e)

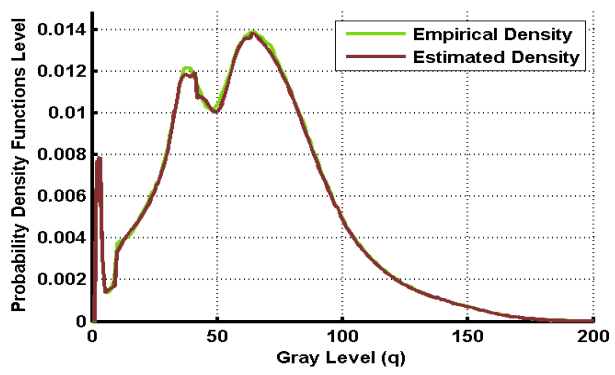

(g)

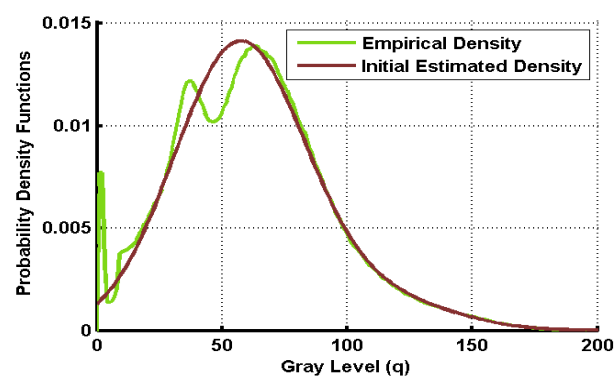

(b)

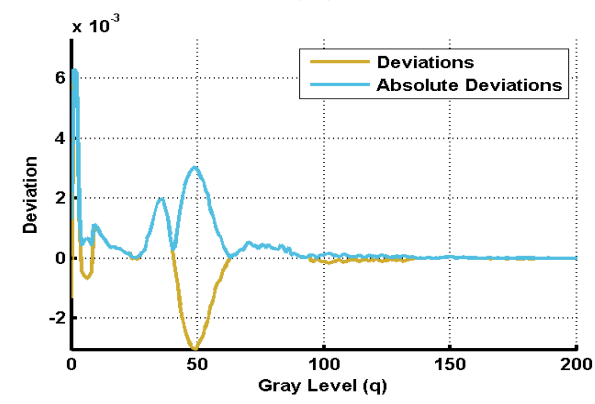

(d)

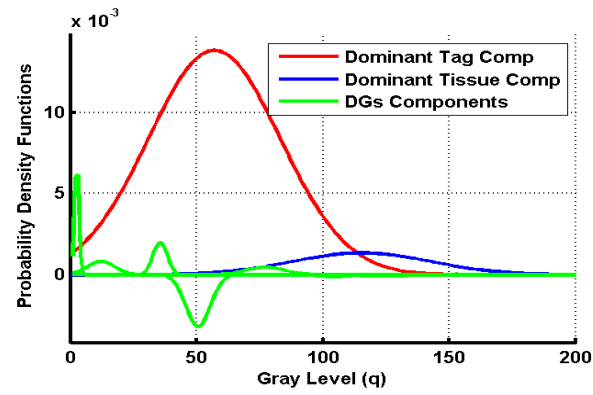

(f)

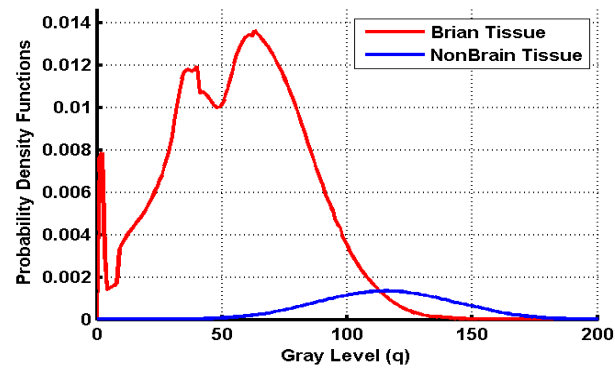

(h)

Figure 23. Typical MR infant brain images (a); and estimated density (b) using only two dominant Gaussian components (c), deviation between empirical and estimated densities (d), estimated density of absolute deviation (e), LCDG components (f), final estimated density (g), and the final estimated marginal density for each class (h). 


$$
P(\mathbf{m}) \propto \exp \sum_{(x, y, z) \in \mathbf{R}} \sum_{(\xi, \zeta, \kappa) \in \nu_{s}} \mathbf{V}\left(m_{x, y, z}, m_{x+\xi, y+\zeta, z+\kappa}\right)
$$

where $\mathbf{V}$ is the bi-value Gibbs potential, that depends on whether the nearest pair of labels are equal or not:

$$
V=\left\{\begin{array}{lll}
V\left(\lambda, \lambda^{\prime}\right)=V_{\mathrm{eq}} & \text { if } & \lambda=\lambda^{\prime} \\
V\left(\lambda, \lambda^{\prime}\right)=V_{\mathrm{ne}} & \text { if } & \lambda \neq \lambda^{\prime}
\end{array}\right\}
$$

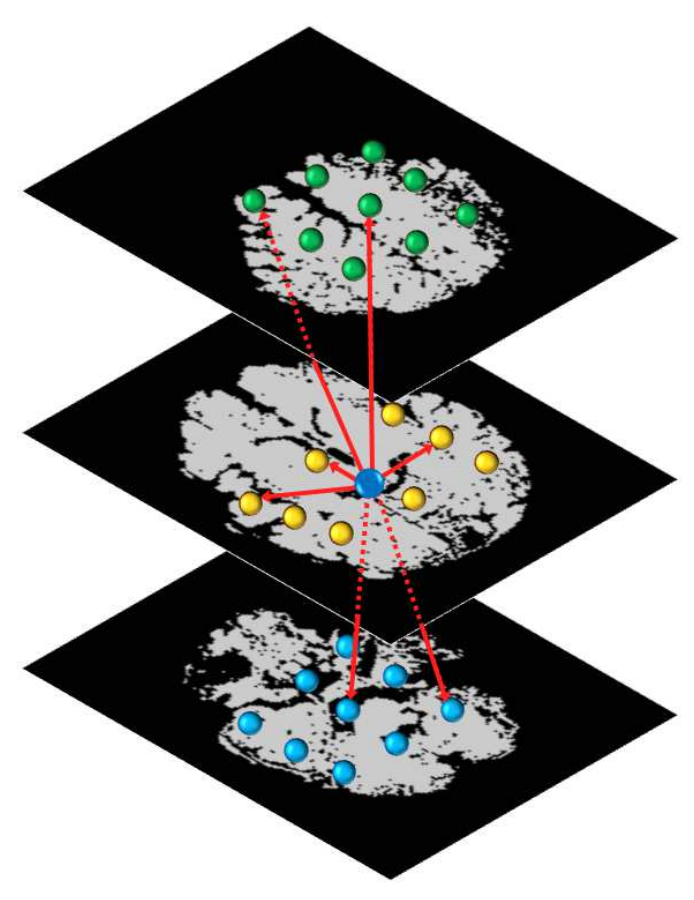

(a)
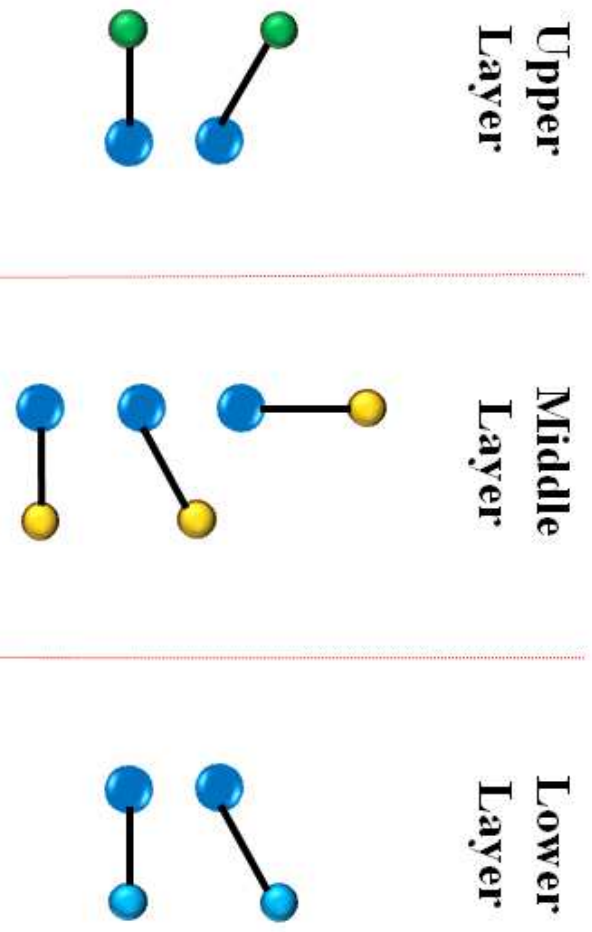

(b)

Figure 24. A graphical illustration for the 3D neighborhood system (a) and a sample of the different pair-wise cliques for the $2^{\text {nd }}$-order MGRF (b).

Let $f_{a \text {,eq }}(\mathbf{m})$ denote the relative frequency of the equal label pairs in the equivalent voxel pairs $\{((x, y, z),(x+\xi, y+\zeta, z+\kappa)):(x, y, z),(x+\xi, y+\zeta, z+\kappa) \in$ $\left.\mathbf{R} ;(\xi, \zeta, \kappa) \in \nu_{s}\right\}$. The initial $\mathbf{m}$ results in approximate analytical maximum likelihood potentials estimates [91]: 


$$
V_{\mathrm{eq}}=-V_{\mathrm{ne}} \approx 2 f_{\mathrm{eq}}(\mathbf{m})-1
$$

that allow for computing the voxel-wise probabilities $p_{x, y, z}\left(m_{x, y, z}=\lambda\right)$ of each label $\lambda \in \mathbf{L}$. In total, Algorithm 1 summarizes the basic steps of the proposed brain extraction framework. For completeness, the analytical estimation of the bi-valued Gibbs potentials is driven in the Appendix.

\section{Performance Evaluation Metrics}

The performance of the proposed framework was evaluated using three performance metrics: (i) the Dice similarity coefficient (D) [92], (ii) the 95-percentile modified Hausdorff distance $\left(\mathrm{H}_{95}\right)$ [93], and (iii) the absolute volume difference (AVD). The following subsections explain the three metrics in more detail.

\section{Dice Similarity Coefficient (D)}

The Dice similarity coefficient (D) characterizes the agreement between the segmented and ground truth objects (as seen in figure 25). The D measure is given from [92] as:

$$
D=\frac{2 T P}{2 T P+F P+F N}
$$

where $\mathrm{TP}, \mathrm{FP}$, and FN denote the true positive, false positive, and false negative respectively. Higher D values indicate better segmentation, which means that the results match the ground truth better than results with lower $D$ values. A D value of 0 indicates no overlap and a $\mathrm{D}$ value of 1 indicates ideal segmentation (or agreement). 
Algorithm 1 Proposed Brain Extraction Approach

1. Correct the bias of the MR brain data.

(a) Brain intensity normalization [86].

(b) GGMRF edge preservation [85].

2. Strip the skull using BET [70].

3. Estimate the LCDG models for brain and non-brain tissues using Step 2 results.

4. Form an initial $\mathrm{m}$ by voxel-wise classification using LCDG models found in Step 3.

5. Estimate analytically the Gibbs potentials for the pair-wise MGRF model of $m$ to identify the MGRF probability.

6. Calculate the distance map inside the binary mask obtained from BET using FMLS [88].

7. Generate a set of $N$ iso-surfaces (figure 22 (e)) using the distance map calculated in Step 6.

8. while $j \leq N$

(a) Select the $j^{\text {th }}$ iso-surfacer and classify its voxels using a Bayes classifier combining the first and second-order visual appearance features.

(b) Are all the voxels on the selected iso-surfaces classified only as brain tissue?

- No $\longrightarrow$ Go to Step 8 (a).

- Yes $\longrightarrow$ Break

9. Apply connected component component analysis to obtain the final results. 


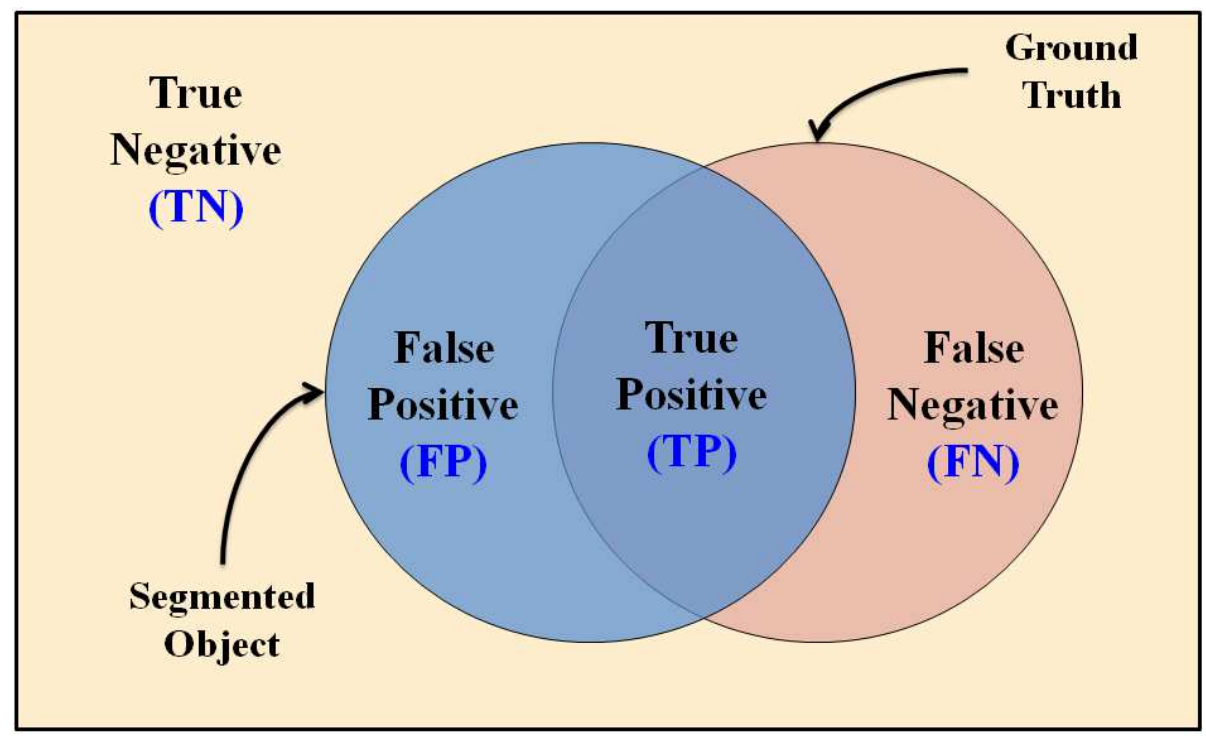

Figure 25. Segmentation errors calculation between the segmented and ground truth objects for the determination of the Dice similarity coefficient (D).

\section{Modified Hausdorff Distance $\left(\mathrm{H}_{95}\right)$}

The modified hausdorff distance $\left(\mathrm{H}_{95}\right)$ is used to measure the error distance between the segmented and ground truth objects. The Hausdorff distance (HD) [93] from a set $A_{1}$ to a set $A_{2}$ is defined as the maximum distance of the set $A_{1}$ to the nearest point in the set $A_{2}$ (as shown in figure 26):

$$
H\left(A_{1}, A_{1}\right)=\max _{c \in A_{1}}\left\{\min _{e \in A_{2}}\{d(c, e)\}\right\}
$$

where $c$ and $e$ denote points of set $A_{1}$ and $A_{2}$ respectively, and $d(c, e)$ is the Euclidean distance between $c$ and $e$.

The bidirectional HD between the segmented region $S R$ and its ground truth $G T$ is defined as:

$$
H_{B i}(G T, S R)=\max \{H(G T, S R), H(S R, G T)\}
$$

The 95th-perecntile bidirectional HD is used as a metric that measures the 


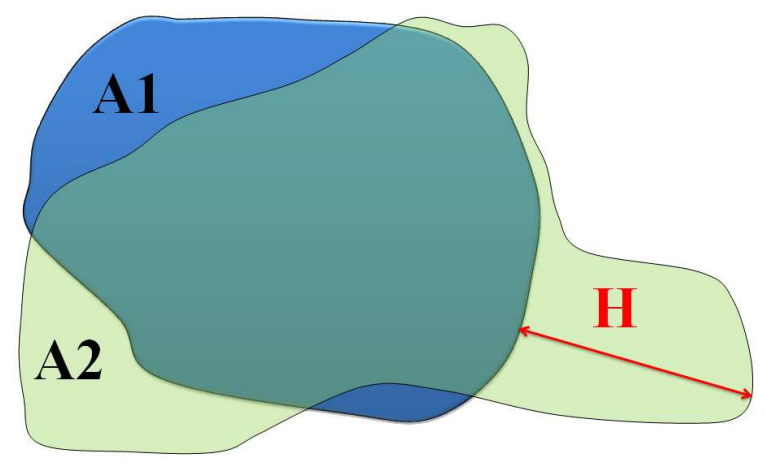

Figure 26. A schematic illustration for the Haussdorf distance (HD) calculation. segmentation accuracy, which is also known as the modified Hausdorrf Distance $\left(\mathrm{H}_{95}\right)$.

\section{Absolute Volume Difference (AVD)}

In addition to the $\mathrm{D}$ and the $\mathrm{H}_{95}$, the absolute volume difference (AVD) have been used as a third metric for measuring the segmentation accuracy. The AVD is the percentage volume difference between the segmentation and the ground truth as seen in figure 27 .

\section{Experimental Results}

In order to assess the robustness and performance of the proposed framework, it has been applied to 300 T1-weighted MR infant brain data sets which were obtained from the Infant Brain Imaging Study (IBIS) [94], and evaluated its accuracy using 9 data sets with known manually segmented ground truth that were obtained by an MR expert. MR data was acquired at 3T and consists of T1- and T2-weighted MR images of infants scanned at approximately 5-9 months old with voxel size of $1 \times 1 \times 1 \mathrm{~mm}^{3}$.

A step-wise brain extraction using the proposed approach for a selected axial cross-section of one subject is demonstrated in figure 22. The input MR image 
Segmented Volume

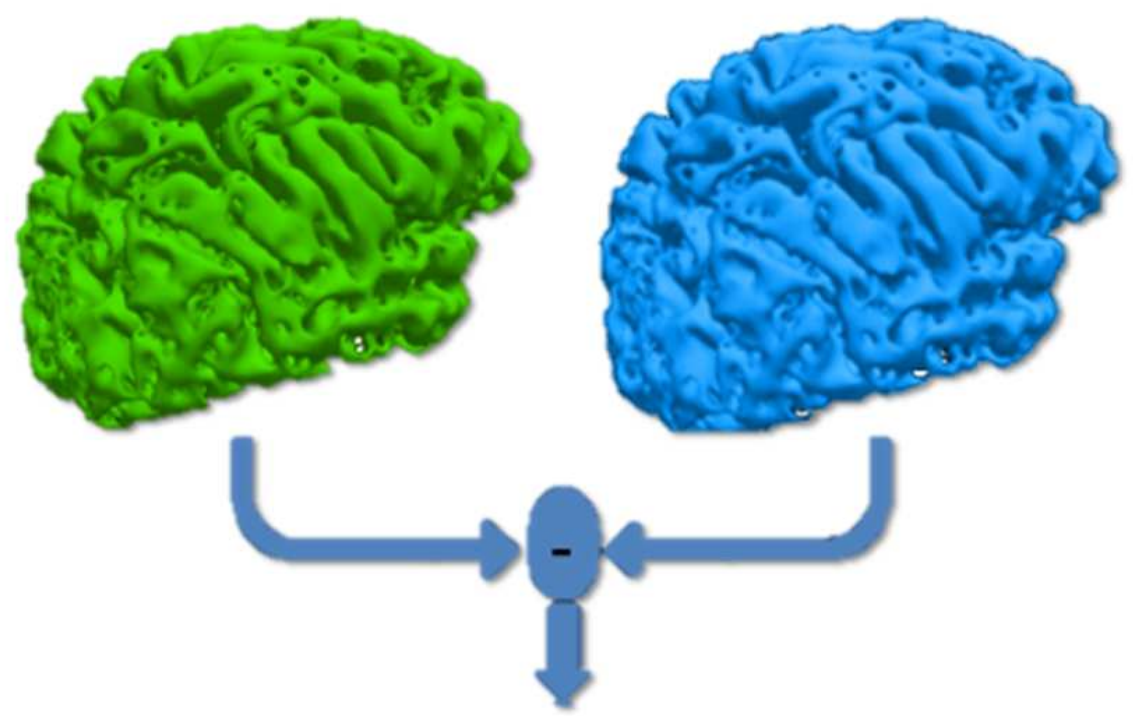

Ground Truth

AVD

Figure 27. A 3D schematic illustration for the absolute volume difference (AVD) estimation.

(figure 22(a)) is first bias corrected (intensity normalized Fig. 22 (b)) and applied to the 3D GGMRF [85] edge preservation (figure 22 (c)). This is followed by an initial brain extraction using BET [70] (figure $22(\mathrm{~d})$ ). Then, the proposed iso-surfaces based approach is employed to achieve the final segmentation as shown in figure 22 (f). It is clear from the results in figure 22 that the proposed framework provides more accurate infant brain extraction than BET. More segmentation results for different cross-sections from different subjects are shown in figure 28.

To highlight the advantage of the proposed framework, its performance has been compared to four widely-used brain extraction tools: the infant brain extraction and analysis toolbox (iBEAT) [95], the brain surface extractor (BSE) [82], the brain extraction tool (BET) [70], and the BET2 [96]. The comparative accuracy of the proposed approach versus the iBEAT, BSE, BET, and BET2 techniques on 

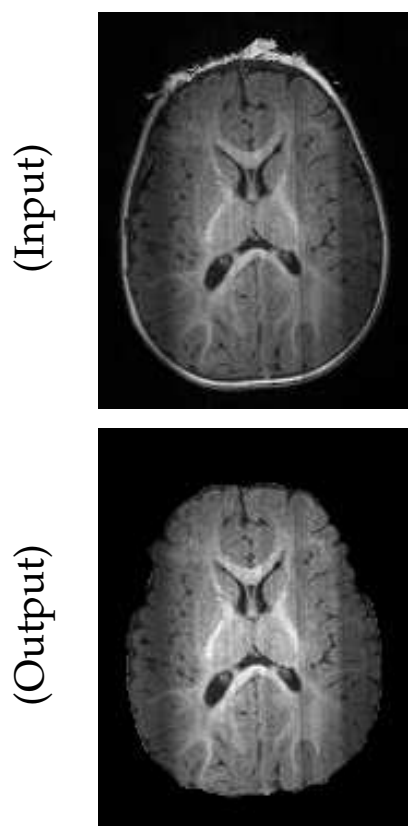

(a)
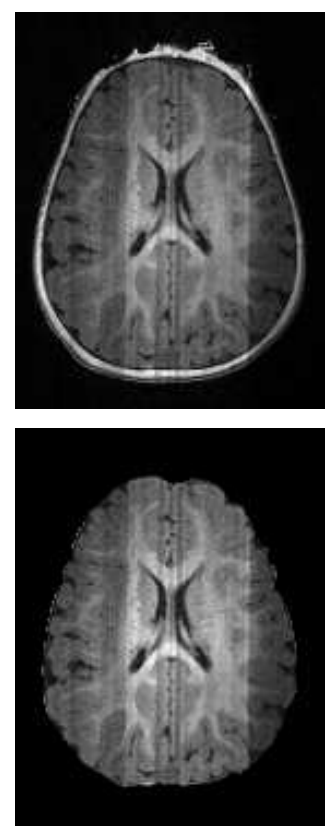

(b)
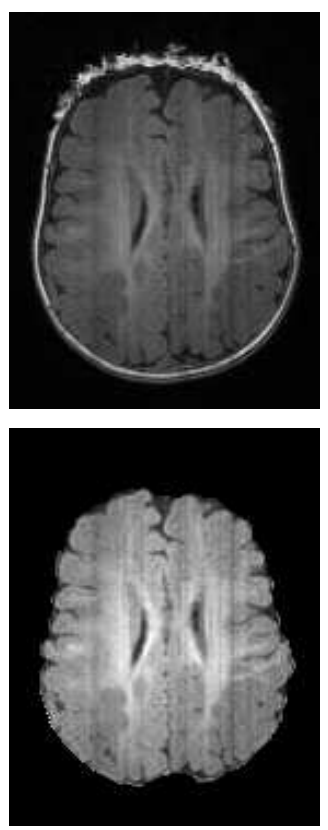

(c)
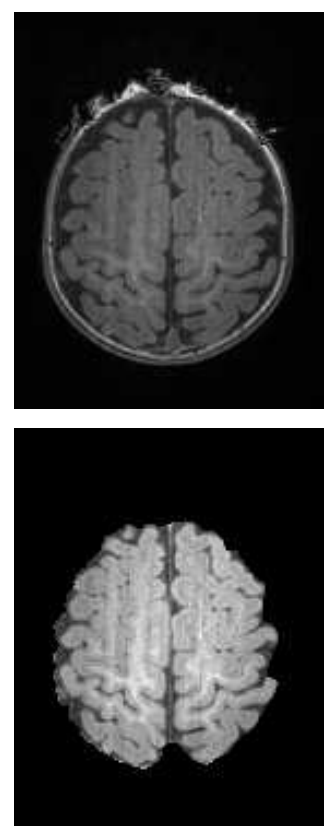

(d)

Figure 28. More segmentation results for different cross-sections from different subjects showing reliable brain extraction using the proposed approach.

representative images for 3 subjects is shown in figure 29. As demonstrated in figure 29 and the 3D extracted brains shown in figure 30, the proposed approach extracted the brain tissue more accurately compared with the other approaches. The lower performance of the BET [70] could be caused by its sensitivity to image noise and inhomogeneity, because this method relies only on voxels' intensity changes and does not account for spatial voxel interactions. On the other hand, the BET2 approach [70] slightly improves the brain extraction accuracy compared with the BET. However, unlike the BET, the BET2 requires both T1-and T2-weighted MR images and aligned to each other, which is not always applicable. Moreover, BSE [82] succeeds in accurately removing the skull but it removes small parts from the brain tissues as well, which may lead to inaccurate results for some clinical application (e.g. cortical thickness under-estimation). The iBEAT performs multiple complementary brain extractions by using a meta-algorithm, including BET and BSE. However, Table 1 shows that the proposed approach performs notably 

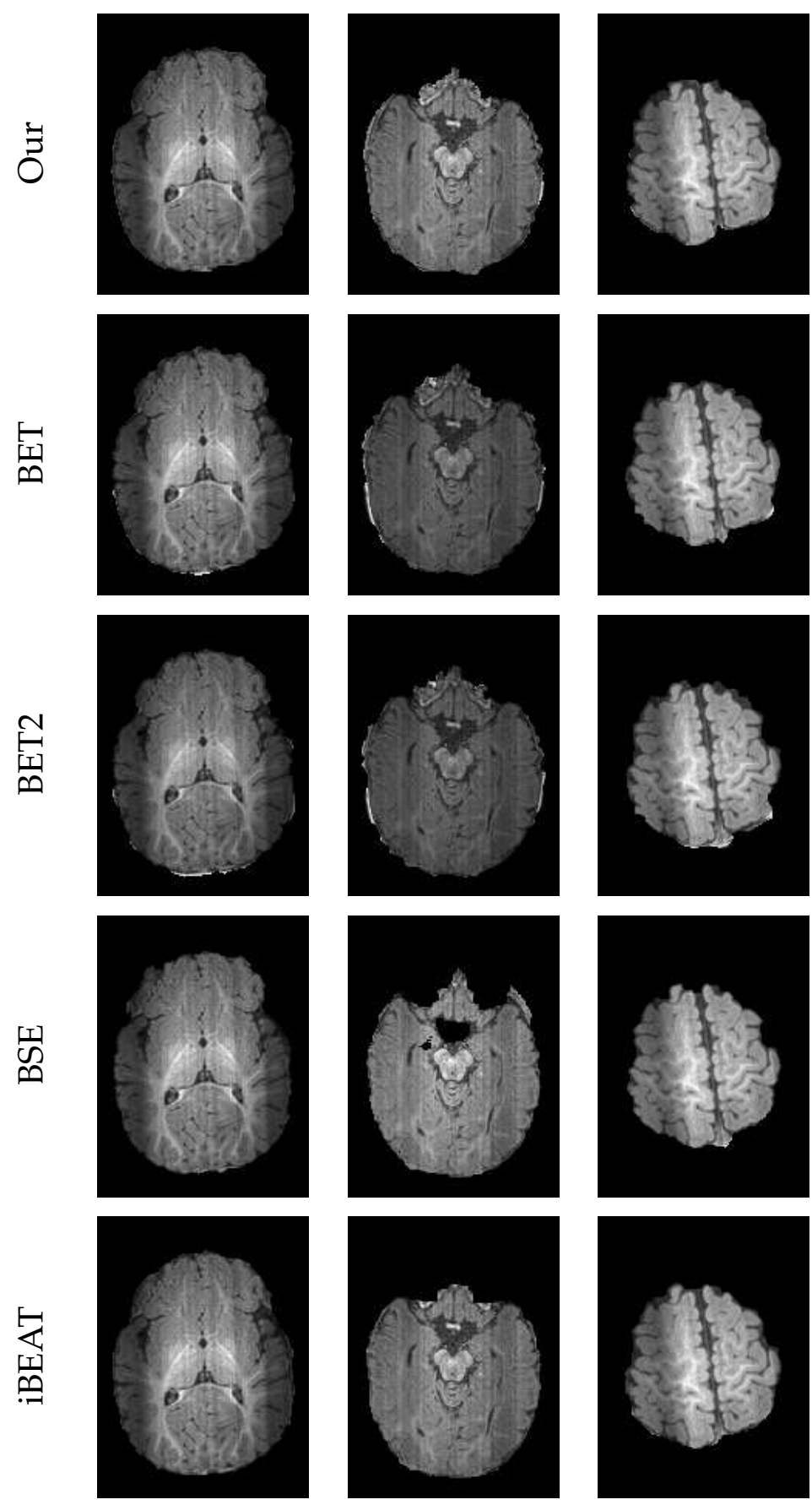

Subject \#1

Subject \#2

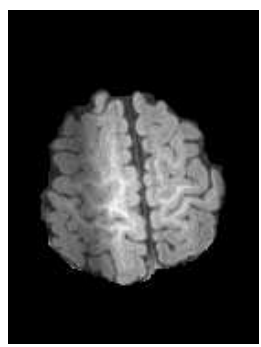

Subject \#3

Figure 29. Comparative brain extraction results for three independent subjects using the proposed approach, BET [70], BET2 [96], BSE [82], and iBEAT [95]. 
TABLE 1. Comparative accuracy of the proposed approach vs. the methods in [70, $82,95,96$ ] by the $\mathrm{D}, \mathrm{H}_{95}$, and AVD on 9 data sets with available ground truth ("SD "- standard deviation).

\begin{tabular}{|l|c|c||r|r||r|r|}
\cline { 2 - 7 } \multicolumn{1}{c|}{} & \multicolumn{6}{c||}{ Evaluation Metric } \\
\cline { 2 - 7 } \multicolumn{1}{c|}{} & \multicolumn{2}{c|}{$\mathrm{D}(\%)$} & \multicolumn{2}{c||}{$\mathrm{H}_{95}(\mathrm{~mm})$} & \multicolumn{2}{c|}{ AVD $(\%)$} \\
\hline Method & Mean $\pm \mathrm{SD}$ & $p$-value & Mean $\pm \mathrm{SD}$ & $p$-value & Mean $\pm \mathrm{SD}$ & $p$-value \\
\hline OUR & $95.86 \pm 0.77$ & - & $6.32 \pm 2.26$ & - & $3.76 \pm 2.52$ & - \\
iBEAT [95] & $94.30 \pm 2.47$ & 0.0448 & $9.95 \pm 4.98$ & 0.0179 & $6.84 \pm 5.25$ & 0.0292 \\
BSE [82] & $93.17 \pm 1.44$ & 0.0001 & $13.13 \pm 5.75$ & 0.0007 & $5.64 \pm 2.11$ & 0.0118 \\
BET2 [96] & $91.83 \pm 3.63$ & 0.0049 & $13.80 \pm 6.74$ & 0.0075 & $10.78 \pm 8.73$ & 0.0137 \\
BET [70] & $91.80 \pm 3.42$ & 0.0035 & $14.13 \pm 7.20$ & 0.0099 & $10.58 \pm 8.33$ & 0.0119 \\
\hline
\end{tabular}

better, according to the higher $\mathrm{D}$ and AVD values and lower $\mathrm{H}_{95}$ values. Table 1 compares this approach with the iBEAT, BSE, BET, and BET2, based on the D, $\mathrm{H}_{95}$, and AVD metrics. All metrics were obtained by comparing brain extraction results against the 9 data sets with available ground truth segmentation. As demonstrated in Table 1, the mean $\mathrm{D}, \mathrm{H}_{95}$, and AVD values for the proposed approach are $95.86 \pm 0.77 \%, 6.32 \pm 2.26 \mathrm{~mm}$, and $3.76 \pm 2.52 \%$, respectively, which confirm the high accuracy of this proposed approach. Statistical significance of the better performance of this approach with respect to other methods is confirmed by the paired $t$-tests ( $p$-values are less than 0.05).

\section{E Summary}

This Chapter has introduced a novel framework for the automated extraction of the brain from 3D infant MR images. The experimental results have shown that the fusion of stochastic and geometric models of the brain MRI data leads to more accurate brain extraction, when compared with widely-used brain extraction tools: iBEAT, BSE, BET, and BET2. These results were evaluated using the Dice 

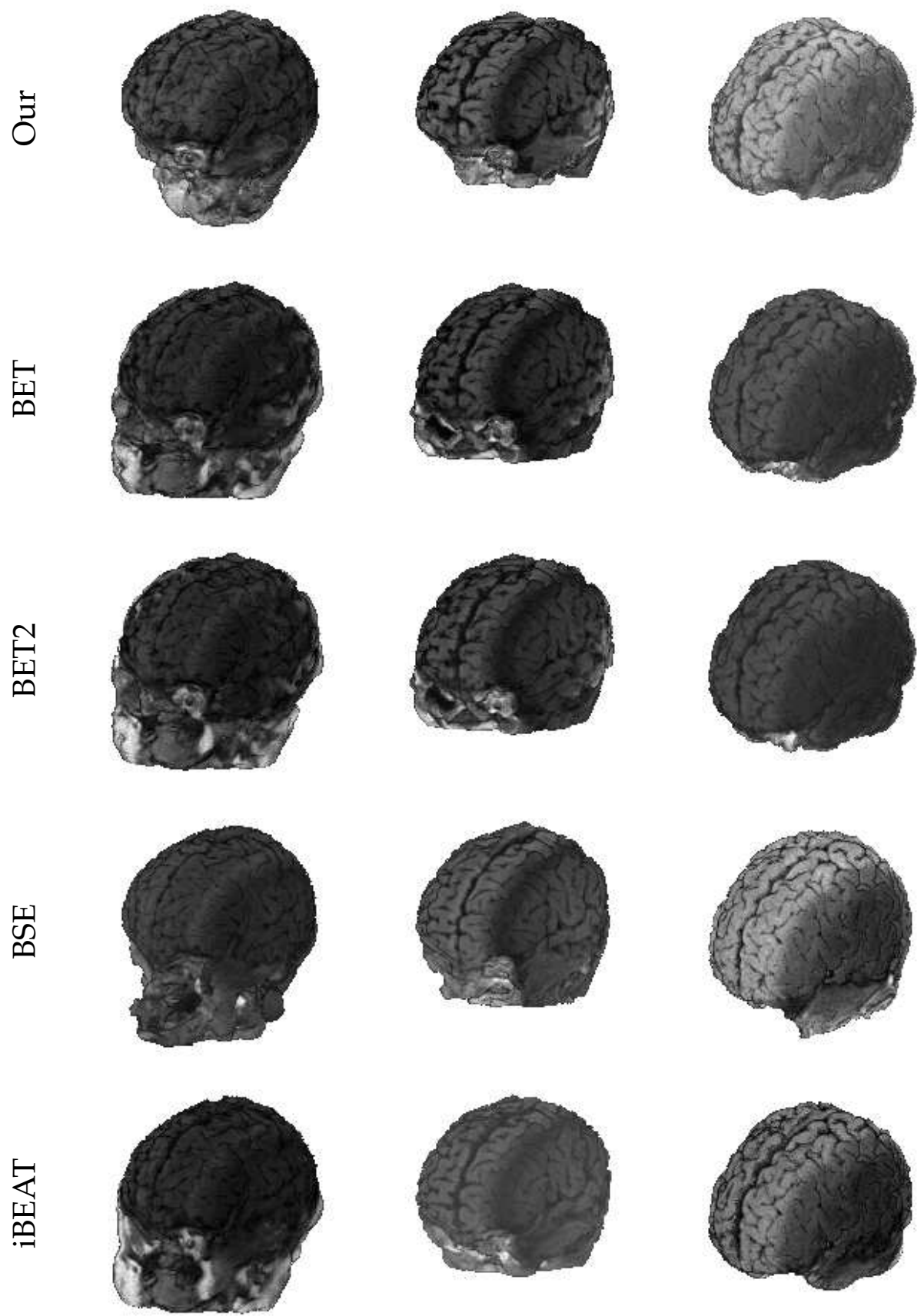

Subject \#1 Subject \#2

Subject \#3

Figure 30. 3D visualization of the segmented brain tissue for 3 different subjects using the proposed approach, BET [70], BET2 [96], BSE [82], and iBEAT [95]. 
similarity coefficient (D), 95-percentile modified Hausdorff distance $\left(\mathrm{H}_{95}\right)$, and the absolute volume difference (AVD) on 9 infant MR brain data sets. The next step after brain extraction is the brain tissue segmentation. Chapter III will elaborate the segmentation of the different brain structures for both adult and infant MRI, e.g. WM, GM, CSF, and other brain tissues. 


\section{CHAPTER III}

\section{BRAIN TISSUE SEGMENTATION}

Since the main focus of this thesis is the accurate classification of the infant brain tissue from structural magnetic resonance imaging (MRI). After the brain extraction and skull stripping step in Chapter II, this Chapter introduces a new framework for the segmentation of different brain structures from 3D MR brain images. The proposed segmentation framework is based on a shape prior built using a subset of co-aligned training images that is adapted during the segmentation process based on first- and second-order visual appearance characteristics of infant MRIs. These characteristics are described using voxel-wise image intensities and their spatial interaction features. To more accurately model the empirical grey level distribution of the brain signals, a linear combination of discrete Gaussians (LCDG) model having positive and negative components have been used. To accurately account for the large inhomogeneity in MR brain images, a higherorder Markov-Gibbs Random Field (MGRF) spatial interaction model that integrates higher-order cliques (third- and fourth-order) with the traditional secondorder cliques is proposed. The proposed approach was tested and evaluated on both in-vivo adult and infant 3D MR brain scans using three metrics: the Dice coefficient, the 95-percentile modified Hausdorff distance, and the absolute brain volume difference. The experimental results promise more accurate segmentation of MR brain images compared to the current widely-used segmentation tools. 


\section{A Introduction}

Accurate segmentation of brain tissues from magnetic resonance imaging (MRI) is an essential step in clinical diagnostics, therapy evaluation, human brain mapping, and neuroscience [67]. In particular, segmenting an infant brain MR image contributes much to the analysis and treatment of brain injury and disorder resulting from the infant brain prematurity [68]. However, MR brain tissue segmentation meets with challenges stemming from image noise, inhomogeneities, artifacts, such as e.g. partial volume effect, and discontinuities of boundaries due to similar visual appearance of adjacent brain structures. This thesis targets the infant brain MRI segmentation, which is more complicated than the adult brain tissue segmentation that may be based only on the image intensity. The intensitybased segmentation methods rely on the contrast between different types of tissues and high signal-to-noise ratios. Thus it is hindered by reduced contrast, higher noise from infants [66], and inverse contrast between the White Matter (WM) and Gray Matter (GM) in the infant brain MRIs [69], see figure 31.

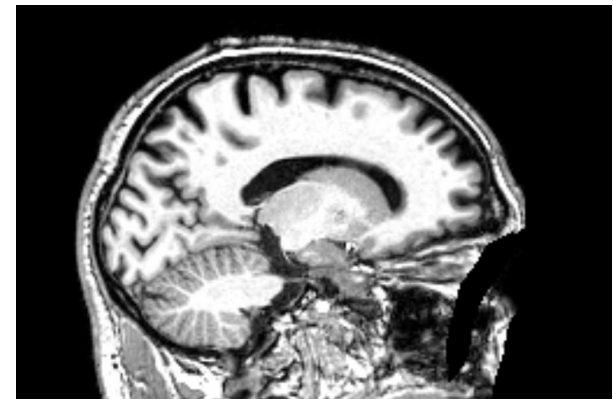

(a)

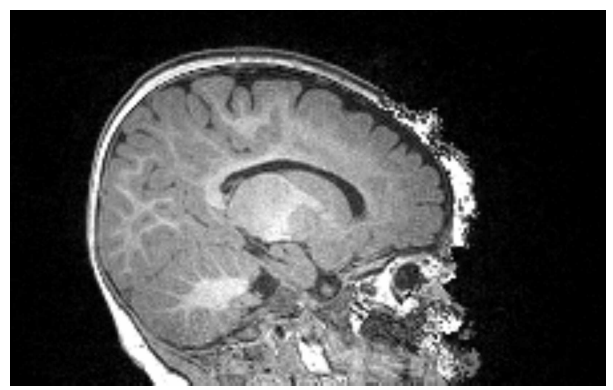

(b)

Figure 31. T1-weighted MRI for adult (a) and infant (b) brains.

Lower contrast between infant brain tissue classes stems from the fact that most of the WM is unmyelinated yet and its water content is close to the GM one. Moreover, both the WM and GM have the same intensity at about nine months 
of age [97]; hence, it is difficult to classify the brain tissues using only the intensity. Additionally, major partial volume effects occur due to the contrast inversion between the WM and GM comparing to the adult brain MRI. The unmyelinated WM intensity is just between intensities for the GM and Cerebrospinal Fluid (CSF) tissues, so that partial volume averaging often misclassifies the average between the latter two tissues as the unmyelinated WM [69]. Furthermore, the MRI factors, such as long scan duration, small voxel size, and low signal-to-noise ratio (SNR), also hinder the infants brain imaging. High-resolution images are essential to show the infant brain structure because of its much smaller size compared to then adult brains [68]. But small-size voxels lead to noisy infant MRI with reduced contrast, i.e. with low contrast-to-noise ratio (CNR). To improve the CNR, the scan duration has to be increased; however, it is too risky for an infant to be anesthetized for a long period. As it is important to minimize scan times, usually infants are being fed and then wrapped to prevent movement [98]. A large variety of segmentation techniques have been developed for the last two decades in order to address the brain MRI segmentation challenges. These techniques can be roughly classified into three main categories: (i) probabilistic, or statistical methods, (ii) atlas-based methods, and (iii) techniques based on deformable models.

\section{Probabilistic segmentation}

These algorithms involve prior models that describe the signal distributions of each brain structure. $\mathrm{Ng}$ et al. [99] segment MR brain images using the unsupervised $K$-means clustering of signals and an improved watershed algorithm. Signal clustering to produce an initial image segmentation before applying the improved watershed transform helped to overcome basic weaknesses of the latter, such as over-segmentation and sensitivity to false edges. A similar approach by Xue et al. [69] employed a parametric Gaussian density estimation with an ExpectationMaximization (EM) algorithm and constrained spatial homogeneity of the MR im- 
ages with a Markov random field (MRF) prior. Partial volume averaging effects have been eliminated by predicting the misclassification (e.g., of an "averaged" CSF and GM into an intensity similar to WM). Song et al. [100] proposed a probabilistic neural network (PNN) for segmenting the brain MRI. Probability density functions of the brain tissues were estimated from reference vectors generated by a self-organizing map (SOM). To reduce the partial volume averaging effects, weighting factors were added to the summation layer's patterns in a weighted probabilistic neural network (WPNN) and soft labeling was performed by a supervised Bayesian classifier. Automated segmentation of brain structures, such as WM, CSF, Central GM (CEGM), and Cortical GM (COGM) by Anbeek et al. [101] used T2-weighted and inversion recovery (IR) MRI of the neonatal brains. Probability maps to segment each brain tissue class with a $K$-nearest neighbor (KNN) classifier using voxel intensities and coordinates as features were constructed manually. A multi-label segmentation process combined the obtained classes. An automated MRI brain segmentation by Mayer et al. [102] combined spatial and intensity features into a high-dimensional feature space. An adaptive mean-shift classifier extracted a set of convergence modes, i.e. high-density points of a feature space, being good candidates for intensity-based classification. Brain tissues were classified by an intensity-based mode clustering. This approach was very effective with non-convex clustering. Fang et al. [103] developed a tree metrics (TM) based graph cut algorithm to segment the MRI brain tissues. After a brain MR image is classified using the TM, the goal labeling is inferred by "tree-cutting". In contrast to most of conventional iterative methods like the EM-based ones, which produce only locally optimal labeling, this algorithm needs no more than one sweep to generate the globally optimal labeling with respect to the TM. An automated segmentation by Ortiz et al. [104] classified the brain tissue with no prior information. The segmentation consists of feature extraction and classification. Extracted first order (pixel/voxel-wise), second order (pair-wise), moment, and scale-invariant 
features are classified by growing hierarchical SOMs (GHSOM). Wang et al. [105] segmented T1, T2 and diffusion-weighted brain images using a sparse representation of the complementary tissue distribution. Initially, the brain tissue is segmented into different structures using a patch-based technique with a library of multi-modality images, having been aligned with their ground-truth segmentation maps. Then the segmentation is refined by integrating geometric constraints.

Statistical-based techniques are fast to implement compared to other segmentation methods. However, they depend only on predefined probability models that cannot fit all of the possible real data distributions. This is due to the fact that actual intensity distributions of brain structures are greatly affected by several factors, such as the unique patient and scanner along with scanning parameters. Also, due to the similar intensities (gray levels) for the different brain tissue structures of the infant MR brain images, segmentation techniques only based on the intensity remain inaccurate.

\section{Atlas-based segmentation}

Atlas-based approaches have emerged as powerful segmentation tools. These approaches are based on shape priors about brain structures, and deal with the segmentation problem as a registration task. Ashburner et al. [106] introduced a generative framework that combined image registration, tissue classification, and bias correction. Their framework incorporated a smooth intensity variation and nonlinear registration with tissue probability maps using mixture of Gaussians. Pohl et al. [107] introduced a Bayesian model for simultaneous segmentation and registration. Their framework tried to exploit complementary aspects of registration and segmentation problems. In order to account for different physiological (patient size and weight) and scanning (scanner type and data acquisition protocol) parameters, Han et al. [108] introduced an intensity re-normalization procedure to adjust the prior atlas intensity model to new input data to overcome the 
problems stemming from using training data acquired from a different scanner than what used for the test data. The re-normalization process updates the classconditional densities for each atlas structure by applying a multi-linear atlas-image registration and histogram matching. Artaechevarria et al. [109] proposed a generalized local weighting voting scheme in which the fusion weights are adapted for each voxel based on local estimation of the segmentation performance. The local weighting voting outperforms traditional global strategies that estimate a single value for the segmentation accuracy for the whole image. Sabuncu et al. [110] proposed an automated, label fusion segmentation technique. In order to capture greater inter-subject anatomical variability, each training data set was individually co-registered to the test data set. Then, a nonparametric probabilistic model was employed to fuse the training labels to compute the final segmentation. Morin et al. [111] presented an atlas-based segmentation framework using random walks that combined registration and labeling propagation steps. They used a generative model to provide pixel label probabilities to improve the segmentation for high-confidence labels. To match the target images with atlas images, they used the Affine-Scale Invariant Feature Transform (ASIFT) [112] and Speeded Up Robust Features (SURF) [113] registration techniques. In order to avoid segmentation errors produced by registration imperfection, Lötjönen et al. [114] introduced an optimized pipeline for multi-atlas brain MRI segmentation. They introduced two approaches that combine multi-atlas segmentation and intensity modeling based on using EM and graph cuts for optimization. First, they register all atlases to the target data and a majority voting is applied to predict the segmentation of the target image. Then, the segmentation was improved using the intensity modeling as a post-processing step. Lijn et al. [115] introduced a segmentation method based on the combination of spatial features and appearance models. They generated a spatial probability map that is obtained from multiple atlas-target image registrations, to implement the spatial model. The tissue appearance was modeled 
by a KNN classifier using Gaussian scale-space features. Then, a Bayesian framework was used to combine both spatial and appearance models and a graph-cut approach [116] was used for optimization. Ledig et al. [117] introduced a framework for labeling whole brain scans by incorporating a global and stationary MRF to ensure consistency of the neighborhood relations between structures with an a priori defined model. Atlas-based segmentation techniques show more accuracy with respect to statistical-based techniques. Nevertheless, they are still challenged by atlas selection, combination, and the associated heavy computation time. Another major drawback of atlas-based segmentation algorithms is their dependency on the selected features that will be used to link between the test subject and the prior (training) data used in the construction of the atlas. For example most of the current techniques use signal intensity to find the correspondence between the data to be segmented and the prior atlas. This may lead to inaccurate segmentation results as signal intensities (gray levels) vary due to many factors, such as age, patient and scanner.

\section{Deformable models-based segmentation}

In order to obtain continuous segmentation of brain structures, deformable boundaries have also been recognized as more accurate segmentation techniques of MR brain tissues. Angelini et al. [118] introduced a multi-phase level set framework for automated segmentation of brain MRIs. The segmentation of the brain tissues (WM, GM and CSF) was solely based on homogeneity (average grey level) measures. To avoid the need for any prior information and to speed up numerical calculation, a random seed for initialization of the deformable boundaries was used. Colliot et al. [119] proposed a deformable model-based approach that used spatial constraints, represented as fuzzy subsets of the 3D image space, as an external force to control the boundary evolution. To avoid manual selection of the model parameters, a training step was required to estimate the spatial constraints 
parameters. Miri et al. [120] introduced a topology-preserving deformable model framework for the segmentation of brain MRI. They employed photometric constraints to guide the deformable model deformations to iteratively reclassify the points located at the evolving boundaries. A deformable model approach for the segmentation of brain regions from MR images was proposed by Liu et al. [71]. The deformable contour was implicitly represented by a set of Wendland's radial basis functions (RBFs) and is evolved by iterative updates of the locations of the RBFs. The updates of the RBFs locations were controlled by an external force that considers the intensity contrast across boundaries. Huang et al. [121] introduced an automated, hybrid deformable model framework that integrated both image edge geometry and voxel statistics features to regularize the convergence of the deformable contour. Del Fresno et al. [122] described a hybrid method that combined region growing and deformable models for segmentation of different structures in head MRI and Computed Tomography CT scans. Their approach used a Region-Growing $(\mathrm{RG})$ algorithm to compute an approximation of the objects. This is followed by generating closed and oriented surface meshes to enclose the region of interest. To improve the segmentation of noisy images, local neighborhood features of each voxel of the region boundary was used. The deformable model method geometry were constructed using the RG-list of boundary voxels generating a hole-free surface mesh. To better detect the structures of interest, the user could select few seeds for RG initial segmentation. Wang et al. [123] proposed a multi-phase level set framework to segment brain MR images with intensity inhomogeneity. They modeled the local image intensities using Gaussian distributions with different means and variances. Then, a variational approach minimizes an energy function to compute the means and variances that will guide the contour evolution towards the target boundaries. Bourouis et al. [124] developed a level set framework for segmenting brain tissues. Their framework employed an image registration step and a classification step for the initialization of the deformable 
boundary. The boundary evolution was controlled by a speed function that accounts for both boundary- and region-based properties. Ciofolo et al. [125] developed an automated framework based on level sets for simultaneous segmentation of multiple structures from brain MRIs. The evolution of each level set was driven by a fuzzy decision system that combines three factors: intensity distribution of the $3 \mathrm{D}$ MR volume, the relative position of the evolving contours, and a priori knowledge provided by an anatomical atlas.

The main advantage of deformable model-based segmentation techniques is the ability to segment connected (non-scattered) objects more accurately than the other segmentation methods. However, the accuracy of this method is based on the accurate design of the guiding forces (statistical, geometric, etc.) in addition to the initialization of the model.

In summary, current segmentation techniques for infant brain MRIs suffer several drawbacks. While statistical-based techniques may be quickly implemented, they depend on predefined probability models that are not capable of fitting all possible real data distributions that arise from uniqueness in patients and variations in scanners and scanning parameters. Additionally, segmentation techniques based on the intensity remain inaccurate due to similar intensities between infant brain structures.

To overcome the aforementioned limitations, a hybrid framework is presented that is shown in figure 32 . This framework uses an adaptive probabilistic model for the shape and first-order visual appearance of the MRI data. These models are combined with a novel fourth-order Markov-Gibbs Random Field (MGRF) spatial interaction model. These adaptive probabilistic models increase the segmentation accuracy by accounting for large inhomogeneities in infant MRIs and to reduce the effects of noise. 

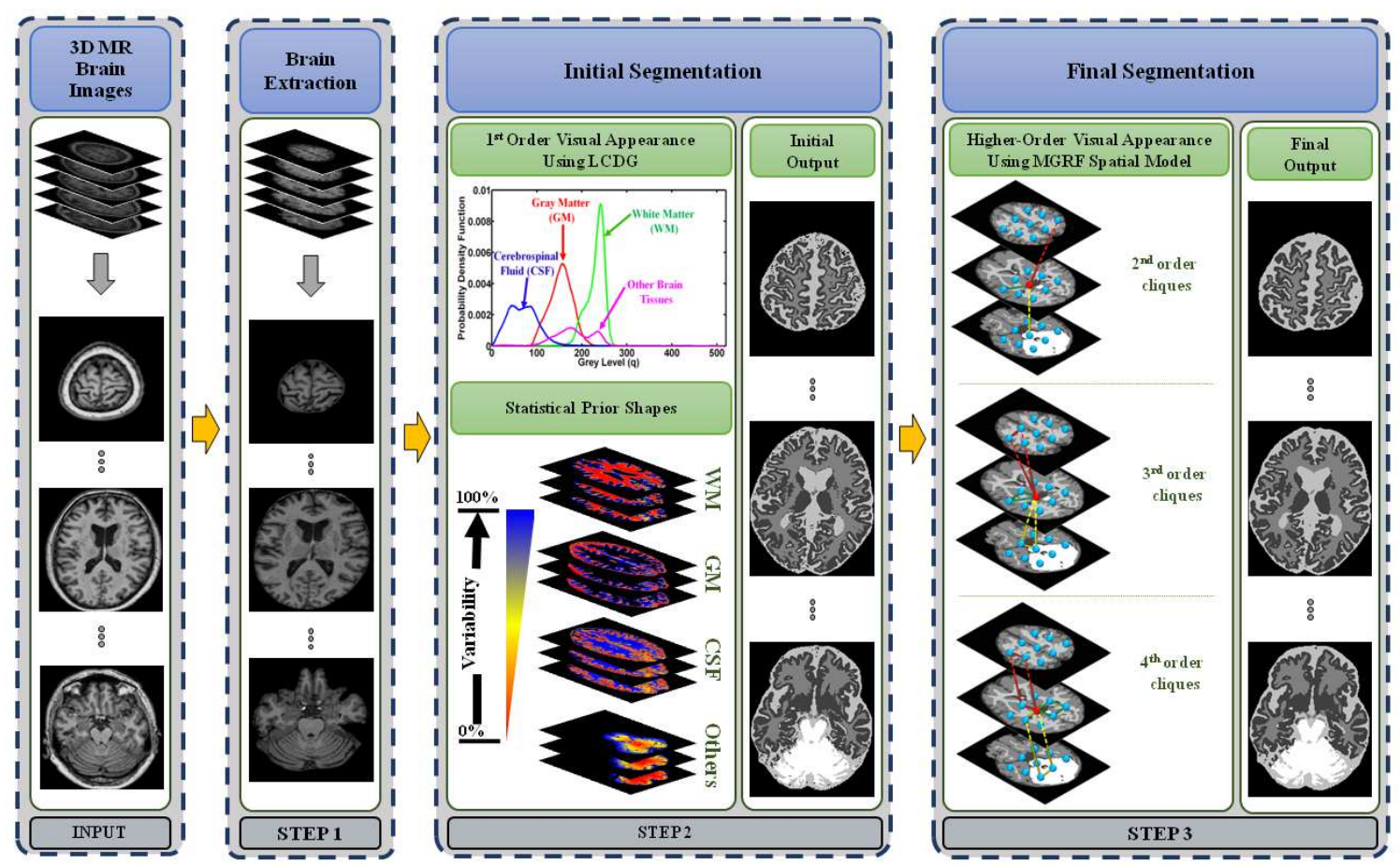

Figure 32. The basic steps of the proposed framework for brain structures segmentation from MR brain data.

\section{B Methods}

Let $\mathbf{R}=\{(x, y, z): 0 \leq x \leq X-1,0 \leq y \leq Y-1,0 \leq z \leq Z-1\} ;$ $\mathbf{Q}=\{0,1, \ldots, Q-1\}$; and $\mathbf{L}=\{0, \ldots, L\}$ denote a finite $3 \mathrm{D}$ arithmetic lattice of the size of $X Y Z$ supporting grayscale images and their region (segmentation) maps, a finite set of $Q$ integer gray values, and a set of region labels $L$, respectively. Let $\mathbf{g}=\left\{g_{x, y, z}:(x, y, z) \in \mathbf{R} ; g_{x, y, z} \in \mathbf{Q}\right\}$ and $\mathbf{m}=\left\{m_{x, y, z}:(x, y, z) \in \mathbf{R} ; m_{x, y, z} \in \mathbf{L}\right\}$ be a grayscale image taking values from $\mathbf{Q}$, i.e., $\mathbf{g}: \mathbf{R} \rightarrow \mathbf{Q}$, and a region map taking values from $\mathbf{L}$, i.e., $\mathbf{m}: \mathbf{R} \rightarrow \mathbf{L}$, respectively. An input brain image, $\mathbf{g}$, coaligned to the training data base, and its map, $\mathrm{m}$, are described with a joint probability model: $P(\mathbf{g}, \mathbf{m})=P(\mathbf{g} \mid \mathbf{m}) P(\mathbf{m})$, which combines a conditional distribution of the images given the map $P(\mathbf{g} \mid \mathbf{m})$, and an unconditional probability distribution of maps $P(\mathbf{m})=P_{\mathrm{sp}}(\mathbf{m}) P_{\mathbf{V}}(\mathbf{m})$. Here, $P_{\mathrm{sp}}(\mathbf{m})$ denotes a weighted shape prior, and $P_{\mathbf{V}}(\mathbf{m})$ is a Gibbs probability distribution with potentials $\mathbf{V}$, which specifies a 
MGRF model of spatially homogeneous maps $\mathbf{m}$. Details of the model's components are outlined in the next sections.

\section{First-Order Intensity Model}

The first-order visual appearance of each brain label is modeled by separating a mixed distribution of voxel intensities of the brain MRIs into individual components associated with the dominant modes of the mixture. The latter is precisely approximated with a Linear Combinations of Discrete Gaussians (LCDG) [126] with positive and negative components, which is based on a modified version of the classical Expectation-Maximization (EM) algorithm. The LCDG model is explained in detail in Chapter II.

Let $\boldsymbol{\Psi}_{\theta}=(\psi(q \mid \theta): q \in \mathbf{Q})$ denote a discrete Gaussian (DG) with parameters $\theta=(\mu, \sigma)$, integrating a continuous 1D Gaussian density with mean $\mu$ and variance $\sigma^{2}$ over successive gray level intervals. The LCDG with four dominant (WM, GM, CSF, and other brain tissues) positive DGs and $C_{\mathrm{p}} \geq 4$ positive and $C_{\mathrm{n}} \geq 0$ negative subordinate DGs is [126]:

$$
P_{\mathbf{w}, \boldsymbol{\Theta}}(q)=\sum_{k=1}^{C_{\mathrm{p}}} w_{\mathrm{p}: k} \psi\left(q \mid \theta_{\mathrm{p}: k}\right)-\sum_{\kappa=1}^{C_{\mathrm{n}}} w_{\mathrm{n}: \kappa} \psi\left(q \mid \theta_{\mathrm{n}: \kappa}\right)
$$

where all the weights $\mathbf{w}=\left[w_{\mathrm{p}: k}, w_{\mathrm{n}: k}\right]$ are non-negative and meet an obvious constraint $\sum_{k=1}^{C_{\mathrm{p}}} w_{\mathrm{p}: k}-\sum_{\kappa=1}^{C_{\mathrm{n}}} w_{\mathrm{n}: \kappa}=1$. All LCDG parameters, including the DGs numbers, are estimated from the mixed empirical distribution to be modeled using the modified EM algorithm [126].

\section{MGRF Model With Second- and Higher-order Cliques}

In addition to the first-order visual appearance model, the spatial interactions between the brain voxels are also taken into account. However, existing spatial models (e.g., [127]) account only for second-order cliques which can not ac- 
count for the large inhomogeneity of the brain MRIs especially for infants. Therefore, a higher-order MGRF spatial interaction model is used, which adds to the pairwise cliques the families of the triple and quad cliques (figure 33(b,c)) with analytical estimation of the potentials. The proposed approach has the ability to account for the large inhomogeneity of brain MR images, thus, reducing the noise effects and increasing the segmentation accuracy. Details of the proposed higherorder MGRF model are described below.

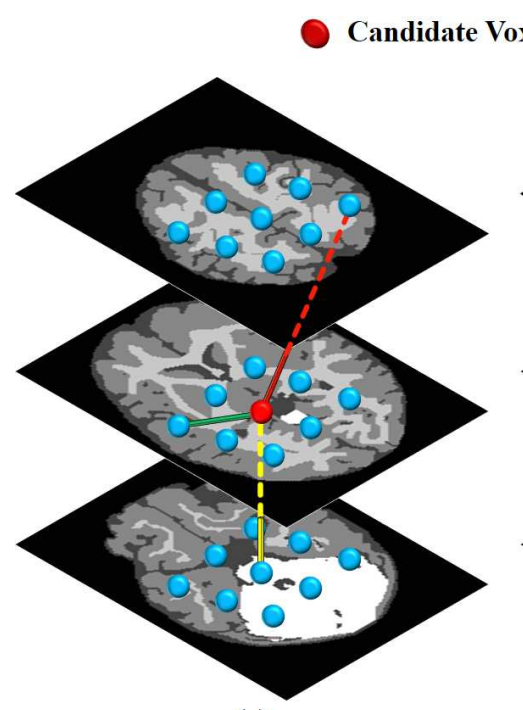

(a)

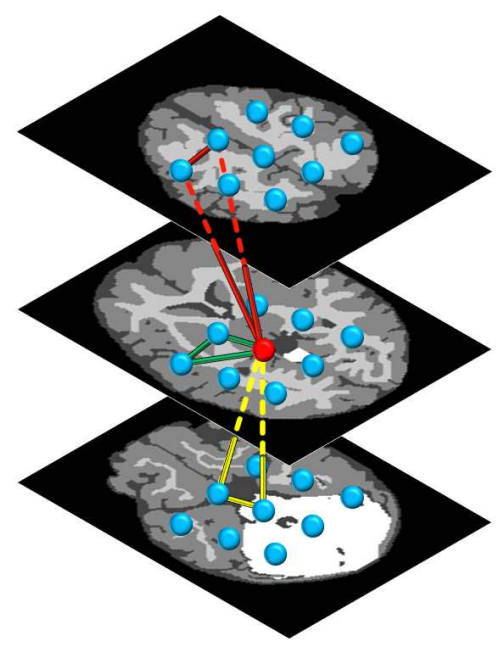

(b)
26-Nearest Neighbors

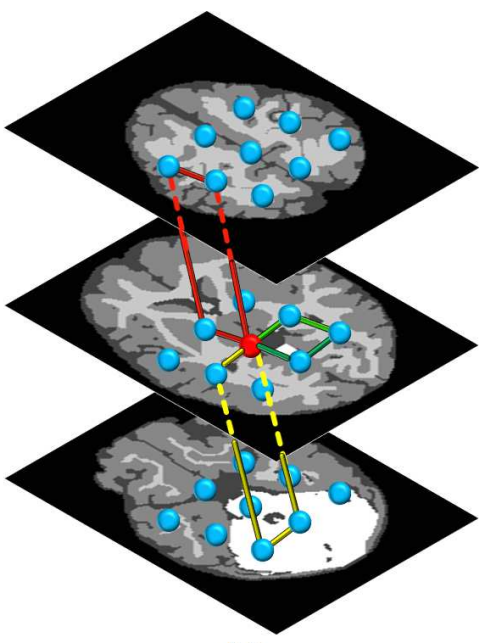

(c)

Figure 33. Samples of the second- $(a)$, third- $(b)$, and fourth-order (c) cliques for the 26-neighborhood (graph cliques are shown in different colors for visualization purpose).

Let $\mathbf{C}_{a}$ denote a family of $s$-order cliques of an interaction graph with nodes in the 3D lattice sites $(x, y, z)$ and edges connecting the interacting, or interdependent, sites (see figure 33). To account for large variations of the brain MRI scans, the label interactions are modeled by a spatially homogeneous MGRF with up to fourth-order interactions over the nearest 26-neighborhoods of voxels:

$$
P_{\mathbf{V}}(\mathbf{m})=\frac{1}{Z_{\mathbf{V}}} \exp \left(\sum_{a=1}^{A} \sum_{\mathbf{c} \in \mathbf{C}_{a}} V_{a}(\mathbf{m}(x, y, z):(x, y, z) \in \mathbf{c})\right)
$$


where $A$ clique families describe the geometry of the graph interactions, $\mathbf{V}=\left[V_{a}:\{0, \ldots, L\} \rightarrow(-\infty, \infty): a=1, \ldots, A\right]$ is a collection of Gibbs potential functions $V_{a}$ for the families $\mathrm{C}_{a}$, and the partition function $Z_{\mathbf{V}}$ normalizes the probabilities over the parent population $\mathbb{M}=\{0, \ldots, L\}^{X Y Z}$ of all maps. An initial region map $\mathbf{m}$, obtained by the voxel-wise classification, allows for analytically approximating the maximum likelihood estimates of the potentials and computing the voxel-wise probabilities of the region labels. For symmetry sake, only equality or inequality of the labels in clique $\mathbf{c}$ is taken into account. The second- third- and forth-order potentials are given by equations (12), (13), and (14), respectively:

$$
\begin{gathered}
V_{a}\left(m_{\mathbf{p}_{1}}, m_{\mathbf{p}_{2}}\right)=\left\{\begin{array}{cl}
V_{2: a: e q} & \text { if } m_{\mathbf{p}_{1}}=m_{\mathbf{p}_{2}} \\
-V_{2: a: e q} & \text { otherwise }
\end{array}\right. \\
V_{a}\left(m_{\mathbf{p}_{1}}, m_{\mathbf{p}_{2}}, m_{\mathbf{p}_{3}}\right)= \begin{cases}V_{3: a: \mathrm{eq}_{3}} & \text { if } m_{\mathbf{p}_{1}}=m_{\mathbf{p}_{2}}=m_{\mathbf{p}_{3}} \\
-V_{\text {::a:eq }} & \text { otherwise }\end{cases} \\
V_{a}\left(m_{\mathbf{p}_{1}}, m_{\mathbf{p}_{2}}, m_{\mathbf{p}_{3}}, m_{\mathbf{p}_{4}}\right)= \begin{cases}V_{4: a: \mathrm{eq}_{4}} & \text { if } 4 \text { equal labels } \\
V_{4: a: \mathrm{eq}_{3}} & \text { if } 3 \text { equal labels } \\
-\left(V_{4: a: \mathrm{eq}_{3}}+V_{4: a: \mathrm{eq}_{4}}\right) & \text { otherwise }\end{cases}
\end{gathered}
$$

where $m_{\mathbf{p}_{i}}$ is the region map label at the voxel $\mathbf{p}_{i}=\left(x_{i}, y_{i}, z_{i}\right)$. The proposed analytical approximation of the Gibbs potentials from a given map $\mathbf{m}$ extends earlier second-order MGRFs (e.g., [127]) to the higher-order models. The complete proof for the higher-order MGRF model is detailed in the supplementary materials.

\section{Adaptive Shape Model}

To enhance the segmentation accuracy, expected shapes of each brain label are constrained with an adaptive probabilistic shape prior. To create the shape 
database, a training set of images collected from different brain subjects, are coaligned by 3D affine transformations with 12 degrees of freedom ( 3 for the 3D translation, 3 for the 3D rotation, 3 for the 3D scaling, and 3 for the 3D shearing) in a way that maximizes their Mutual Information (MI) [128]. The shape prior is a spatially variant independent random field of region labels for the co-aligned data:

$$
P_{\mathrm{sp}}(\mathbf{m})=\prod_{(x, y, z) \in \mathrm{R}} p_{\mathrm{sp}: x, y, z}\left(m_{x, y, z}\right)
$$

where $p_{\mathrm{sp}: x, y, z}(l)$ is the voxel-wise empirical probabilities for each brain label $l \in \mathbf{L}$. For each input MR data to be segmented, the shape prior is constructed by an adaptive process guided by the visual appearance features of the input MRI data. First, the subject from the shape database that has the best match with the input subject (i.e., highest similarity) is selected using the normalized cross correlation similarity coefficient. The selected subject is then used as a reference prototype to co-align the input subject using the 3D affine transformation described above. Algorithm 2 has been used in order to estimate the shape prior probabilities for each voxel in the test subject. The complete segmentation framework steps are summarized in Algorithm 3.

\section{Experimental Results}

In order to highlight the accuracy and robustness of the presented segmentation approach, it has been tested on twenty thick T1-weighted adult MR brain images acquired at the UMC Utrecht, the Netherlands, from patients with diabetes and their matched controls (having an increased cardiovascular risk) with varying degrees of atrophy and white matter lesions (age $>50$ ). These twenty data sets were acquired with a 3T MRI scanner with voxel size $0.958 \mathrm{~mm} \times 0.958 \mathrm{~mm} \times$ $3.0 \mathrm{~mm}$, and are divided into fifteen subject for testing and five subjects provided with full manual segmentations of GM, WM and CSF for training. The proposed 
Algorithm 2 Key Steps for Creating the Adaptive Shape Model

1. Construct the shape database through a co-alignment of the biased corrected training brains (both grey scale and manually segmented).

2. Use normalized cross correlation to measure the similarity between the test subject and each subject in the shape database, and choose the database subject that has the highest similarity to act as the reference in the registration process.

3. Register the test subject to the selected reference subject using 3D affine transformations with 12 degrees of freedom that maximizes their MI [128].

4. For each voxel in the test subject, calculate its shape prior probability according to the following steps:

(a) Use the obtained transformation matrix (T) to transform each voxel to the shape database domain.

(b) Construct a 3D window with initial size of $N_{1 i} \times N_{2 i} \times N_{3 i}$.

(c) Search inside the window for voxels with corresponding grey level in all training data sets.

(d) If needed, increase the window size and redo the search until a nonempty result is found.

(e) Create the labels' probabilities based on the relative occurrence of each label from the search results.

brain tissue segmentation approach has been applied to classify T1-weighted MR brain images to the following four classes: WM, GM, CSF, and other brain tissues. Empirical gray level density for the T1-weighted MR brain images, after removing the brain skull, and its estimated four dominant densities are shown in figure 34 . Figure 35 demonstrates a step by step marginal density estimation for each class ( $1^{\text {st }}$-order visual appearance descriptor) using the LCDG model. The first step is to remove the skull using the proposed brain extraction in Algorithm 1 as shown in figure 36(b). The second step is to get the initial region map by using the estimated LCDG models for each class (see figure 35(f)) and the prior shapes as shown in figure 36(c). Finally, the initial map is refined by using the proposed three descriptors 
Algorithm 3 Key Steps for the Proposed Segmentation Approach

1. Use the developed automated approach in Algorithm 1 to remove the skull from T1-weighted MR brain images.

2. Approximate the marginal intensity distribution $P(\mathrm{~g})$ of the T1-weighted MR brain image using an LCDG with four dominant modes (WM, GM, CSF, and other brain tissues).

3. Form an initial region map $\mathbf{m}$ using the marginal estimated density and prior shape of each brain label.

4. Find the Gibbs potentials for the MGRF model (for both pair-wise and higher-order cliques) from the initial map $\mathbf{m}$.

5. Improve $\mathbf{m}$ using the voxel-wise Iterative Conditional Mode (ICM) algorithm [129].

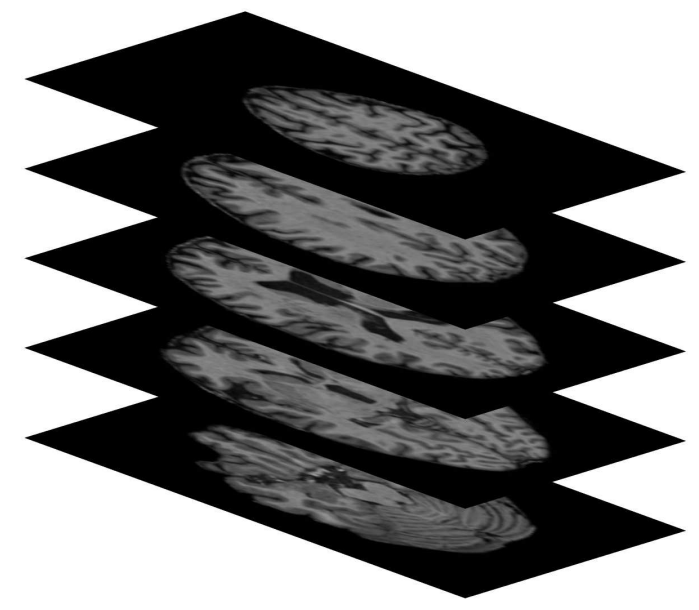

(a)

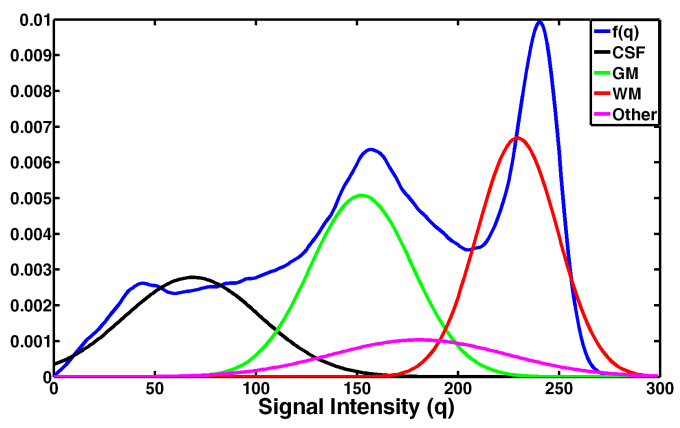

(b)

Figure 34. Typical T1-weighted MR brain images (a) and its normalized empirical density $f(q)(\mathbf{b})$.

( $1^{\text {st }}$-order visual appearance, 3D spatial MGRF, and prior shape models) to get the final segmentation as shown in figure 36(d). Figure 37 shows the 3D results of the proposed segmentation approach.

The performance of the proposed segmentation framework has been evaluated using three performance metrics: (i) the Dice similarity coefficient (D) [92], 


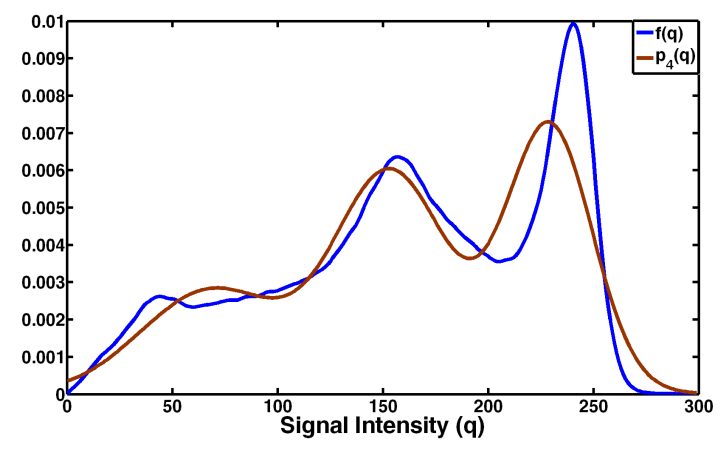

(a)

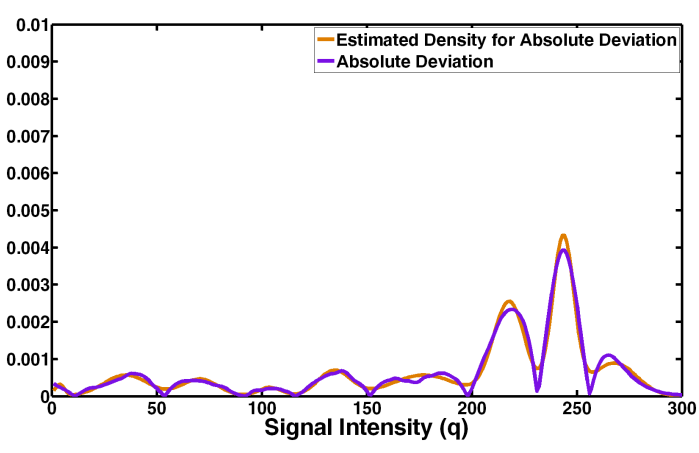

(c)

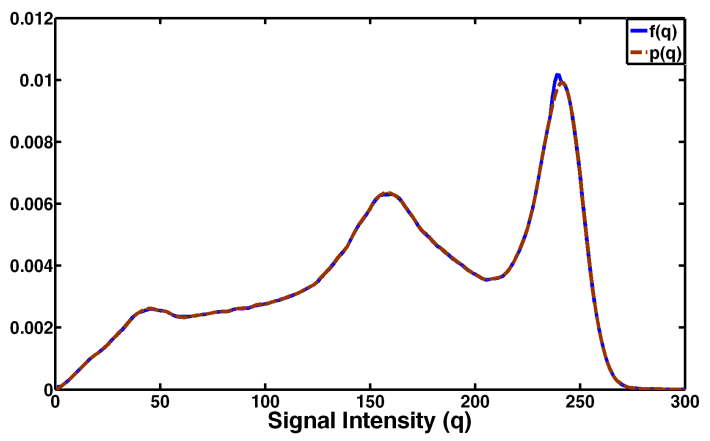

(e)

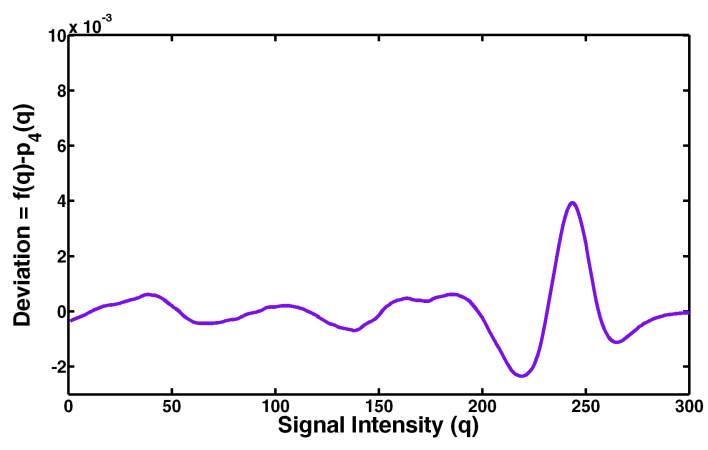

(b)

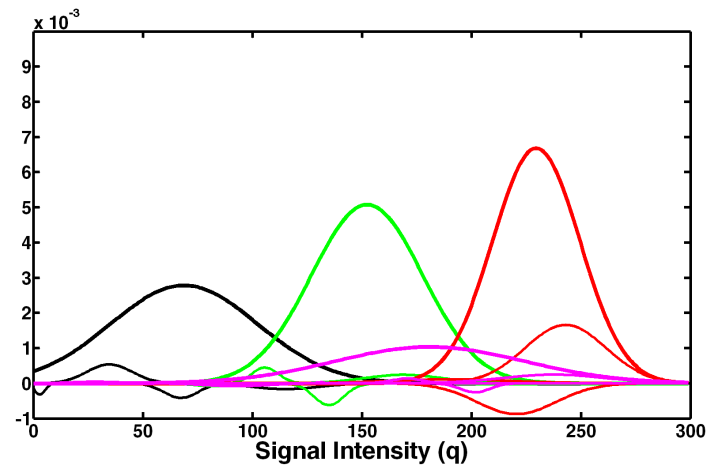

(d)

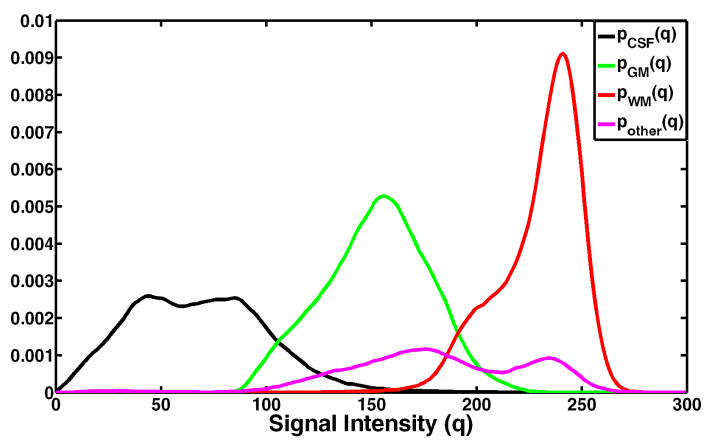

(f)

Figure 35. (a) Estimated density using only the four dominant Gaussian components $\left(p_{4}(q)\right)$, (b) deviation between $f(q)$ and $p_{4}(q)$, (c) estimated density of absolute deviation, (d) LCDG components, (e) final estimated density $(p(q))$, and (f) final estimated marginal density for each class. 

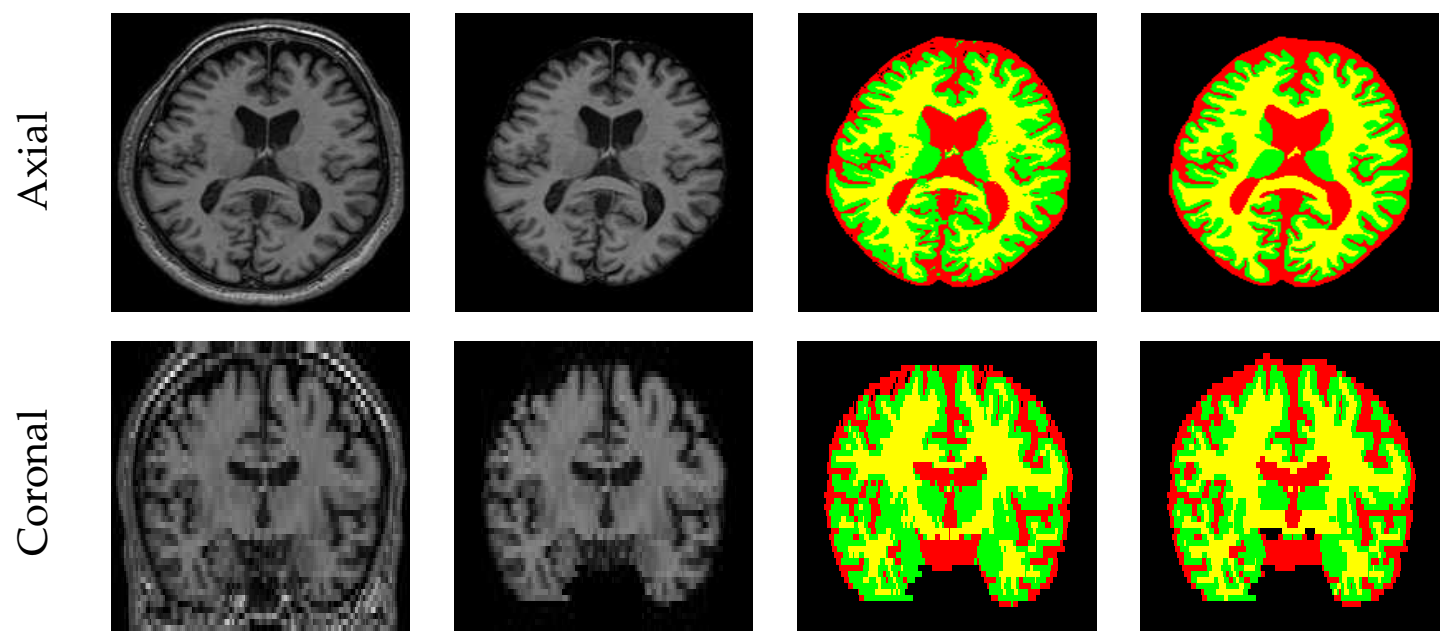

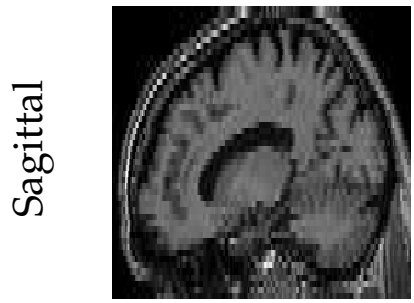

(a)

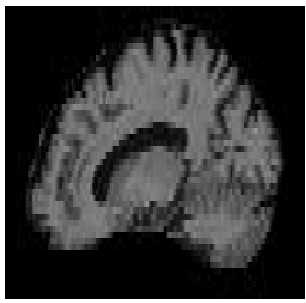

(b)

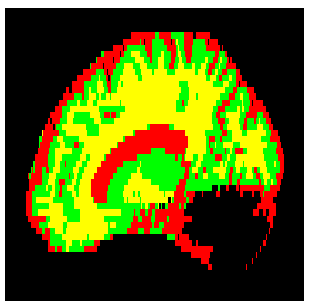

(c)

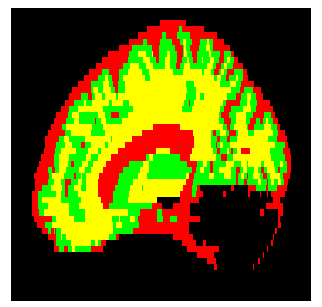

(d)

Figure 36. Segmentation results of the proposed segmentation approach. Segmentation performed in 3-dimensions; results projected onto 2D planes for visualization. (a) 2D profile of the original T1-weighted MR brain images, (b) MR brain data after removing the skull using BET software [70,96], (c) initial segmentation results using $1^{s t}$-order visual appearance and prior shape models, and (d) final segmentation results using the proposed three models. Note that WM, GM, and CSF are shown in yellow, green, and red colors, respectively.

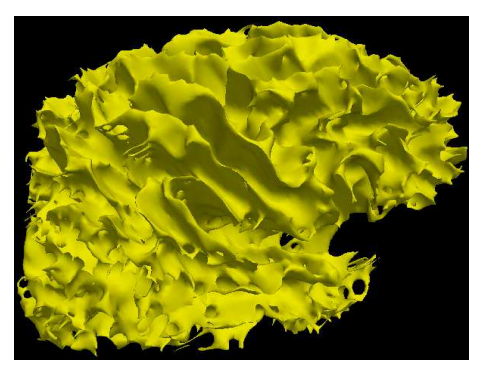

(a)

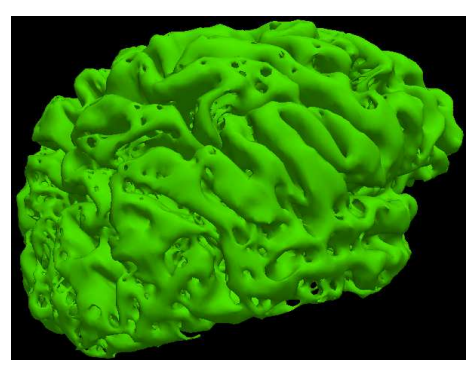

(b)

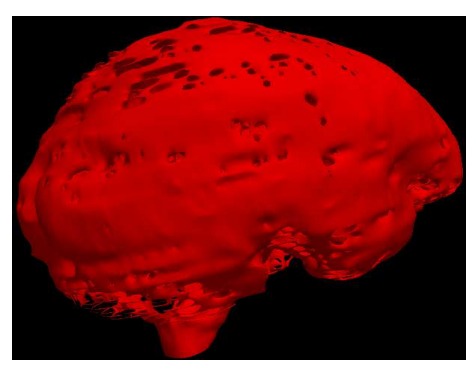

(c)

Figure 37. 3D visualization for the adult brain segmentation results: (a) WM, (b) GM, and (c) CSF. 
TABLE 2. The proposed brain tissue segmentation approach results for the fifteen test adult brains evaluated by MRBrainS13 [130].

\begin{tabular}{|c|c|c|c|c|c|c|c|c|c|c|c|c|c|c|c|}
\hline \multirow{2}{*}{ no. } & \multicolumn{3}{|c|}{ GM } & \multicolumn{3}{|c|}{ WM } & \multicolumn{3}{|c|}{ CSF } & \multicolumn{3}{|c|}{ Brain } & \multicolumn{3}{|c|}{ ICV } \\
\hline & $D$ & $H_{95}$ & $A V D$ & $D$ & $H_{95}$ & $A V D$ & D & $H_{95}$ & $A V D$ & D & $H_{95}$ & $A V D$ & D & $H_{95}$ & $A V D$ \\
\hline 1 & 85.91 & 1.36 & 7.04 & 89.26 & 1.92 & 6.84 & 77.69 & 3.00 & 2.67 & 96.00 & 1.92 & 1.43 & 97.31 & 3.56 & 1.73 \\
\hline 2 & 81.21 & 1.36 & 9.17 & 86.65 & 1.92 & 2.71 & 79.12 & 2.71 & 8.59 & 93.89 & 1.92 & 6.85 & 96.48 & 3.29 & 1.56 \\
\hline 3 & 82.25 & 1.92 & 4.08 & 85.75 & 3.00 & 14.9 & 78.18 & 2.71 & 0.52 & 94.50 & 1.92 & 3.23 & 96.71 & 3.03 & 2.06 \\
\hline 4 & 82.48 & 1.92 & 10.0 & 88.85 & 2.14 & 2.29 & 75.02 & 2.71 & 1.28 & 95.02 & 2.71 & 4.11 & 96.88 & 3.03 & 2.66 \\
\hline 5 & 84.57 & 1.92 & 11.6 & 85.91 & 1.92 & 10.8 & 81.52 & 2.14 & 8.64 & 95.33 & 2.71 & 3.54 & 97.20 & 3.15 & 0.03 \\
\hline 6 & 81.36 & 1.36 & 8.71 & 88.52 & 1.92 & 5.10 & 82.42 & 1.92 & 6.87 & 93.60 & 1.92 & 7.15 & 97.48 & 2.87 & 1.78 \\
\hline 7 & 83.16 & 1.92 & 15.1 & 86.34 & 2.14 & 20.0 & 82.16 & 2.14 & 3.93 & 95.32 & 2.71 & 1.77 & 97.57 & 3.15 & 0.07 \\
\hline 8 & 84.11 & 1.36 & 20.3 & 88.28 & 1.92 & 15.5 & 82.80 & 2.71 & 17.0 & 95.10 & 2.71 & 6.95 & 97.11 & 3.15 & 0.71 \\
\hline 9 & 84.03 & 1.36 & 5.93 & 91.57 & 1.36 & 3.12 & 77.42 & 3.29 & 37.4 & 95.30 & 2.87 & 4.57 & 95.57 & 3.83 & 7.35 \\
\hline 10 & 82.54 & 1.92 & 16.9 & 86.80 & 2.88 & 20.2 & 68.22 & 3.46 & 27.0 & 94.82 & 3.15 & 2.99 & 95.41 & 3.95 & 3.91 \\
\hline 11 & 79.77 & 1.92 & 0.23 & 87.71 & 3.03 & 12.8 & 81.71 & 1.92 & 11.2 & 94.56 & 1.92 & 5.85 & 97.48 & 2.87 & 0.06 \\
\hline 12 & 83.28 & 1.36 & 6.28 & 90.18 & 1.36 & 2.01 & 83.86 & 2.14 & 8.18 & 94.97 & 2.14 & 4.35 & 97.59 & 2.71 & 0.18 \\
\hline 13 & 82.87 & 1.92 & 8.13 & 90.39 & 1.92 & 9.14 & 73.25 & 3.03 & 0.91 & 95.63 & 2.87 & 0.07 & 95.90 & 4.26 & 0.18 \\
\hline 14 & 84.38 & 1.92 & 2.62 & 87.44 & 3.00 & 1.24 & 77.92 & 3.15 & 7.24 & 95.08 & 2.71 & 2.07 & 95.52 & 4.58 & 3.76 \\
\hline 15 & 82.47 & 1.92 & 3.01 & 84.50 & 3.15 & 4.53 & 81.56 & 2.87 & 3.88 & 94.89 & 1.92 & 3.61 & 96.49 & 4.04 & 1.04 \\
\hline
\end{tabular}

(ii) the 95-percentile modified Hausdorff distance $\left(\mathrm{H}_{95}\right)$ [93], and (iii) the absolute volume difference (AVD). These metrics are elaborated in Chapter II. Metrics were computed by comparing a ground truth segmentation to results from the proposed approach. The detailed classification results of the different brain tissues for the fifteen data sets are shown in Table 2. The average D for segmentation of the WM, GM, CSF, brain (WM+GM), and intracranial volume (ICV) (WM+GM+CSF) are $87.9 \pm 2.0 \%, 83.0 \pm 1.5 \%$, and $78.9 \pm 4.2 \%$, respectively, confirm the high accuracy of the proposed segmentation technique.

To further asses the accuracy and computational performance of the proposed brain tissue segmentation approach, it has been compared to the current state of the art segmentation approaches by participating in MICCAI Grand Challenge on MR Brain Image Segmentation (MRBrainS13) [130], which were held in the International Conference on Medical Image Computing and ComputerAssisted Intervention (MICCAI'13), Nagoya, Japan, September 2013. The challenge used twelve off-site and three on-site test data sets. The proposed approach 

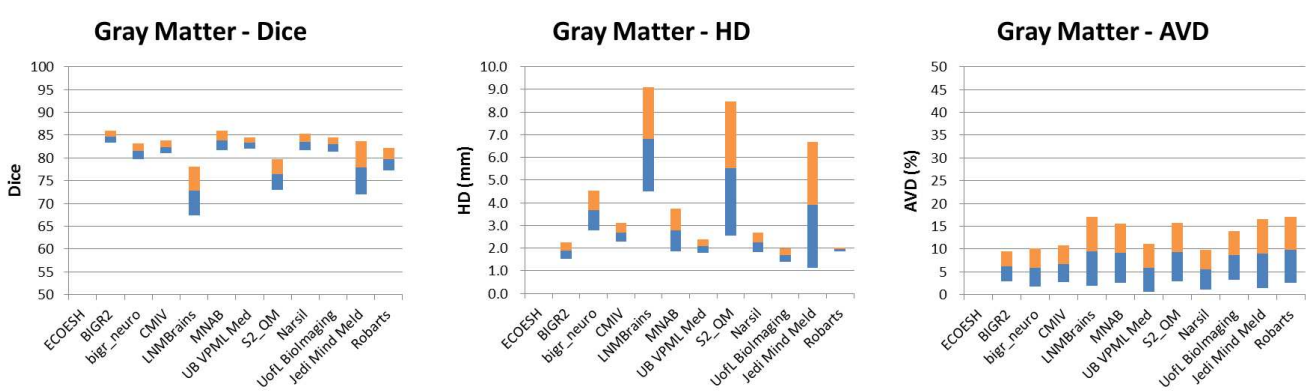

Figure 38. Evaluation results of the gray matter segmentation over all 15 scans per approach per measure. Courtesy of [130].

in this thesis has received the first prize for the best performance and has been published in the MIDAS Journal [131]. The final ranking was based on the evaluation results for both the on-site and off-set data sets. The data sets were comprehensively evaluated using the $\mathrm{D}, \mathrm{H}_{95}$ and AVD for the WM, GM, CSF tissues in the human brain. Each approach received a rank $(r), 1$ being best and 11 being worst, for each of the tissue types based on the average of the evaluation metrics. The final score (s) was determined by adding up the total scores for each approach, and the approach with the lowest score was the international winner of the MRBrainS13 challenge. As shown in Table 3, the proposed brain tissue segmentation approach has been ranked the first.

The experimental results show that the proposed accurate identification of the Joint MGRF model demonstrates promising results in segmenting the brain $(\mathrm{GM}+\mathrm{WM})$ from T1-weighted MR brain images. The present implementation in $\mathrm{C}++$ programming language on a Dell precession T7500 workstation with an Intel quad-core processor (3.33 GHz each) with $48 \mathrm{~GB}$ of memory and a $1.5 \mathrm{~TB}$ hard drive with RAID technology takes about $5.78 \pm 0.54$ seconds for processing 48 T1-MR images of size $240 \times 240$ pixels each, i.e about 0.12 seconds per image. Box-plots drawn in figures 38, 39, 40, 41, and 42 present the evaluation results of the GM, WM, CSF, brain and ICV segmentation for each of the participating algorithms over all the 15 test datasets. 
TABLE 3. Results of the 11 evaluated algorithms on the 15 test datasets. The algorithms are ranked $(r)$ based on their overall score $(s)$. This score is based on the gray matter (GM), white matter (WM) and cerebrospinal fluid (CSF) segmentation rank and the three evaluated measures: Dice coefficient (D), 95th percentile Hausdorff distance $\left(\mathrm{H}_{95}\right.$ in $\left.\mathrm{mm}\right)$, and the absolute volume difference (AVD in \%). The average runtime $t$ in seconds (s), minutes (m), or hours (h) and the scans (T1: T1-weighted scan, 3D T1: 3D T1-weighted scan, IR:T1-weighted inversion recovery (IR), F: T1-weighted FLAIR) that are used for processing are provided as well. Courtesy of [130].

\begin{tabular}{|c|c|c|c|c|c|c|c|c|c|c|c|c|c|c|c|c|c|c|}
\hline \multirow[b]{2}{*}{$r$} & \multirow{2}{*}{$\begin{array}{l}\text { Team } \\
\text { Name }\end{array}$} & \multirow[b]{2}{*}{$t$} & \multirow{2}{*}{ Scans } & \multicolumn{3}{|c|}{ GM } & \multicolumn{3}{|c|}{ WM } & \multicolumn{3}{|c|}{ CSF } & \multicolumn{3}{|c|}{ Brain } & \multicolumn{3}{|c|}{ ICV } \\
\hline & & & & $\begin{array}{c}D \\
\mu(\sigma)\end{array}$ & $\begin{array}{c}H_{95} \\
\mu(\sigma)\end{array}$ & $\begin{array}{l}\text { AVD } \\
\mu(\sigma)\end{array}$ & $\begin{array}{c}D \\
\mu(\sigma)\end{array}$ & $\begin{array}{c}H_{95} \\
\mu(\sigma)\end{array}$ & $\begin{array}{l}A V D \\
\mu(\sigma)\end{array}$ & $\begin{array}{c}D \\
\mu(\sigma)\end{array}$ & $\begin{array}{l}H_{95} \\
\mu(\sigma)\end{array}$ & $\begin{array}{l}A V D \\
\mu(\sigma)\end{array}$ & $\begin{array}{c}D \\
\mu(\sigma)\end{array}$ & $\begin{array}{c}H_{95} \\
\mu(\sigma)\end{array}$ & $\begin{array}{l}A V D \\
\mu(\sigma)\end{array}$ & $\begin{array}{c}D \\
\mu(\sigma)\end{array}$ & $\begin{array}{c}H_{95} \\
\mu(\sigma)\end{array}$ & $\begin{array}{l}\text { AVD } \\
\mu(\sigma)\end{array}$ \\
\hline 1 & $\begin{array}{c}\text { UofL } \\
\text { BioImaging }\end{array}$ & $6 s$ & T1 & $\begin{array}{l}83.0 \\
(1.5)\end{array}$ & $\begin{array}{c}1.7 \\
(0.3)\end{array}$ & $\begin{array}{c}8.6 \\
(5.4)\end{array}$ & $\begin{array}{l}87.9 \\
(2.0)\end{array}$ & $\begin{array}{c}2.2 \\
(0.6)\end{array}$ & $\begin{array}{c}8.7 \\
(6.6)\end{array}$ & $\begin{array}{l}78.9 \\
(4.2)\end{array}$ & $\begin{array}{c}2.7 \\
(0.5)\end{array}$ & $\begin{array}{l}9.7 \\
(10)\end{array}$ & $\begin{array}{l}94.9 \\
(0.6)\end{array}$ & $\begin{array}{c}2.4 \\
(0.5)\end{array}$ & $\begin{array}{c}3.9 \\
(2.0)\end{array}$ & $\begin{array}{l}96.7 \\
(0.8)\end{array}$ & $\begin{array}{c}3.4 \\
(0.6)\end{array}$ & $\begin{array}{l}1.8 \\
(2.0)\end{array}$ \\
\hline 2 & BIGR2 & $35 \mathrm{~m}$ & $\mathrm{~T} 1, \mathrm{IR}, \mathrm{F}$ & $\begin{array}{l}84.7 \\
(1.3)\end{array}$ & $\begin{array}{c}1.9 \\
(0.4)\end{array}$ & $\begin{array}{c}6.1 \\
(3.3)\end{array}$ & $\begin{array}{l}88.4 \\
(1.2)\end{array}$ & $\begin{array}{c}2.4 \\
(0.5)\end{array}$ & $\begin{array}{c}6.0 \\
(5.1)\end{array}$ & $\begin{array}{l}78.3 \\
(5.0)\end{array}$ & $\begin{array}{c}3.2 \\
(0.8)\end{array}$ & $\begin{array}{c}23 \\
(17)\end{array}$ & $\begin{array}{l}95.1 \\
(0.5)\end{array}$ & $\begin{array}{c}2.7 \\
(0.8)\end{array}$ & $\begin{array}{l}3.2 \\
(1.6)\end{array}$ & $\begin{array}{l}96.0 \\
(1.3)\end{array}$ & $\begin{array}{c}3.9 \\
(1.1)\end{array}$ & $\begin{array}{c}5.2 \\
(3.0)\end{array}$ \\
\hline 3 & CMIV & $3 \mathrm{~m}$ & $\mathrm{~T} 1, \mathrm{~F}$ & $\begin{array}{l}82.4 \\
(1.4)\end{array}$ & $\begin{array}{c}2.7 \\
(0.4)\end{array}$ & $\begin{array}{c}6.8 \\
(4.0)\end{array}$ & $\begin{array}{l}87.7 \\
(1.6)\end{array}$ & $\begin{array}{c}2.4 \\
(0.4)\end{array}$ & $\begin{array}{c}7.3 \\
(3.8)\end{array}$ & $\begin{array}{l}78.6 \\
(3.1)\end{array}$ & $\begin{array}{c}3.0 \\
(0.4)\end{array}$ & $\begin{array}{c}14 \\
(5.9)\end{array}$ & $\begin{array}{l}94.5 \\
(0.5)\end{array}$ & $\begin{array}{c}3.8 \\
(1.1)\end{array}$ & $\begin{array}{c}2.6 \\
(2.2)\end{array}$ & $\begin{array}{l}96.8 \\
(0.8)\end{array}$ & $\begin{array}{c}3.8 \\
(1.3)\end{array}$ & $\begin{array}{c}4.9 \\
(2.3)\end{array}$ \\
\hline 4 & $\begin{array}{l}\text { UB VPML } \\
\text { Med }\end{array}$ & $30 \mathrm{~m}$ & $\mathrm{~T} 1, \mathrm{IR}, \mathrm{F}$ & $\begin{array}{l}83.3 \\
(1.3)\end{array}$ & $\begin{array}{c}2.1 \\
(0.3)\end{array}$ & $\begin{array}{c}5.9 \\
(5.3)\end{array}$ & $\begin{array}{l}88.6 \\
(1.7)\end{array}$ & $\begin{array}{c}2.7 \\
(0.4)\end{array}$ & $\begin{array}{c}7.1 \\
(3.8)\end{array}$ & $\begin{array}{l}74.8 \\
(7.1)\end{array}$ & $\begin{array}{c}4.3 \\
(1.7)\end{array}$ & $\begin{array}{c}31 \\
(19)\end{array}$ & $\begin{array}{l}94.6 \\
(0.6)\end{array}$ & $\begin{array}{c}2.8 \\
(0.4)\end{array}$ & $\begin{array}{c}2.4 \\
(1.8)\end{array}$ & $\begin{array}{l}94.8 \\
(2.0)\end{array}$ & $\begin{array}{c}6.6 \\
(2.0)\end{array}$ & $\begin{array}{c}7.7 \\
(4.1)\end{array}$ \\
\hline 5 & bigr_neuro & $2 \mathrm{~h}$ & $\mathrm{~T} 1, \mathrm{~F}$ & $\begin{array}{l}81.5 \\
(1.7)\end{array}$ & $\begin{array}{c}3.7 \\
(0.9)\end{array}$ & $\begin{array}{c}5.9 \\
(4.2)\end{array}$ & $\begin{array}{l}87.3 \\
(1.4)\end{array}$ & $\begin{array}{c}3.0 \\
(0.4)\end{array}$ & $\begin{array}{c}7.3 \\
(3.8)\end{array}$ & $\begin{array}{l}78.2 \\
(4.7)\end{array}$ & $\begin{array}{c}3.2 \\
(0.6)\end{array}$ & $\begin{array}{c}16 \\
(14)\end{array}$ & $\begin{array}{l}94.0 \\
(0.8)\end{array}$ & $\begin{array}{c}4.6 \\
(1.4)\end{array}$ & $\begin{array}{c}3.6 \\
(2.4)\end{array}$ & $\begin{array}{l}96.3 \\
(1.2)\end{array}$ & $\begin{array}{c}3.9 \\
(0.9)\end{array}$ & $\begin{array}{c}3.5 \\
(2.7)\end{array}$ \\
\hline 7 & Robarts & $16 \mathrm{~m}$ & $\begin{array}{c}\text { 3D T1, } \\
\text { IR }\end{array}$ & $\begin{array}{l}79.7 \\
(2.4)\end{array}$ & $\begin{array}{c}2.0 \\
(0.1)\end{array}$ & $\begin{array}{c}9.8 \\
(7.3)\end{array}$ & $\begin{array}{l}86.2 \\
(1.3)\end{array}$ & $\begin{array}{c}3.1 \\
(0.4)\end{array}$ & $\begin{array}{c}7.1 \\
(6.2)\end{array}$ & $\begin{array}{l}80.3 \\
(4.1)\end{array}$ & $\begin{array}{c}2.7 \\
(0.5)\end{array}$ & $\begin{array}{l}20 \\
(13)\end{array}$ & $\begin{array}{l}93.1 \\
(1.6)\end{array}$ & $\begin{array}{c}2.8 \\
(0.5)\end{array}$ & $\begin{array}{c}7.9 \\
(3.6)\end{array}$ & $\begin{array}{l}97.9 \\
(0.3)\end{array}$ & $\begin{array}{c}2.6 \\
(0.4)\end{array}$ & $\begin{array}{c}0.9 \\
(0.7)\end{array}$ \\
\hline 8 & MNAB & $15 \mathrm{~m}$ & $\mathrm{~T} 1, \mathrm{IR}, \mathrm{F}$ & $\begin{array}{l}83.9 \\
(2.1)\end{array}$ & $\begin{array}{l}2.8 \\
(0.9)\end{array}$ & $\begin{array}{c}9.1 \\
(6.5)\end{array}$ & $\begin{array}{l}88.0 \\
(1.2)\end{array}$ & $\begin{array}{l}2.7 \\
(0.9)\end{array}$ & $\begin{array}{c}7.8 \\
(4.0)\end{array}$ & $\begin{array}{l}68.1 \\
(4.0)\end{array}$ & $\begin{array}{c}4.9 \\
(2.2)\end{array}$ & $\begin{array}{l}29 \\
(2 I)\end{array}$ & $\begin{array}{l}94.5 \\
(1.0)\end{array}$ & $\begin{array}{c}4.5 \\
(2.0)\end{array}$ & $\begin{array}{c}3.8 \\
(3.2)\end{array}$ & $\begin{array}{l}92.5 \\
(1 . I)\end{array}$ & $\begin{array}{c}7.1 \\
(4.2)\end{array}$ & $\begin{array}{l}9.7 \\
(4.7)\end{array}$ \\
\hline 9 & $\begin{array}{l}\text { Jedi Mind } \\
\text { Meld }\end{array}$ & $27 \mathrm{~s}$ & $\mathrm{~T} 1, \mathrm{IR}, \mathrm{F}$ & $\begin{array}{l}77.8 \\
(5.9)\end{array}$ & $\begin{array}{c}3.9 \\
(2.8)\end{array}$ & $\begin{array}{c}8.9 \\
(7.6)\end{array}$ & $\begin{array}{l}80.6 \\
(9.6)\end{array}$ & $\begin{array}{c}4.5 \\
(3.6)\end{array}$ & $\begin{array}{l}13.7 \\
(12)\end{array}$ & $\begin{array}{r}73.3 \\
(3.5)\end{array}$ & $\begin{array}{c}3.7 \\
(0.8)\end{array}$ & $\begin{array}{c}18 \\
(9.0)\end{array}$ & $\begin{array}{l}93.2 \\
(1.7)\end{array}$ & $\begin{array}{c}5.4 \\
(4.8)\end{array}$ & $\begin{array}{c}3.5 \\
(3.0)\end{array}$ & $\begin{array}{l}94.3 \\
(1.7)\end{array}$ & $\begin{array}{c}20 \\
(3.7)\end{array}$ & $\begin{array}{c}6.8 \\
(3.1)\end{array}$ \\
\hline 10 & S2_QM & $1.5 \mathrm{~h}$ & $\mathrm{~T} 1, \mathrm{IR}, \mathrm{F}$ & $\begin{array}{l}76.4 \\
(3.4)\end{array}$ & $\begin{array}{c}5.5 \\
(3.0)\end{array}$ & $\begin{array}{c}9.3 \\
(6.4)\end{array}$ & $\begin{array}{l}83.9 \\
(3.4)\end{array}$ & $\begin{array}{c}4.9 \\
(3.2)\end{array}$ & $\begin{array}{l}24.8 \\
(10)\end{array}$ & $\begin{array}{l}67.9 \\
(2.3)\end{array}$ & $\begin{array}{c}3.8 \\
(0.6)\end{array}$ & $\begin{array}{c}14 \\
(9.9)\end{array}$ & $\begin{array}{l}93.3 \\
(1.4)\end{array}$ & $\begin{array}{c}7.0 \\
(3.5)\end{array}$ & $\begin{array}{c}5.5 \\
(4.5)\end{array}$ & $\begin{array}{l}93.7 \\
(1 . I)\end{array}$ & $\begin{array}{c}6.5 \\
(1.4)\end{array}$ & $\begin{array}{l}4.0 \\
(2.2)\end{array}$ \\
\hline 11 & LNMBrains & $5 \mathrm{~m}$ & $\mathrm{~T} 1, \mathrm{IR}, \mathrm{F}$ & $\begin{array}{l}72.8 \\
(5.3)\end{array}$ & $\begin{array}{c}6.8 \\
(2.3)\end{array}$ & $\begin{array}{c}9.5 \\
(7.6)\end{array}$ & $\begin{array}{l}78.3 \\
(6.4)\end{array}$ & $\begin{array}{c}6.8 \\
(3.5)\end{array}$ & $\begin{array}{l}15.3 \\
(13)\end{array}$ & $\begin{array}{l}68.8 \\
(6.6)\end{array}$ & $\begin{array}{c}7.7 \\
(2.4)\end{array}$ & $\begin{array}{c}20 \\
(13)\end{array}$ & $\begin{array}{l}88.5 \\
(4.5)\end{array}$ & $\begin{array}{c}8.6 \\
(2.8)\end{array}$ & $\begin{array}{c}7.4 \\
(7.7)\end{array}$ & $\begin{array}{l}89.2 \\
(4.8)\end{array}$ & $\begin{array}{l}20.4 \\
(3.7)\end{array}$ & $\begin{array}{c}7.9 \\
(8.3)\end{array}$ \\
\hline
\end{tabular}



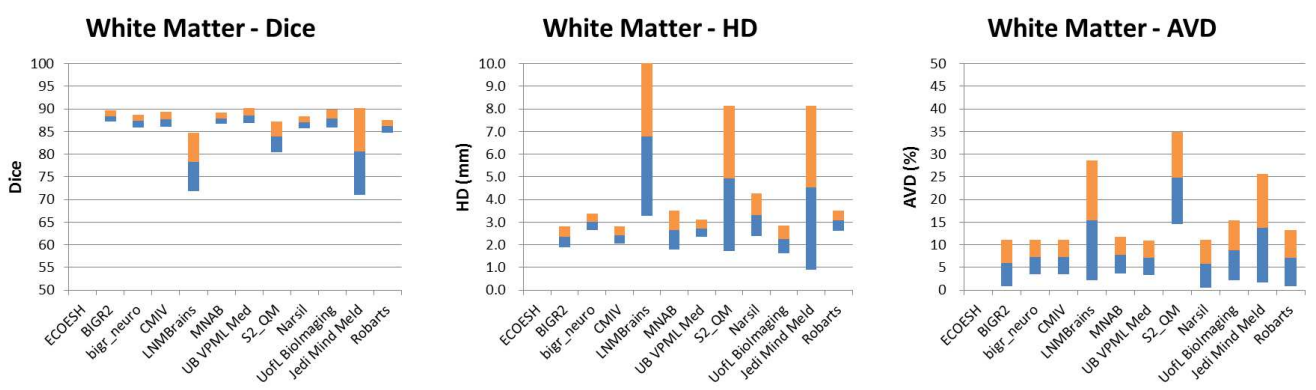

Figure 39. Evaluation results of the white matter segmentation over all 15 scans per approach per measure. Courtesy of [130].
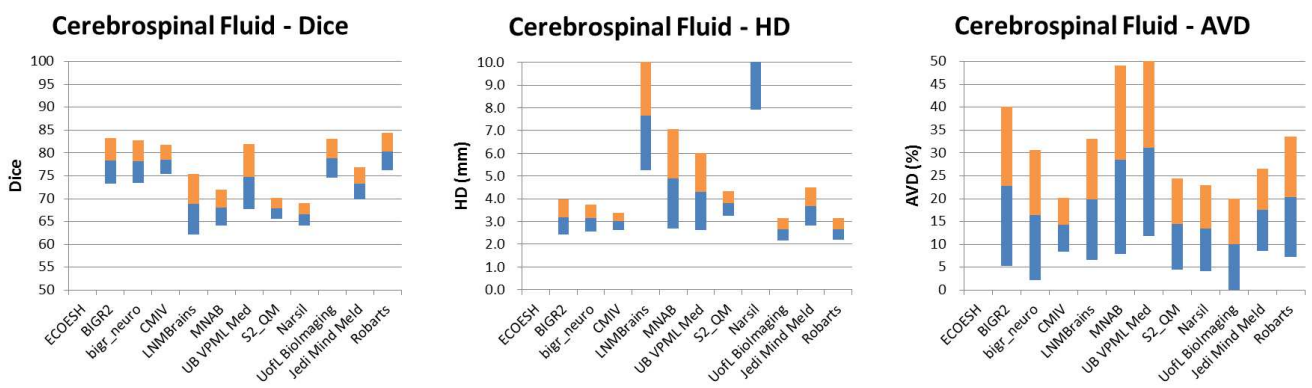

Figure 40. Evaluation results of the cerebrospinal fluid segmentation over all 15 scans per approach per measure. Courtesy of [130].
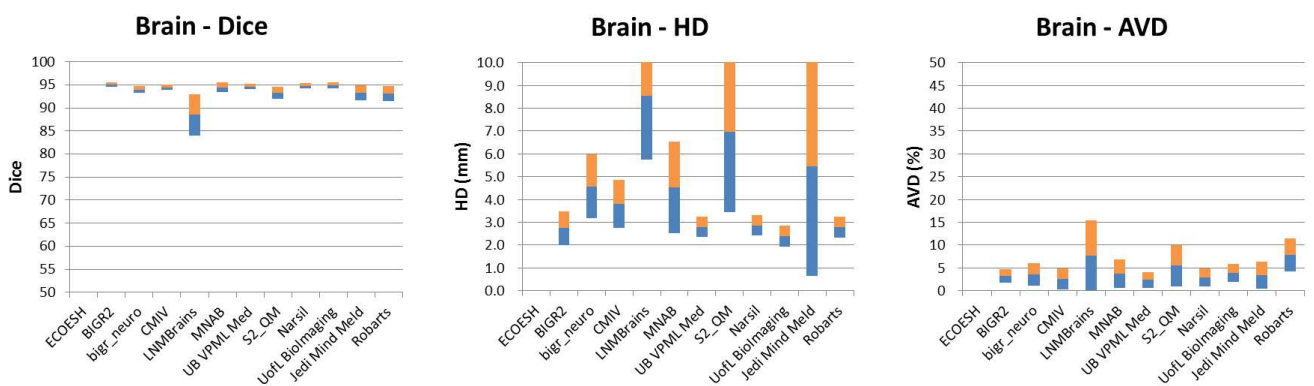

Figure 41. Evaluation results of the brain (GM and WM) segmentation over all 15 scans per approach per measure. Courtesy of [130]. 

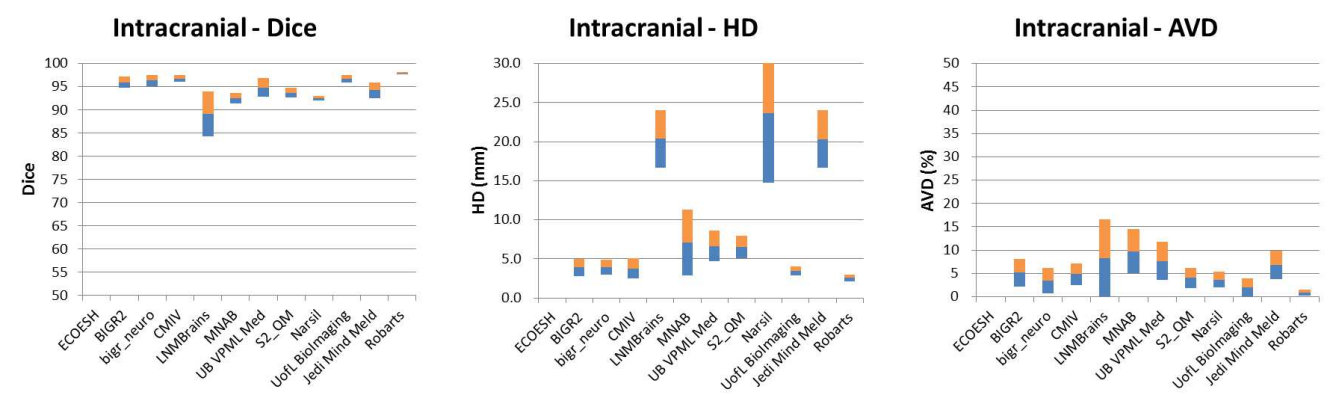

Figure 42. Evaluation results of the intracranial (GM, WM, and CSF) segmentation over all 15 scans per approach per measure. Courtesy of [130].

After validating the proposed brain tissue segmentation approach accuracy and robustness by testing it on MR adult brains and comparing it with the current state of the art segmentation approaches, the approach has been applied to T1weighted infant MR brain data sets obtained from the infant brain imaging study (IBIS) [94], and has been evaluated using dataset with manually segmented ten ground truth subjects, obtained by an MR expert. MR data was acquired at 3T images of infants scanned at approximately six months old with a voxel size of $1 \times 1 \times 1 \mathrm{~mm}^{3}$. The proposed method has segmented the infant MR brain images into three classes: brain (WM+GM), CSF, and other brain tissue. Examples of the proposed infant brain segmentation approach results are shown in figures 43,44 , and 45.

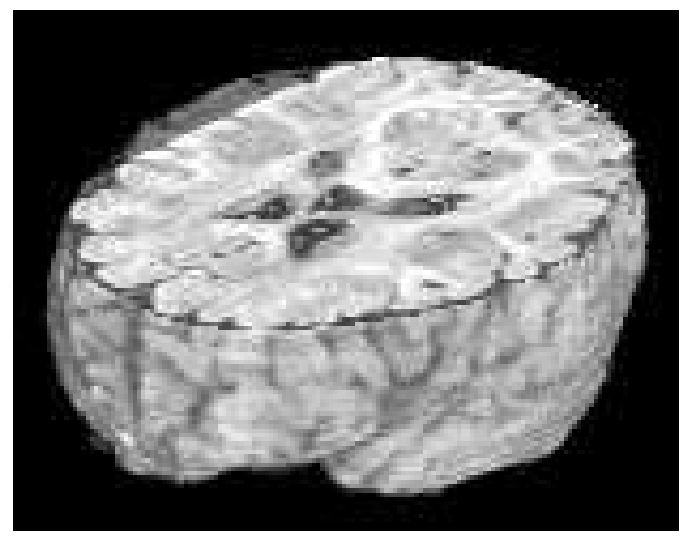

Figure 43. 3D visualization of the extracted MR infant brain. 


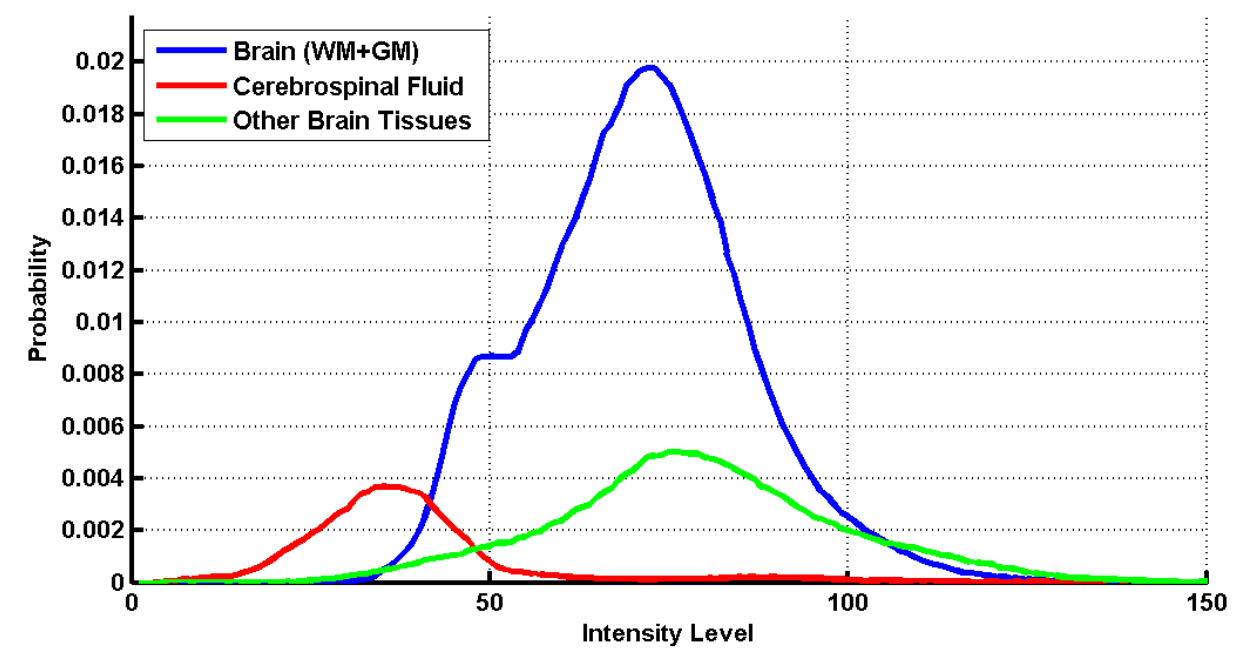

Figure 44. Normalized empirical density for each class of the infant MR brain.

The performance of the proposed segmentation framework has been evaluated using the same three performance metrics used for the adult brain segmentation: $\mathrm{D}$ [92], $\mathrm{H}_{95}$ [93], and AVD. Metrics were computed by comparing a ground truth segmentation to results from the proposed approach using 10 infant brain datasets. As demonstrated in Table 4, the average $\mathrm{D}, \mathrm{H}_{95}$, and AVD for segmentation of the brain are $89.9 \pm 3.19 \%, 6.74 \pm 2 \%$, and $9.78 \pm 8.5 \%$, respectively, which confirms the high accuracy of the proposed segmentation technique.

\section{Summary}

A new framework has been introduced in this Chapter for the automated segmentation of the brain from 3D infant MR images. The proposed approach has shown that the integration of the higher-order MGRF spatial model with the firstorder visual appearance features is a promising approach for guiding an adaptive shape model to segment T1-weighted infant brain MRIs. As mentioned in Chapter I, the ultimate goal is to integrate the proposed approach into a CAD system for diagnosing autism in infants. Another future extension of this work would be 
TABLE 4. Accuracy of the proposed segmentation approach for MR infant brain images using $\mathrm{D}(\%), \mathrm{H}_{95}(\mathrm{~mm})$, and $\mathrm{AVD}(\%)$. All metrics are represented as Mean \pm Standard Deviation.

\begin{tabular}{|c|c|c|c|}
\hline \multirow{2}{*}{ no. } & \multicolumn{3}{|c|}{ Brain } \\
\hline & D & $H_{95}$ & $A V D$ \\
\hline 1 & 91.74 & 5.7446 & 3.2859 \\
\hline 2 & 93.1 & 6 & 2.6563 \\
\hline 3 & 93.19 & 5 & 3.4969 \\
\hline 4 & 92.23 & 7.6158 & 6.3143 \\
\hline 5 & 89.41 & 7.2801 & 6.2042 \\
\hline 6 & 87.94 & 8.3666 & 4.5469 \\
\hline 7 & 90.1 & 5.099 & 10.9969 \\
\hline 8 & 82.53 & 11.4018 & 28.3425 \\
\hline 9 & 90.54 & 5.1962 & 11.3348 \\
\hline 10 & 88.26 & 5.7446 & 20.6052 \\
\hline Average & 89.904 & 6.74487 & 9.77839 \\
\hline
\end{tabular}

analyzing the classified brain tissues and testing these measurements to characterize physiological processes and disease entities or to characterize disease severity. Moreover, the proposed framework can be integrated with other methodologies to explore extracting features from the classified GM and WM regions of the brain. In particular, the accuracy of the proposed framework can help researchers to develop new techniques that can help to differentiate between autistic and control infants brains. 

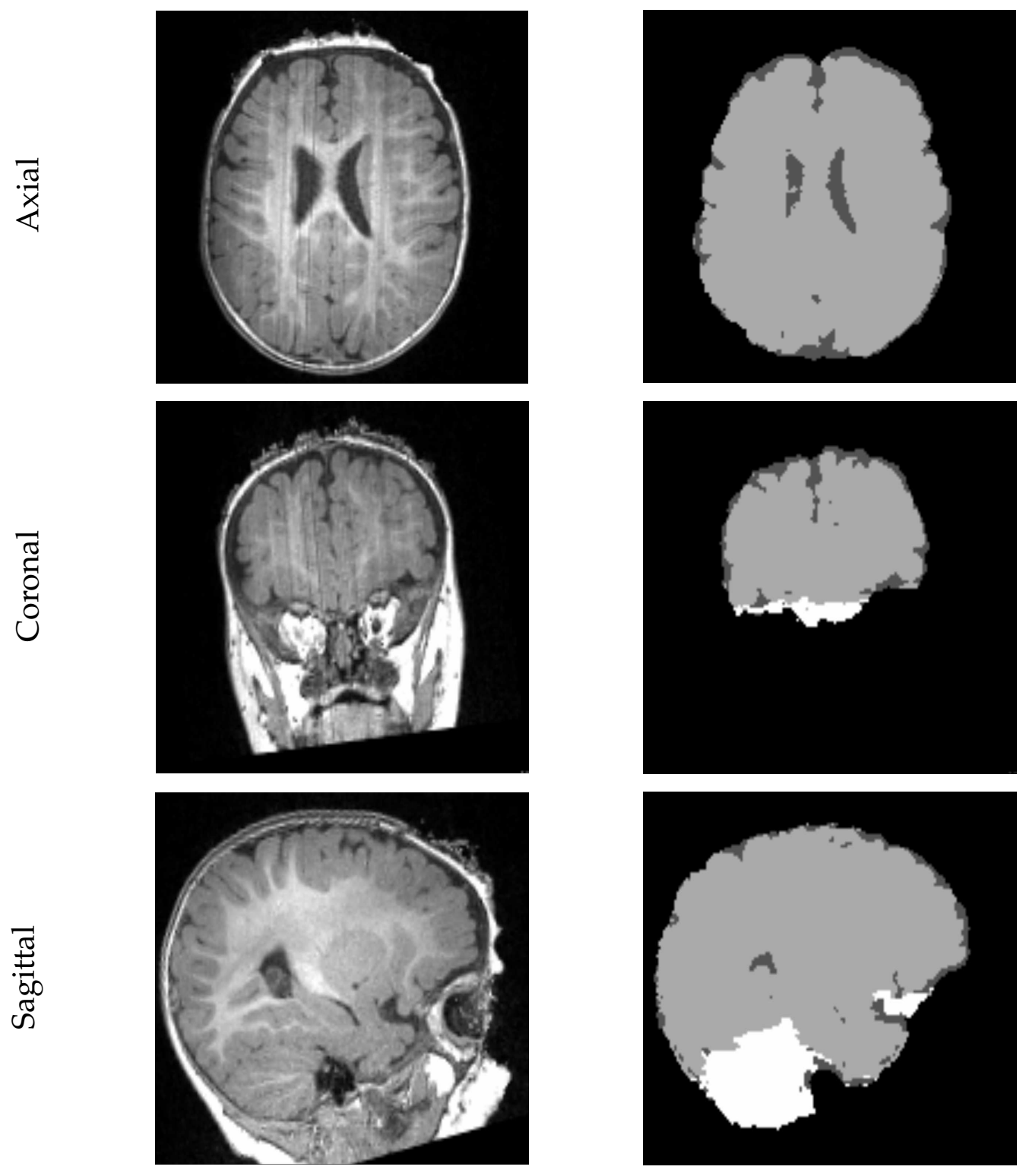

(a)

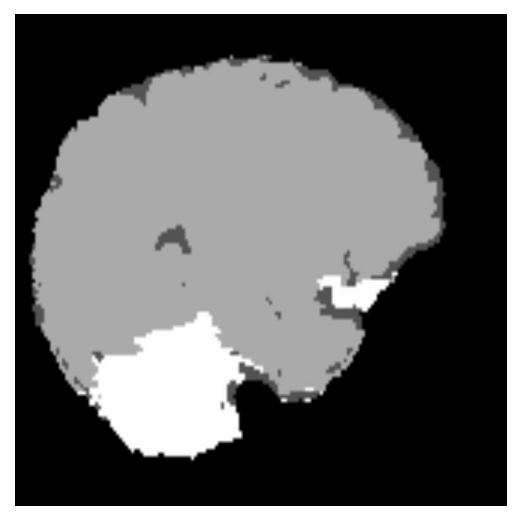

(b)

Figure 45. Segmentation is performed in 3D and the results are projected onto 2D planes for visualization. (a) The 2D profile of the original infant MRIs and (b) the final segmentation results obtained using the proposed approach. 


\section{CHAPTER IV}

\section{CONCLUSION AND FUTURE WORK}

This thesis has presented a fast and accurate classification from T1-weighted structural magnetic resonance imaging (MRI). In general, segmenting infant brain tissues from MRI contributes much to the analysis and treatment of brain injury and disorder resulting from the infant brain prematurity. Therefore, the next step will be to analyze the classified brain structures to be used in developing a computer-aided diagnosis (CAD) system for autism, which is the case study in this thesis. The proposed autism CAD system in this work consists of three main steps: (i) infant brain tissue classification from medical images, (ii) extraction of discriminatory features (e.g., shape features, WM thickness, cortical volume, etc) for the segmented brain tissues, and (iii) classification of autistic from normal infant brains based on analyzing the extracted features and shapes of different brain tissues for both normal and autistic brains. This thesis focus mainly on step (i). The proposed models and techniques developed in thesis show promising results for a variety of medical applications:

- Modeling the shape of complex medical structures such as white matter (WM), grey matter (GM), and cerebrospinal fluid (CSF) [131].

- Automatic extraction of the brain from structural MRI [132].

- Automatic classification of the brain from structural MRI into corresponding tissues (i.e., WM, GM, and CSF) [131]. 
In the following section, a summary of the most important contributions in this thesis is presented.

\section{A Contributions}

- This thesis presents a novel adaptive shape model that has the ability to capture the shape variation of complex brain structures, such as white matter $(\mathrm{WM})$, gray matter (GM), and cerebrospinal fluid (CSF), by accounting for the first- and second-order visual appearance characteristics of the medical structures. The adaptive shape model has been successfully used to guide the classification of brain tissue and has shown an ability to account for the complexity of the brain structures [131]. The preliminary results of this model confirm its benefits and encourage using it to model other medical structures.

- This thesis proposes a novel brain extraction and skull stripping [132] approach of the human brain from 3D structural MR images, which has the ability to extract both adult as well as infant brains from MRI images. The main contribution in this approach is the integration of a stochastic model (a two-level Markov-Gibbs random field (MGRF)) that serves to learn the visual appearance of the brain texture, and a geometric model (the brain iso-surfaces) that preserves the brain geometry during the extraction process. The proposed approach has been evaluated using the Dice similarity coefficient (D) [92], 95-percentile modified Hausdorff distance $\left(\mathrm{H}_{95}\right)$ [93], and the absolute volume difference (AVD) and, has been compared with widely-used brain extraction tools: infant brain extraction and analysis toolbox (iBEAT) [95], brain surface extractor (BSE) [82], brain extraction tool (BET) [70], and BET2 [96]. The experiments show that the fusion of stochas-

tic and geometric models of the brain MRI data has led to more accurate brain extraction, when compared with other widely-used brain extraction 
tools $[70,82,95,96]$.

- This thesis proposes a novel brain tissue segmentation [131] approach to classify the brain tissues into corresponding structures (i.e., WM, GM, CSF). The proposed method is based on using the developed shape prior model built using a subset of co-aligned training images that is adapted during the segmentation process based on first- and second-order visual appearance characteristics. The proposed method has been tested on both adults and infant brain MR images and the performance has been evaluated using three performance metrics: D [92], $\mathrm{H}_{95}$ [93], and absolute volume difference (AVD). The experiments of integrating a higher-order MGRF spatial model with first-order visual appearance features for guiding an adaptive shape model to segment T1-weighted infant brain MRIs have shown promising results and have documented a significant improvement in accuracy compared to the FMRIBs automated segmentation tool (FAST) [133]. Moreover, the proposed approach participated in the MICCAI Grand Challenge on MR Brain Image Segmentation (MRBrainS13) [130] on double-blind data, which were held in the International Conference on Medical Image Computing and Computer-Assisted Intervention (MICCAI'13), Nagoya, Japan, September 2013. The proposed approach in this thesis has achieved the first prize and has been published in the MIDAS Journal [131].

\section{B Future Work}

The work presented in this thesis can be further enhanced and extended as follows:

- The proposed brain classification framework has been used to segment WM, GM, and CSF brain structures. Future work include investigating the ability of the proposed method to segment other brain structures, such as the corpus 
callosum (CC), the hippocampus, and the cerebellum.

- This thesis presents an accurate method for brain tissue classification into the corresponding brain structures (i.e., GM, WM, CSF). The promising accuracy of the proposed framework can help researchers to develop new techniques that can help to differentiate between autistic and control infants brains. Future work explores extracting diagnostic features in the segmented GM and WM regions of the brain and integrate these methods in the developed CAD system for autism. In order to enhance the diagnostic accuracy, future work will investigate integrating other diagnostic features that will be extracted from other brain structures, such as the corpus callosum (CC), the hippocampus, and the cerebellum.

- The ultimate goal of the work proposed in this thesis is to develop a CAD system for early autism diagnosis. A future extension of this work would be analyzing the extracted brain and testing these measurements not only to diagnose autism [134-141], but also to characterize physiological processes and other disease entities or to characterize the severity of other diseases such as dyslexia $[142,143]$, brain tumors, strokes, seizure disorders, depression, and Alzheimer's disease [33].

- Another future work will be to apply the developed models in other clinical applications such as: acute renal rejection [144-153], lung cancer detection [154-187], and cancerous cells detection in the prostate [188-194]. 


\section{REFERENCES}

[1] P. Mansfield. Imaging by nuclear magnetic resonance. Journal Physics E: Science Instrumentaion, 21:18-30, 1984.

[2] P. Mansfield. Snapshot magnetic resonance imaging (nobel lecture). Angewandte Chemie International Edition, 43(41):5456-5464, 2004.

[3] E. M. Haacke. Magnetic Resonance Imaging: Physical Principles and Sequence Design. J. Wiley \& Sons, New York, USA, 1999.

[4] Medical skepticism, vol.5. http://glasshospital.com/2011/10/30/ medical-skepticism-vol-5/.

[5] F. W. Wehrli, J. MacFall, T. H. Newton, and D. G. Potts. Advanced Imaging Techniques. Clavadel Press, San Francisco, CA, USA, 1983.

[6] W. G. Bradley. Effect of magnetic relaxation times on magnetic resonance image interpretation. Noninvasive Medical Imaging, 1:193-204, 1984.

[7] A. G. Webb. Introduction to Biomedical Imaging. Wiley-IEEE Press, NJ, USA, 2003.

[8] H. Michaely, K. Herrmann, K. Nael, N. Oesingmann, M. Reiser, and S. Schoenberg. Functional renal imaging: Nonvascular renal disease. $A b-$ dominal Imaging, 32(1):1-16, 2007.

[9] D. J. Collins and A. R. Padhani. Dynamic magnetic resonance imaging of tumor perfusion. IEEE Engineering in Medicine and Biology Magazine, 23(5):65$83,2004$. 
[10] F. Khalifa, G. M. Beache, G. Gimel'farb, and A. El-Baz. A novel cad system for analyzing cardiac first-pass MRI images. In Proceedings International Conference on Pattern Recognition, ICPR-2012, pages 77-80, Tsukuba Science City, Japan, November 11-15, 2012.

[11] A. El-Baz, A. Farag, R. Fahmi, S. Yuksel, M. A. El-Ghar, and T. Eldiasty. Image analysis of renal dce mri for the detection of acute renal rejection. In Proceedings International Conference on Pattern Recognition, ICPR-2006, pages 822-825, Hong Kong, August 20-24, 2006.

[12] A. Farag, A. El-Baz, S. Yuksel, M. A. El-Ghar, and T. Eldiasty. A framework for the detection of acute rejection with dynamic contrast enhanced magnetic resonance imaging. In Proceedings IEEE International Symposium on Biomedical Imaging: From Nano to Macro, ISBI-2006, pages 418-421, Arlington, Virginia, USA, April 69, 2006.

[13] A. El-Baz, A. A. Farag, S. E. Yuksel, M. E. A. El-Ghar, T. A. Eldiasty, and M. A. Ghoneim. Application of deformable models for the detection of acute renal rejection. In A. A. Farag and J. S. Suri, editors, Deformable Models, volume 1, chapter 10, pages 293-333. Springer, 2007.

[14] A. El-Baz, G. Gimel'farb, and M. A. El-Ghar. New motion correction models for automatic identification of renal transplant rejection. In Proceedings International Conference Medical Image Computing and Computer-Assisted Intervention, MICCAI-2007, pages 235-243, Brisbane, Australia, October 29 November 2, 2007.

[15] A. El-Baz, G. Gimel'farb, and M. A. El-Ghar. A novel image analysis approach for accurate identification of acute renal rejection. In Proceedings IEEE International Conference on Image Processing, ICIP-2008, pages 1812-1815, San Diego, California, USA, October 12-15, 2008. 
[16] A. El-Baz, G. Gimel'farb, and M. A. El-Ghar. Image analysis approach for identification of renal transplant rejection. In Proceedings International Conference on Pattern Recognition, ICPR-2008, pages 1-4, Tampa, Florida, USA, December 8-11, 2008.

[17] F. Khalifa, A. El-Baz, G. Gimel'farb, and M. A. El-Ghar. Non-invasive imagebased approach for early detection of acute renal rejection. In Proceedings International Conference Medical Image Computing and Computer-Assisted Intervention, MICCAI-2010, pages 10-18, Beijing, China, September 20-24, 2010.

[18] A. Firjani, F. Khalifa, A. Elnakib, G. Gimel'farb, A. Elmaghraby, and A. ElBaz. A novel image-based approach for early detection of prostate cancer. In Proceedings IEEE International Conference on Image Processing, ICIP-2012, pages 2849-2852, Lake Buena Vista, Florida, USA, 30 September- 3 October, 2012.

[19] A. Firjani, F. Khalifa, A. Elnakib, G. Gimel'farb, M. A. El-Ghar, A. Elmaghraby, and A. El-Baz. Non-invasive image-based approach for early detection of prostate cancer. In Proceedings Fourth International Conference on Developments in eSystems Engineering, DeSE-11, pages 172-177, Dubai, UAE, December 6-8, 2011.

[20] B. Sitharaman, K. R. Kissell, K. B. Hartman, L. A. Tran, A. Baikalov, I. Rusakova, Y. Sun, H. A. Khant, S. J. Ludtke, W. Chiu, S. Laus, E. Toth, L. Helm, A. E. Merbachd, and L. J. Wilson. Superparamagnetic gadonanotubes are high-performance MRI contrast agents. Chemical Communications, 1(31):3915-3917, 2006.

[21] R. Rajeshkannan, S. Moorthy, K. Sreekumar, R. Rupa, N. Prabhu, et al. Clinical applications of diffusion weighted MR imaging: A review. Indian Journal of Radiology and Imaging, 16(4):705, 2006. 
[22] P. J. Basser, J. Mattiello, and D. LeBihan. Mr diffusion tensor spectroscopy and imaging. Biophysical journal, 66(1):259-267, 1994.

[23] T. Liu, H. Li, K. Wong, A. Tarokh, L. Guo, and S. T. Wong. Brain tissue segmentation based on dti data. NeuroImage, 38(1):114-123, 2007.

[24] D. Le Bihan, S.-i. Urayama, T. Aso, T. Hanakawa, and H. Fukuyama. Direct and fast detection of neuronal activation in the human brain with diffusion mri. Proceedings of the National Academy of Sciences, 103(21):8263-8268, 2006.

[25] C. Sato, S. Naganawa, T. Nakamura, H. Kumada, S. Miura, O. Takizawa, and T. Ishigaki. Differentiation of noncancerous tissue and cancer lesions by apparent diffusion coefficient values in transition and peripheral zones of the prostate. Magn. Reson. Imaging, 21(10):258262, 2005.

[26] R. Bammer. Basic principles of diffusion-weighted imaging. European Journal Radiology, 45(43):169-184, 2003.

[27] P. C. Sundgren, Q. Dong, D. G'mez-Hassan, S. K. Mukherji, P. Maly, and R. Welsh. Diffusion tensor imaging of the brain: Review of clinical applications. Neuroradiology, 46(5):339-250, 2004.

[28] D. Le Bihan, J. F. Mangin, C. Poupon, C. A. Clark, S. Pappata, N. Molko, and H. Chabriat. Diffusion tensor imaging: concepts and applications. Journal of Magnetic Resonance Imaging, 13(4):354-546, 2001.

[29] T. Schultz. Estimating the crossing of nerve fibers in the human brain. In Proceedings SPIE Electronic Imaging E Signal Processing, SPIE-2009, pages 13, 2009.

[30] V. J. Wedeen, R. P. Wang, J. D. Schmahmann, B. T., W. Y. Tseng, G. Dai, D. N. Pandya, P. Hagmann, D. H., and de Crespigny A. J. Diffusion spectrum mag- 
netic resonance imaging (DSI) tractography of crossing fibers. Neuroimage, 41(4):1267-1277, 2008.

[31] P. Hagmann, L. Jonasson, P. Maeder, J.-P. Thiran, V. J. Wedeen, and R. Meuli. Understanding diffusion MR imaging techniques: From scalar diffusionweighted imaging to diffusion tensor imaging and beyond. Radiographics, 26(1):205-1203, 2006.

[32] S. A. Huettel, A. W. Song, and G. McCarthy. Functional magnetic resonance imaging, volume 1. Sinauer Associates Sunderland, MA, 2004.

[33] Radiologyinfo.org. The radiology information resource for patients. http: //www.radiologyinfo.org/en/info.cfm?pg=fmribrain.

[34] S. Ogawa, T. M. Lee, A. S. Nayak, and P. Glynn. Oxygenation-sensitive contrast in magnetic resonance image of rodent brain at high magnetic fields. Magn. Reson. Med., 14(1):68-78, 1990.

[35] A. Carpentier, K. Pugh, M. Westerveld, C. Studholme, O. Skrinjar, J. L. Thompson, D. D. Spencer, and R. T. Constable. Functional mri of language processing: dependence on input modality and temporal lobe epilepsy. Epilepsia, 42(10):1241-1254, 2001.

[36] C. S. Carter, A. W. Macdonald III, L. L. Ross, and V. A. Stenger. Anterior cingulate cortex activity in impaired self-monitoring of performance in patients with schizophrenia: An event-related fMRI study. American Journal of Psychiatry, 158(10):1423-1428, 2001.

[37] W. R. Staines, M. W. E., S. J. Graham, and S. E. Black. Bilateral movement enhances ipsilesional cortical activity in acute stroke: A pilot functional MRI. Neurology, 56(3):401-404, 2001. 
[38] S. Narayana and J. Xiong. Reading treatment helps children with dyslexia and changes activity in language areas of the brain. Neurology, 61(2):E5-E6, 2003.

[39] J. Jackson, D. J. Allison, and J. Meaney. Angiography: principles, techniques, and complications. In R. C. Grainger, D. Allison, A. Adam, and A. K. Dixon, editors, Diagnostic Radiology: A Textbook of Medical Imaging, chapter 6. Churchill Livingstone, New York, NY, 2008.

[40] X. Yang and K. Murase. Tagged cardiac MR image segmentation by contrast enhancement and texture analysis. International Conference on Electronic Measurement Instruments,(ICEMI '09), pages 4-210, 2009.

[41] X. Liu and J. L. Prince. Shortest path refinement for motion estimation from tagged MR Images. IEEE Transactions on Medical Imaging, 29(8):1560-1572, 2010.

[42] L. Axel and L. Dougherty. Heart wall motion: Improved method of spatial modulation of magnetization for MR imaging. Radiology, 172(8):349-350, 1989.

[43] N. Shah, A. Sattar, M. Benanti, S. Hollander, and L. Cheuck. Magnetic resonance spectroscopy as an imaging tool for cancer: a review of the literature. JAOA: Journal of the American Osteopathic Association, 106(1):23-27, 2006.

[44] A. Horská, P. B. Barker, and D. Phil. Imaging of Brain Tumors: MR Spectroscopy and Metabolic Imaging. Neuroimaging Clinics of North America, 20(3):293-310, 2010.

[45] P. Swindle, S. McCredie, P. Russell, U. Himmelreich, M. Khadra, C. Lean, and M. C. Pathologic characterization of human prostate tissue with proton MR spectroscopy. Radiology, 228(1):144-151, 2003. 
[46] L. L. Cheng, M. A. Burns, J. L. Taylor, W. He, E. F. Halpern, W. S. McDougal, and W. C. L. Metabolic characterization of human prostate cancer with tissue magnetic resonance spectroscopy. Cancer Research, 65(8):3030-3034, 2005.

[47] M. A. Jacobs, P. B. Barker, P. Argani, R. Ouwerkerk, Z. M. Bhujwalla, and B. D. A. Combined dynamic contrast enhanced breast MR and proton spectroscopic imaging: A feasibility study. Journal of Magnetic Resonance Imaging, 21(1):23-28, 2005.

[48] M. A. Jacobs, P. B. Barker, P. A. Bottomley, Z. Bhujwalla, and B. D. A. Proton magnetic resonance spectroscopy imaging of human breast cancer: a preliminary study. Journal of Magnetic Resonance Imaging, 19(1):68-75, 2004.

[49] M. M. Mahon, I. J. Cox, R. Dina, W. P. Soutter, G. A. McIndoe, A. D. Williams, and N. M. deSouza. ${ }^{1} \mathrm{H}$ magnetic resonance spectroscopy of preinvasive and invasive cervical cancer: in vivo-ex vivo profiles and effect of tumor load. Journal of Magnetic Resonance Imaging, 19(1):256-364, 2004.

[50] M. M. Mahon, A. D. Williams, W. P. Soutter, I. J. Cox, G. A. McIndoe, G. A. Coutts, R. Dina, and N. M. deSouza. ${ }^{1} \mathrm{H}$ magnetic resonance spectroscopy of invasive cervical cancer: an in vivo study with ex vivo corroboration. NMR in Biomedicine, 17(1):1-9, 2004.

[51] S. G. Cho, D. H. Lee, K. Y. Lee, H. Ji, K. H. Lee, P. R. Ros, and C. H. Suh. Differentiation of chronic focal pancreatitis from pancreatic carcinoma by in vivo proton magnetic resonance spectroscopy. Journal Computer Assisted Tomography, 29(2):163-169, 2005.

[52] S. T. Doran, G. L. Falk, R. L. Somorjai, C. L. Lean, U. Himmelreich, J. Philips, P. Russell, B. Dolenko, A. E. Nikulin, and C. E. Mountford. Pathology of barretts esophagus by proton magnetic resonance spectroscopy and a statistical classification strategy. The American Journal of Surgery, 185(3):232-238, 2003. 
[53] L. T. Morais, V. de Araújo Zanardi, and A. V. Faria. Magnetic resonance spectroscopy in the diagnosis and etiological definition of brain bacterial abscesses. Arq Neuropsiquiatr, 65(4B):1144-1148, 2007.

[54] E. L. Barbier, L. Lamalle, and M. Décorps. Methodology of brain perfusion imaging. J. Magn. Reson. Imaging, 13(4):496-520, 2001.

[55] J. Lu, K. Li, M. Zhang, and L. Jiao. Dynamic susceptibility contrast perfusion magnetic resonance imaging in patients with symptomatic unilateral middle cerebral artery stenosis or occlusion. Acta Radiology, 48(3):335-340, 2007.

[56] D. Lin, J. Kleinman, R. Wityk, R. Gottesman, A. E. Hillis, A. Lee, and P. B. Barker. Crossed cerebellar diaschisis in acute stroke detected by dynamic susceptibility contrast MR perfusion imaging. American Journal of Neuroradiology, 30(4):710-715, 2009.

[57] E. S. Paulson and K. M. Schmainda. Comparison of dynamic susceptibilityweighted contrast-enhanced MR Methods: Recommendations for measuring relative cerebral blood volume in brain tumors. Radiology, 249(2):601$613,2008$.

[58] L. Mechtler. Neuroimaging in neuro-oncology. Neurologic Clinics, 27(1):171201, 2009.

[59] A. Raznahan, R. Toro, E. Daly, D. Robertson, C. Murphy, Q. Deeley, P. F. Bolton, T. Paus, and D. G. Murphy. Cortical anatomy in autism spectrum disorder: an in vivo MRI study on the effect of age. Cerebral cortex, 20(6):13321340, 2010.

[60] A. Y. Hardan, R. A. Libove, M. S. Keshavan, N. M. Melhem, and N. J. Minshew. A preliminary longitudinal magnetic resonance imaging study of brain volume and cortical thickness in autism. Biological psychiatry, 66(4):320-326, 2009. 
[61] M. Nitzken, M. F. Casanova, F. Khalifa, G. Sokhadze, and A. El-Baz. ShapeBased Detection of Cortex Variability for More Accurate Discrimination Between Autistic and Normal Brains. Springer, 2011.

[62] D. W. Shattuck, G. Prasad, M. Mirza, K. L. Narr, and A. W. Toga. Online resource for validation of brain segmentation methods. NeuroImage, 45(2):431439, 2009.

[63] C. Fennema-Notestine et al. Quantitative evaluation of automated skullstripping methods applied to contemporary and legacy images: Effects of diagnosis, bias correction, and slice location. Human Brain Mapping, 27(2):99$113,2006$.

[64] A. J. van der Kouwe, T. Benner, D. H. Salat, and B. Fischl. Brain morphometry with multiecho mprage. Neuroimage, 40(2):559-569, 2008.

[65] F. Shi, Y. Fan, S. Tang, J. H. Gilmore, W. Lin, and D. Shen. Neonatal brain image segmentation in longitudinal MRI studies. Neuroimage, 49(1):391-400, 2010.

[66] A. U. Mewes, P. S. Hüppi, et al. Regional brain development in serial magnetic resonance imaging of low-risk preterm infants. Pediatrics, 118(1):23-33, 2006.

[67] M. A. Balafar, A. R. Ramli, M. I. Saripan, and S. Mashohor. Review of brain MRI image segmentation methods. Artificial Intelligence Review, 33(3):261$274,2010$.

[68] N. I. Weisenfeld and S. K. Warfield. Automatic segmentation of newborn brain MRI. Neuroimage, 47(2):564-572, 2009.

[69] H. Xue, L. Srinivasan, S. Jiang, M. Rutherford, A. D. Edwards, D. Rueckert, 
and J. V. Hajnal. Automatic segmentation and reconstruction of the cortex from neonatal MRI. Neuroimage, 38(3):461-477, 2007.

[70] S. M. Smith. Fast robust automated brain extraction. Human Brain Mapping, 17(3):143-155, 2002.

[71] J.-X. Liu, Y.-S. Chen, et al. Accurate and robust extraction of brain regions using a deformable model based on radial basis functions. Journal of Neuroscience Methods, 183(2):255-266, 2009.

[72] H. Wendland. Piecewise polynomial, positive definite and compactly supported radial functions of minimal degree. Advances in computational Mathematics, 4(1):389-396, 1995.

[73] A. H. Zhuang, D. J. Valentino, and A. W. Toga. Skull-stripping magnetic resonance brain images using a model-based level set. NeuroImage, 32(1):79$92,2006$.

[74] C. Baillard, P. Hellier, and C. Barillot. Segmentation of brain 3D MR images using level sets and dense registration. Medical Image Analysis, 5(3):185-194, 2001.

[75] S. A. Sadananthan, W. Zheng, M. W. Chee, and V. Zagorodnov. Skull stripping using graph cuts. NeuroImage, 49(1):225-239, 2010.

[76] H. Zhang, J. Liu, Z. Zhu, and H. Li. An automated and simple method for brain MR image extraction. Biomedical Engineering Online, 10(1):81, 2011.

[77] K. Somasundaram and T. Kalaiselvi. Automatic brain extraction methods for T1 magnetic resonance images using region labeling and morphological operations. Computers in Biology and Medicine, 41(8):716-725, 2011. 
[78] K. K. Leung, J. Barnes, M. Modat, G. R. Ridgway, J. W. Bartlett, N. C. Fox, and S. Ourselin. Brain MAPS: an automated, accurate and robust brain extraction technique using a template library. Neuroimage, 55(3):1091-1108, 2011.

[79] J. E. Iglesias, C.-Y. Liu, et al. Robust brain extraction across datasets and comparison with publicly available methods. IEEE Transactions on Medical Imaging, 30(9):1617-1634, 2011.

[80] F. Segonne, A. Dale, et al. A hybrid approach to the skull stripping problem in MRI. Neuroimage, 22(3):1060-1075, 2004.

[81] D. E. Rex, D. W. Shattuck, et al. A meta-algorithm for brain extraction in MRI. NeuroImage, 23(2):625-637, 2004.

[82] D. W. Shattuck and R. M. Leahy. Brainsuite: an automated cortical surface identification tool. Medical Image Analysis, 6(2):129-142, 2002.

[83] B. D. Ward. Intracranial segmentation. Biophysics Research Institute, Medical College of Wisconsin, Milwaukee, WI, 1999.

[84] A. M. Dale, B. Fischl, and M. I. Sereno. Cortical surface-based analysis: I. Segmentation and surface reconstruction. Neuroimage, 9(2):179-194, 1999.

[85] C. Bouman and K. Sauer. A generalized gaussian image model for edgepreserving MAP estimation. IEEE Transactions on Image Processing, 2(3):296310, 1993.

[86] N. J. Tustison, B. B. Avants, et al. N4ITK: improved N3 bias correction. IEEE Transactions on Medical Imaging, 29(6):1310-1320, 2010.

[87] J. Besag. On the statistical analysis of dirty pictures. Journal of the Royal Statistical Society. Series B (Methodological), pages 259-302, 1986. 
[88] D. Adalsteinsson. A fast level set method for propagating interfaces. PhD thesis, Citeseer, 1994.

[89] A. El-Baz et al. Precise segmentation of 3-D magnetic resonance angiography. IEEE Transactions on Biomedical Engineering, 59(7):2019-2029, 2012.

[90] A. Farag, A. El-Baz, and G. Gimel'farb. Precise segmentation of multimodal images. IEEE Transactions on Image Processing, 15(4):952-968, 2006.

[91] A. El-Baz. Novel stochastic models for medical image analysis. PhD thesis, University of Louisville, Louisville, KY, USA, 2006.

[92] D. Lee R. Measures of the amount of ecologic association between species. Ecology, 26:297-302, 1945.

[93] G. Gerig, M. Jomier, and M. Chakos. Valmet: A new validation tool for assessing and improving 3D object segmentation. In Medical Image Computing and Computer Assisted Intervention, pages 516-523, 2001.

[94] IBIS. Infant brain imaging study. http://www. ibisnetwork. org/.

[95] F. Shi, L. Wang, Y. Dai, J. H. Gilmore, W. Lin, and D. Shen. LABEL: Pediatric brain extraction using learning-based meta-algorithm. NeuroImage, 62(3):1975-1986, 2012.

[96] M. Jenkinson, M. Pechaud, and S. Smith. BET2: MR-based estimation of brain, skull and scalp surfaces. In Eleventh Annual Meeting of The Organization for Human Brain Mapping, volume 17, 2005.

[97] A. Barkovich. Magnetic resonance techniques in the assessment of myelin and myelination. Journal of inherited metabolic disease, 28(3):311-343, 2005.

[98] A. M. Mathur, J. J. Neil, R. C. McKinstry, and T. E. Inder. Transport, monitoring, and successful brain MR imaging in unsedated neonates. Pediatric radiology, 38(3):260-264, 2008. 
[99] H. Ng, S. Ong, K. Foong, P. Goh, and W. Nowinski. Medical image segmentation using K-means clustering and improved watershed algorithm. In Image Analysis and Interpretation, 2006 IEEE Southwest Symposium on, pages 61-65. IEEE, 2006.

[100] T. Song, M. M. Jamshidi, R. R. Lee, and M. Huang. A modified probabilistic neural network for partial volume segmentation in brain MR image. Neural Networks, IEEE Transactions on, 18(5):1424-1432, 2007.

[101] P. Anbeek, K. L. Vincken, F. Groenendaal, A. Koeman, M. J. Van Osch, and J. Van der Grond. Probabilistic brain tissue segmentation in neonatal magnetic resonance imaging. Pediatric research, 63(2):158-163, 2008.

[102] A. Mayer and H. Greenspan. An adaptive mean-shift framework for MRI brain segmentation. Medical Imaging, IEEE Transactions on, 28(8):1238-1250, 2009.

[103] R. Fang, Y.-h. J. Chen, R. Zabih, and T. Chen. Tree-metrics graph cuts for brain MRI segmentation with tree cutting. In Image Processing Workshop (WNYIPW), 2010 Western New York, pages 10-13. IEEE, 2010.

[104] A. Ortiz, J. Górriz, J. Ramirez, and D. Salas-Gonzalez. MR brain image segmentation by growing hierarchical SOM and probability clustering. Electronics Letters, 47(10):585-586, 2011.

[105] L. Wang, F. Shi, G. Li, W. Lin, J. H. Gilmore, and D. Shen. Integration of Sparse Multi-modality Representation and Geometrical Constraint for Isointense Infant Brain Segmentation. In Medical Image Computing and ComputerAssisted Intervention-MICCAI 2013, pages 703-710. Springer, 2013.

[106] J. Ashburner and K. J. Friston. Unified segmentation. Neuroimage, 26(3):839$851,2005$. 
[107] K. M. Pohl, J. Fisher, W. E. L. Grimson, R. Kikinis, and W. M. Wells. A bayesian model for joint segmentation and registration. Neurolmage, 31(1):228-239, 2006.

[108] X. Han and B. Fischl. Atlas renormalization for improved brain MR image segmentation across scanner platforms. Medical Imaging, IEEE Transactions on, 26(4):479-486, 2007.

[109] X. Artaechevarria, A. Munoz-Barrutia, and C. Ortiz-de Solorzano. Combination strategies in multi-atlas image segmentation: Application to brain MR data. Medical Imaging, IEEE Transactions on, 28(8):1266-1277, 2009.

[110] M. R. Sabuncu, B. T. Yeo, K. Van Leemput, B. Fischl, and P. Golland. A generative model for image segmentation based on label fusion. Medical Imaging, IEEE Transactions on, 29(10):1714-1729, 2010.

[111] J.-P. Morin, C. Desrosiers, and L. Duong. Atlas-based segmentation of brain magnetic resonance imaging using random walks. In Computer Vision and Pattern Recognition Workshops (CVPRW), 2012 IEEE Computer Society Conference on, pages 44-49. IEEE, 2012.

[112] J.-M. Morel and G. Yu. ASIFT: A new framework for fully affine invariant image comparison. SIAM Journal on Imaging Sciences, 2(2):438-469, 2009.

[113] H. Bay, T. Tuytelaars, and L. Van Gool. Surf: Speeded up robust features. In Computer Vision-ECCV 2006, pages 404-417. Springer, 2006.

[114] J. M. Lötjönen, R. Wolz, J. R. Koikkalainen, L. Thurfjell, G. Waldemar, H. Soininen, and D. Rueckert. Fast and robust multi-atlas segmentation of brain magnetic resonance images. NeuroImage, 49(3):2352-2365, 2010.

[115] F. van der Lijn, M. de Bruijne, S. Klein, T. den Heijer, Y. Y. Hoogendam, A. van der Lugt, M. M. Breteler, and W. J. Niessen. Automated brain struc- 
ture segmentation based on atlas registration and appearance models. Medical Imaging, IEEE Transactions on, 31(2):276-286, 2012.

[116] Y. Boykov, O. Veksler, and R. Zabih. Fast approximate energy minimization via graph cuts. Pattern Analysis and Machine Intelligence, IEEE Transactions on, 23(11):1222-1239, 2001.

[117] C. Ledig, R. Wolz, P. Aljabar, J. Lotjonen, R. A. Heckemann, A. Hammers, and D. Rueckert. Multi-class brain segmentation using atlas propagation and EM-based refinement. In Biomedical Imaging (ISBI), 2012 9th IEEE International Symposium on, pages 896-899. IEEE, 2012.

[118] E. D. Angelini, T. Song, B. D. Mensh, and A. F. Laine. Segmentation and quantitative evaluation of brain MRI data with a multi-phase threedimensional implicit deformable model. Medical imaging 2004: Image processing: 16-19 February 2004, San Diego, California, USA; Proceedings of SPIE, vol. 5370, 2004.

[119] O. Colliot, O. Camara, and I. Bloch. Integration of fuzzy spatial relations in deformable modelsApplication to brain MRI segmentation. Pattern recognition, 39(8):1401-1414, 2006.

[120] S. Miri, N. Passat, and J.-P. Armspach. Topology-preserving discrete deformable model: Application to multi-segmentation of brain MRI. In Image and Signal Processing, pages 67-75. Springer, 2008.

[121] A. Albert Huang, R. Abugharbieh, and R. Tam. A Hybrid GeometricStatistical Deformable Model for Automated 3-D Segmentation in Brain MRI. Biomedical Engineering, IEEE Transactions on, 56(7):1838-1848, 2009.

[122] M. Del Fresno, M. Vénere, and A. Clausse. A combined region growing and deformable model method for extraction of closed surfaces in 3D CT and MRI scans. Computerized Medical Imaging and Graphics, 33(5):369-376, 2009. 
[123] L. Wang, Y. Chen, X. Pan, X. Hong, and D. Xia. Level set segmentation of brain magnetic resonance images based on local Gaussian distribution fitting energy. Journal of neuroscience methods, 188(2):316-325, 2010.

[124] S. Bourouis and K. Hamrouni. 3D segmentation of MRI brain using level set and unsupervised classification. International Journal of Image and Graphics, 10(01):135-154, 2010.

[125] C. Ciofolo and C. Barillot. Atlas-based segmentation of 3D cerebral structures with competitive level sets and fuzzy control. Medical Image Analysis, 13(3):456-470, 2009.

[126] A. El-Baz et al. Precise segmentation of 3-D magnetic resonance angiography. IEEE Trans Biomed Eng, 59(7):2019-2029, 2012.

[127] A. Farag, A. El-Baz, and G. Gimel'farb. Precise segmentation of multimodal images. IEEE Transaction on Image Processssing, 15(4):952-968, 2006.

[128] P. A. Viola and W. M. Wells III. Alignment by maximization of mutual information. International Journal on Computer Vision, 24(2):137-154, 1997.

[129] J. Besag. On the statistical analysis of dirty pictures. J. Royal Stat. Soc. Series B, 48(3):259-302, 1986.

[130] MICCAI Grand Challenge on MR Brain Image Segmentation (MRBrainS13). http://mrbrains13. isi.uu.nl/workshop. php, September 2013.

[131] A. Alansary, A. Soliman, F. Khalifa, A. Elnakib, M. Mostapha, M. Nitzken, M. Casanova, and A. El-Baz. MAP-based framework for segmentation of $\mathrm{mr}$ brain images based on visual appearance and prior shape. MIDAS Journal [online]. Available: http://hdl.handle.net/10380/3440, October 2013. 
[132] A. Alansary, A. Soliman, M. Nitzken, F. Khalifa, A. Elnakib, M. F. Casanova, and A. El-Baz. An Integrated Geometrical and Stochastic Approach for Accurate Infant Brain Extraction. In Proceedings IEEE International Conference on Image Processing, ICIP-2014, page in press, Paris, France, October 27-30, 2014.

[133] Y. Zhang et al. Segmentation of brain $\mathrm{mr}$ images through a hidden markov random field model and the expectation-maximization algorithm. Medical Imaging, IEEE Transactions on, 20(1):45-57, 2001.

[134] M. F. Casanova, A. Farag, E.-B. Ayman, M. Meghan, H. Hassan, R. Fahmi, and A. E. Switala. Abnormalities of the gyral window in autism: A macroscopic correlate to a putative minicolumnopathy. Journal of Special Education and Rehabilitation, 7(1-2), 2006.

[135] R. Fahmi, A. S. El-Baz, H. Abd El Munim, A. A. Farag, and M. F. Casanova. Classification techniques for autistic vs. typically developing brain using MRI data. In Proc. IEEE International Symposium on Biomedical Imaging: From Nano to Macro (ISBI'2007), pages 1348-1351. IEEE, 2007.

[136] A. A. Farag, R. Fahmi, M. F. Casanova, A. E. Abdel-Hakim, H. A. El-Munim, and A. El-Baz. Robust neuroimaging-based classification techniques of autistic vs. typically developing brain. In Deformable Models, chapter 16, pages 535-566. Springer, 2007.

[137] R. Fahmi, A. Elbaz, H. Hassan, A. A. Farag, and M. F. Casanova. Structural MRI-based discrimination between autistic and typically developing brain. In Proc. Computer Assisted Radiology and Surgery (CARS'2007), pages 24-26, 2007.

[138] A. El-Baz, M. F. Casanova, G. Gimel'farb, M. Mott, and A. E. Switala. Autism diagnostics by $3 \mathrm{D}$ texture analysis of cerebral white matter gyrifications. 
In Proc. International Conference on Medical Image Computing and ComputerAssisted Intervention (MICCAI'2007), pages 882-890. Springer, 2007.

[139] M. F. Casanova, A. El-Baz, M. Mott, G. Mannheim, H. Hassan, R. Fahmi, J. Giedd, J. M. Rumsey, A. E. Switala, and A. Farag. Reduced gyral window and corpus callosum size in autism: Possible macroscopic correlates of a minicolumnopathy. Journal of Autism and Developmental Disorders, 39(5):751$764,2009$.

[140] M. F. Casanova, A. El-Baz, E. Vanbogaert, P. Narahari, and A. Switala. A topographic study of minicolumnar core width by lamina comparison between autistic subjects and controls: Possible minicolumnar disruption due to an anatomical element in-common to multiple laminae. Brain Pathology, 20(2):451-458, 2010.

[141] M. F. Casanova, A. S. El-Baz, S. S. Kamat, B. A. Dombroski, F. Khalifa, A. Elnakib, A. Soliman, A. Allison-McNutt, and A. E. Switala. Focal cortical dysplasias in autism spectrum disorders. Acta Neuropathologica Communications, 1(1):67, 2013.

[142] M. Nitzken, M. Casanova, G. Gimel'farb, A. Elnakib, F. Khalifa, A. Switala, and A. El-Baz. 3d shape analysis of the brain cortex with application to dyslexia. In Image Processing (ICIP), 2011 18th IEEE International Conference on, pages 2657-2660. IEEE, 2011.

[143] E. L. Williams, A. El-Baz, M. Nitzken, A. E. Switala, and M. F. Casanova. Spherical harmonic analysis of cortical complexity in autism and dyslexia. Translational neuroscience, 3(1):36-40, 2012.

[144] S. E. Yuksel, A. El-Baz, A. A. Farag, M. Abo El-Ghar, T. A. Eldiasty, and M. A. Ghoneim. Automatic detection of renal rejection after kidney transplantation. In International Congress Series, volume 1281, pages 773-778, 2005. 
[145] S. E. Yuksel, A. El-Baz, and A. A. Farag. A kidney segmentation framework for dynamic contrast enhanced magnetic resonance imaging. In Proc. International Symposium on Mathematical Methods in Engineering (MME'2006), pages 55-64, Ankara, Turkey, April, 27-29, 2006.

[146] S. E. Yuksel, A. El-Baz, A. A. Farag, M. El-Ghar, T. Eldiasty, and M. A. Ghoneim. A kidney segmentation framework for dynamic contrast enhanced magnetic resonance imaging. Journal of Vibration and Control, 13(910):1505-1516, 2007.

[147] A. M. Ali, A. A. Farag, and A. El-Baz. Graph cuts framework for kidney segmentation with prior shape constraints. In Proc. International Conference on Medical Image Computing and Computer-Assisted Intervention (MICCAI'2007), volume 1, pages 384-392, Brisbane, Australia, October 29-November 2, 2007.

[148] A. El-Baz, A. Farag, R. Fahmi, S. Yuksel, W. Miller, M. A. El-Ghar, T. ElDiasty, and M. Ghoneim. A new CAD system for the evaluation of kidney diseases using DCE-MRI. In Proc. International Conference on Medical Image Computing and Computer-Assisted Intervention (MICCAI'2006), pages 446-453, Copenhagen, Denmark, October 1-6, 2006.

[149] F. Khalifa, G. Gimel'farb, M. A. El-Ghar, G. Sokhadze, S. Manning, P. McClure, R. Ouseph, and A. El-Baz. A new deformable model-based segmentation approach for accurate extraction of the kidney from abdominal CT images. In Proc. International Conference on Image Processing (ICIP'2011), pages 3393-3396, Brussels, Belgium, September 11-14, 2011.

[150] F. Khalifa, A. Elnakib, G. M. Beache, G. Gimel'farb, M. A. El-Ghar, G. Sokhadze, S. Manning, P. McClure, and A. El-Baz. 3D kidney segmentation from CT images using a level set approach guided by a novel stochastic speed function. In Proc. International Conference on Medical Image Computing 
and Computer-Assisted Intervention (MICCAI'2011), pages 587-594, Toronto, Canada, Sept. 18-22, 2011.

[151] F. Khalifa, M. A. El-Ghar, B. Abdollahi, H. Frieboes, T. El-Diasty, and A. ElBaz. A comprehensive non-invasive framework for automated evaluation of acute renal transplant rejection using DCE-MRI. NMR in Biomedicine, 26(11):1460-1470, 2013.

[152] M. Mostapha, F. Khalifa, A. Alansary, A. Soliman, G. Gimel'farb, and A. ElBaz. Dynamic MRI-based computer aided diagnostic systems for early detection of kidney transplant rejection: A survey. In Proc. International Symposium on Computational Models for Life Sciences (CMLS'2013), volume 1559, pages 297-306, 2013.

[153] M. Mostapha, F. Khalifa, A. Alansary, A. Soliman, J. Suri, and A. ElBaz. Computer-aided diagnosis systems for acute renal transplant rejection: Challenges and methodologies. In A. El-Baz and L. saba J. Suri, editors, $A b$ domen and Thoracic Imaging, chapter 1, pages 1-35. Springer, 2014.

[154] A. El-Baz, A. A. Farag, R. Falk, and R. La Rocca. Automatic identification of lung abnormalities in chest spiral CT scans. In Proc. IEEE International Conference on Acoustics, Speech, and Signal Processing, (ICASSP'2003), volume 2, pages II-261. IEEE, 2003.

[155] A. El-Baz, A. A. Farag, R. Falk, and R. La Rocca. A unified approach for detection, visualization, and identification of lung abnormalities in chest spiral CT scans. In International Congress Series, volume 1256, pages 998-1004. Elsevier, 2003.

[156] A. A. Farag, A. El-Baz, G. Gimel'farb, and R. Falk. Detection and recognition of lung abnormalities using deformable templates. In Proc. International 
Conference on Pattern Recognition (ICPR'2004), volume 3, pages 738-741. IEEE, 2004.

[157] A. A. Farag, A. El-Baz, G. G. Gimelfarb, R. Falk, and S. G. Hushek. Automatic detection and recognition of lung abnormalities in helical CT images using deformable templates. In Proc. International Conference on Medical Image Computing and Computer-Assisted Intervention (MICCAI'2004), pages 856-864. Springer, 2004.

[158] A. Farag, A. El-Baz, G. Gimel'farb, and R. Falk. Detection and recognition of lung nodules in spiral CT images using deformable templates and Bayesian post-classification. In Proc. International Conference on Image Processing (ICIP'2004), volume 5, pages 2921-2924. IEEE, 2004.

[159] A. El-Baz, S. E. Yuksel, S. Elshazly, and A. A. Farag. Non-rigid registration techniques for automatic follow-up of lung nodules. In Proc. Computer Assisted Radiology and Surgery (CARS'2005), volume 1281, pages 1115-1120. Elsevier, 2005.

[160] A. A. Farag, A. El-Baz, G. Gimelfarb, M. Abou El-Ghar, and T. Eldiasty. Quantitative nodule detection in low dose chest CT scans: New template modeling and evaluation for CAD system design. In Proc. International Conference on Medical Image Computing and Computer-Assisted Intervention (MICCAI'2005), pages 720-728. Springer, 2005.

[161] A. El-Baz, A. Farag, G. Gimel'farb, R. Falk, M. Abou El-Ghar, and T. Eldiasty. A framework for automatic segmentation of lung nodules from low dose chest CT scans. In Proc. International Conference on Pattern Recognition (ICPR'2006), volume 3, pages 611-614. IEEE, 2006.

[162] A. M. Ali, A. S. El-Baz, and A. A. Farag. A novel framework for accurate lung segmentation using graph cuts. In Proc. IEEE International Symposium 
on Biomedical Imaging: From Nano to Macro (ISBI'2007), pages 908-911. IEEE, 2007.

[163] A. El-Baz, G. Gimel'farb, R. Falk, and M. Abou El-Ghar. A novel approach for automatic follow-up of detected lung nodules. In Proc. International Conference on Image Processing (ICIP'2007), volume 5, pages 501-504. IEEE, 2007.

[164] A. El-Baz, M. F. Casanova, G. Gimel'farb, M. Mott, and A. E. Switwala. A new image analysis approach for automatic classification of autistic brains. In Proc. IEEE International Symposium on Biomedical Imaging: From Nano to Macro (ISBI'2007), pages 352-355. IEEE, 2007.

[165] A. A. Farag, A. El-Baz, G. Gimel'farb, R. Falk, M. A. El-Ghar, T. Eldiasty, and S. Elshazly. Appearance models for robust segmentation of pulmonary nodules in 3D LDCT chest images. In Proc. International Conference on Medical Image Computing and Computer-Assisted Intervention (MICCAI'2006), pages 734-741, Copenhagen, Denmark, October 1-6, 2006.

[166] A. M. Ali and A. A. Farag. Automatic lung segmentation of volumetric lowdose CT scans using graph cuts. In Advances in Visual Computing, pages 258267. Springer, 2008.

[167] A. El-Baz, G. L. Gimel'farb, R. Falk, M. Abou El-Ghar, T. Holland, and T. Shaffer. A new stochastic framework for accurate lung segmentation. In Proc. International Conference on Medical Image Computing and ComputerAssisted Intervention (MICCAI'2008), pages 322-330, 2008.

[168] A. El-Baz, G. L. Gimel'farb, R. Falk, D. Heredis, and M. Abou El-Ghar. A novel approach for accurate estimation of the growth rate of the detected lung nodules. In Proc. International Workshop on Pulmonary Image Analysis, pages 33-42, 2008. 
[169] A. El-Baz, G. L. Gimel'farb, R. Falk, T. Holland, and T. Shaffer. A framework for unsupervised segmentation of lung tissues from low dose computed tomography images. In Proc. British Machine Vision (BMVC'2008), pages 1-10, 2008.

[170] A. El-Baz, G. Gimel'farb, R. Falk, and M. Abou El-Ghar. A new approach for automatic analysis of 3D low dose $\mathrm{CT}$ images for accurate monitoring the detected lung nodules. In Proc. International Conference on Pattern Recognition (ICPR'2008), pages 1-4. IEEE, 2008.

[171] A. El-Baz, G. Gimel'farb, R. Falk, M. Abou El-Ghar, and H. Refaie. Promising results for early diagnosis of lung cancer. In Proc. IEEE International Symposium on Biomedical Imaging: From Nano to Macro (ISBI'2008), pages 1151-1154. IEEE, 2008.

[172] A. El-Baz, G. Gimelfarb, R. Falk, and M. Abo El-Ghar. Automatic analysis of 3D low dose CT images for early diagnosis of lung cancer. Pattern Recognition, 42(6):1041-1051, 2009.

[173] A. El-Baz, G. Gimelfarb, R. Falk, M. Abou El-Ghar, S. Rainey, D. Heredia, and T. Shaffer. Toward early diagnosis of lung cancer. In Proc. International Conference on Medical Image Computing and Computer-Assisted Intervention (MICCAI'2009), pages 682-689. Springer, 2009.

[174] A. El-Baz, G. Gimel'farb, R. Falk, and M. El-Ghar. Appearance analysis for diagnosing malignant lung nodules. In Proc. IEEE International Symposium on Biomedical Imaging: From Nano to Macro (ISBI'2010), pages 193-196. IEEE, 2010.

[175] A. El-Baz, P. Sethu, G. Gimel'farb, F. Khalifa, A. Elnakib, R. Falk, and M. Abo El-Ghar. Elastic phantoms generated by microfluidics technology: Valida- 
tion of an imaged-based approach for accurate measurement of the growth rate of lung nodules. Biotechnology Journal, 6(2):195-203, 2011.

[176] A. El-Baz, G. Gimelfarb, R. Falk, M. Abo El-Ghar, and J. Suri. Appearance analysis for the early assessment of detected lung nodules. In Lung Imaging and Computer Aided Diagnosis, chapter 17, pages 395-404. CRC Press, 2011.

[177] A. El-Baz, P. Sethu, G. Gimelfarb, F. Khalifa, A. Elnakib, R. Falk, A. M. ElGhar, and J. Suri. Validation of a new imaged-based approach for the accurate estimating of the growth rate of detected lung nodules using real computed tomography images and elastic phantoms generated by state-oftheart microfluidics technology. In Lung Imaging and Computer Aided Diagnosis, chapter 18, pages 405-420. CRC Press, 2011.

[178] A. El-Baz, M. Nitzken, G. Gimelfarb, E. Van Bogaert, R. Falk, M. Abo El-Ghar, and J. Suri. Three-dimensional shape analysis using spherical harmonics for early assessment of detected lung nodules. In Lung Imaging and Computer Aided Diagnosis, chapter 19, pages 421-438. CRC Press, 2011.

[179] B. Abdollahi, A. Soliman, A. Civelek, X.-F. Li, G. Gimel'farb, and A. ElBaz. A novel gaussian scale space-based joint MGRF framework for precise lung segmentation. In Proc. International Conference on Image Processing (ICIP'2012), pages 2029-2032. IEEE, 2012.

[180] A. El-Baz, F. Khalifa, A. Elnakib, M. Nitzken, A. Soliman, P. McClure, M. Abou El-Ghar, and G. Gimelfarb. A novel approach for global lung registration using 3D Markov-Gibbs appearance model. In Proc. International Conference on Medical Image Computing and Computer-Assisted Intervention (MICCAI'2012), pages 114-121. Springer, 2012.

[181] A. El-Baz, G. Gimel'farb, M. Abou El-Ghar, and R. Falk. Appearance-based diagnostic system for early assessment of malignant lung nodules. In Proc. 
International Conference on Image Processing (ICIP'2012), pages 533-536. IEEE, 2012.

[182] A. El-Baz, A. Soliman, P. McClure, G. Gimel'farb, M. Abou El-Ghar, and R. Falk. Early assessment of malignant lung nodules based on the spatial analysis of detected lung nodules. In Proc. IEEE International Symposium on Biomedical Imaging: From Nano to Macro (ISBI'2012), pages 1463-1466. IEEE, 2012.

[183] A. Soliman, F. Khalifa, A. Alansary, G. Gimel'farb, and A. El-Baz. Segmentation of lung region based on using parallel implementation of joint MGRF: Validation on 3D realistic lung phantoms. In Proc. IEEE International Symposium on Biomedical Imaging: From Nano to Macro (ISBI'2013), pages 852-855. IEEE, 2013.

[184] A. El-Baz, A. Elnakib, M. Abou El-Ghar, G. Gimel'farb, R. Falk, and A. Farag. Automatic detection of 2D and 3D lung nodules in chest spiral CT scans. International Journal of Biomedical Imaging, 2013, 2013.

[185] A. El-Baz, G. M. Beache, G. Gimel'farb, K. Suzuki, K. Okada, A. Elnakib, A. Soliman, and B. Abdollahi. Computer-aided diagnosis systems for lung cancer: Challenges and methodologies. International Journal of Biomedical Imaging, 2013, 2013.

[186] A. El-Baz, M. Nitzken, F. Khalifa, A. Elnakib, G. Gimelfarb, R. Falk, and M. Abo El-Ghar. 3D shape analysis for early diagnosis of malignant lung nodules. In Proc. Information Processing in Medical Imaging, (IPMI'2011), pages 772-783. Springer, 2011.

[187] A. Soliman, F. Khalifa, A. Alansary, G. Gimel'farb, and A. El-Baz. Performance evaluation of an automatic MGRF-based lung segmentation ap- 
proach. In Proc. International Symposium on Computational Models for Life Sciences (CMLS'2013), volume 1559, pages 323-332, 2013.

[188] A. Firjany, A. Elnakib, A. El-Baz, G. Gimel'farb, M. A. El-Ghar, and A. Elmagharby. Novel stochastic framework for accurate segmentation of prostate in dynamic contrast enhanced MRI. In Prostate Cancer Imaging. Computer-Aided Diagnosis, Prognosis, and Intervention, pages 121-130. Springer, 2010.

[189] A. Firjani, A. Elnakib, F. Khalifa, A. El-Baz, G. Gimel'farb, M. A. El-Ghar, and A. Elmaghraby. A novel 3D segmentation approach for segmenting the prostate from dynamic contrast enhanced MRI using current appearance and learned shape prior. In Proc. IEEE International Symposium on Signal Processing and Information Technology (ISSPIT'2010), pages 137-143. IEEE, 2010.

[190] A. Firjani, A. Elnakib, F. Khalifa, G. Gimel'farb, M. A. El-Ghar, J. Suri, A. Elmaghraby, and A. El-Baz. A new 3D automatic segmentation framework for accurate segmentation of prostate from DCE-MRI. In Proc. IEEE International Symposium on Biomedical Imaging: From Nano to Macro (ISIB'2011), pages 1476-1479. IEEE, 2011.

[191] A. Firjani, F. Khalifa, A. Elnakib, G. Gimel'farb, M. A. El-Ghar, A. Elmaghraby, and A. El-Baz. 3D automatic approach for precise segmentation of the prostate from diffusion-weighted magnetic resonance imaging. In Proc. IEEE International Conference on Image Processing (ICIP'2011), pages 2285-2288. IEEE, 2011.

[192] A. Firjani, A. Elnakib, F. Khalifa, G. Gimel'farb, M. A. El-Ghar, A. Elmaghraby, and A. El-Baz. A new 3D automatic segmentation framework for accurate extraction of prostate from diffusion imaging. In Proc. Biomedical Sciences and Engineering Conference (BSEC'2011), pages 1-4. IEEE, 2011. 
[193] A. Firjani, A. Elnakib, F. Khalifa, G. Gimel'farb, M. A. El-Ghar, A. Elmaghraby, and A. El-Baz. A diffusion-weighted imaging based diagnostic system for early detection of prostate cancer. Journal of Biomedical Science and Engineering (JBiSE), 6:346-356, 2013.

[194] A. Firjani, F. Khalifa, A. Elnakib, G. Gimel'farb, M. A. El-Ghar, A. Elmaghraby, and A. El-Baz. A novel image-based approach for early detection of prostate cancer using DCE-MRI. In K. Suzuki, editor, Computational Intelligence in Biomedical Imaging, chapter 3, pages 55-85. Springer Science and Business Media, 2014.

[195] R. C. Dubes and A. K. Jain. Random field models in image analysis. Journal of Applied Statistics, 16(2):131-164, 1989.

[196] G. L. Gimel'farb and A. Zalesny. Probabilistic models of digital region maps based on Markov random fields with short-and long-range interaction. Pattern Recognition Letters, 14(10):789-797, 1993.

[197] R. W. Picard and I. M. Elfadel. Structure of aura and co-occurrence matrices for the Gibbs texture model. Journal of Mathematical Imaging and Vision, 2(1):5-25, 1992.

[198] I. L. Dryden, M. R. Scarr, and C. C. Taylor. Bayesian texture segmentation of weed and crop images using reversible jump Markov chain Monte Carlo methods. Journal of the Royal Statistical Society: Series C (Applied Statistics), 52(1):31-50, 2003.

[199] G. L. Gimel'farb. Image textures and Gibbs random fields, volume 16. Springer, 1999.

[200] J. Besag. Spatial interaction and the statistical analysis of lattice systems. Journal of the Royal Statistical Society. Series B (Methodological), pages 192-236, 1974. 


\section{APPENDICES}

\section{A Appendix I - Analytical Estimation of the bi-valued Gibbs Potentials}

Let $\mathbf{Q}=\{0, \ldots, Q-1\}$ and $\mathbf{L}=\{0, \ldots, L-1\}$ denote sets of gray levels $q$ and region labels $k$, respectively. Here, $Q$ is the number of gray levels and $K$ is the number of image modes, i.e. peaks in the gray level frequency distribution, e.g., for a bimodal image, $L=2$. We assume that each dominant image mode corresponds to a particular class of objects to be found in the image.

Let $\mathbf{R}=\{(x, y, z): 0 \leq x \leq X-1,0 \leq y \leq Y-1,0 \leq z \leq Z-1\}$ be a 3D $(x, y, z)$-arithmetic grid supporting gray level images $\mathbf{g}: \mathbf{R} \rightarrow \mathbf{Q}$ and their region maps $\mathbf{m}: \mathbf{R} \rightarrow \mathbf{L}$. A two-level probability model of original images to segment and their desired region maps is given by a joint distribution $P(\mathbf{g}, \mathbf{m})=P(\mathbf{m}) P(\mathbf{g} \mid \mathbf{m})$ where $P(\mathbf{m})$ is an unconditional probability distribution of maps $\left(2^{\text {nd }}\right.$-order spatial Markov Gibbs random field (MGRF) model) and $P(\mathbf{g} \mid \mathbf{m})$ is a conditional distribution of images, given the map ( $1^{\text {st }}$-order intensity model). The Bayesian maximum a posteriori (MAP) estimate of the map $\mathbf{m}$, given the image $\mathbf{g}$ :

$$
\mathbf{m}^{*}=\arg \max _{\mathbf{m} \in \mathcal{X}} L(\mathbf{g}, \mathbf{m})
$$

where $\mathcal{X}$ is the set of all region maps with labels $\lambda \in \mathbf{L}$ on $\mathbf{R}$, maximizes the loglikelihood function:

$$
L(\mathbf{g}, \mathbf{m})=\frac{1}{|\mathbf{R}|}(\log P(\mathbf{g} \mid \mathbf{m})+\log P(\mathbf{m}))
$$

To find this estimate, we need to select the $1^{\text {st }}$-order intensity model and $2^{\text {nd }}$-order spatial MGRF model and identify their parameters.

\section{Unconditional Region Map Model}

The simplest model of interdependent region labels is the MGRF with the nearest 26-neighborhood of each voxel. By symmetry considerations, we assume 
the Gibbs potentials are independent of relative orientation of voxel pairs, are the same for all classes, and depend only on whether the pair of labels are equal or not. Under these assumptions, it is the simplest auto-binomial model, the Potts one, being for a long time a popular region map model [195-198]. But unlike the conventional counterparts, its Gibbs potential is obtained analytically using the maximum likelihood estimator for a generic MGRF derived in [199]. The 26neighborhood results in a family $\mathbf{C}_{\mathbf{N}}=\left[\mathbf{c}_{x, y, z, \xi, \eta, \kappa}=((x, y, z),(x+\xi, y+\eta, z+\kappa))\right.$ : $\left.(x, y, z) \in \mathbf{R} ;(x+\xi, y+\eta, z+\kappa) \in \mathbf{R} ;(\xi, \eta, \kappa) \in \nu_{s}\right]$ of the neighboring voxel pairs supporting the Gibbs potentials. The potentials are bi-valued because only the coincidence of the labels is taken into account: $V\left(\lambda, \lambda^{\prime}\right)=V_{\text {eq }}$ if $\lambda^{\prime}=\lambda^{\prime}$ and $V_{\text {ne }}$ if $\lambda \neq \lambda^{\prime}$. Then the MGRF model of region maps is as follows:

$$
\begin{aligned}
P(\mathbf{m}) & =\frac{1}{Z_{\mathbf{N}}} \exp \sum_{(x, y, z) \in \mathbf{R}} \sum_{(\xi, \eta, \kappa) \in \nu_{s}} V\left(m_{x, y, z}, m_{x+\xi, y+\eta, z+\kappa}\right) \\
& =\frac{1}{Z_{\mathbf{N}}} \exp \left(\left|\mathbf{C}_{\mathbf{N}}\right| V_{\text {eq }}\left(2 f_{\text {eq }}(\mathbf{m})-1\right)\right)
\end{aligned}
$$

where $\left|\mathbf{C}_{\mathbf{N}}\right|$ is the cardinality of the family $\mathbf{C}_{\mathbf{N}}$ and $f_{\mathrm{eq}}(\mathbf{m})$ denotes the relative frequency of the equal labels in the voxel pairs of this family:

$$
f_{\mathrm{eq}}(\mathbf{m})=\frac{1}{\left|\mathbf{C}_{\mathbf{N}}\right|} \sum_{\mathbf{c}_{x, y, z, \xi, \eta, \kappa} \in \mathbf{C}_{\mathbf{N}}} \delta\left(m_{x, y, z}-m_{x+\xi, y+\eta, z+\kappa}\right)
$$

Here, $\delta()$ denotes the Kronecker delta function: $\delta(0)=1$ and 0 otherwise. To identify the $2^{\text {nd }}$-order MGRF model, we have to estimate only the potential value $V_{\text {eq }}$.

To compute the second term, $\frac{1}{|\mathbf{R}|} \log P(\mathbf{m})$, in Eq. (16) for a region map $\mathbf{m}$, we use the approximate partition function $Z_{N}$ in [200] (see also [195], p.156) reduced 
in our case to:

$$
\begin{aligned}
Z_{\mathbf{N}} & \approx \exp \left(\sum_{x, y, z \in \mathbf{R}} \sum_{\xi, \eta, \kappa \in \nu_{s}} \sum_{\lambda \in \mathbf{L}} V\left(\lambda, m_{x+\xi, y+\eta, z+\kappa}\right)\right) \\
& =\exp \left(\left|\mathbf{C}_{\mathbf{N}}\right| \sum_{\lambda \in \mathbf{L}}\left(V_{\mathrm{eq}} f_{\lambda}(\mathbf{m})-V_{\mathrm{eq}}\left(1-f_{\lambda}(\mathbf{m})\right)\right)\right) \\
& =\exp \left(V_{\mathrm{eq}}\left|\mathbf{C}_{\mathbf{N}}\right|(2-L)\right)
\end{aligned}
$$

where $f_{\lambda}(\mathbf{m})$ is the marginal frequency of the label $\lambda$ in the map $\mathbf{m}$. The above approximate partition function (which becomes too trivial for $L=2$ ) results in the following approximation of the second term $\frac{1}{|\mathbf{R}|} \log P(\mathbf{m})$ in Eq. (16):

$$
\varrho V_{\mathrm{eq}}\left(2 f_{\mathrm{eq}}(\mathbf{m})+L-3\right) \approx 4 V_{\mathrm{eq}}\left(2 f_{\mathrm{eq}}(\mathbf{m})+L-3\right)
$$

where $\varrho=\frac{\left|\mathbf{C}_{\mathbf{N}}\right|}{|\mathbf{R}|} \approx\left|\nu_{s}\right|=4$.

\section{Identification of the $2^{\text {nd }}$-order MGRF model}

The approximate log-likelihood term in Eq. (19) is unsuitable for estimating the model parameter $V_{\text {eq }}$ that specifies the Gibbs potential. Thus we identify the $2^{\text {nd }}$-order MGRF model using a reasonably close first approximation of the maximum likelihood estimate (MLE) of $V_{\text {eq }}$ derived for a given region map $\mathbf{m}^{\circ}$ in accord with [199] from the unconditional log-likelihood $L_{u}\left(\mathbf{m}^{\circ} \mid V_{\text {eq }}\right)=\frac{1}{|\mathbf{R}|} \log P\left(\mathbf{m}^{\circ}\right)$ of Eq. (17) with the exact partition function $Z_{\mathbf{N}}=\sum_{\mathbf{m} \in \mathcal{X}} \exp \left(V_{\text {eq }} \varrho|\mathbf{R}|\left(2 f_{\text {eq }}(\mathbf{m})-1\right)\right)$ where $\mathcal{X}$ is the parent population of region maps:

$$
\begin{aligned}
L_{u}\left(\mathbf{m}^{\circ} \mid V_{\text {eq }}\right) & =V_{\text {eq }} \varrho\left(2 f_{\text {eq }}\left(\mathbf{m}^{\circ}\right)-1\right) \\
& -\frac{1}{|\mathbf{R}|} \log \left(\sum_{\mathbf{m} \in \mathcal{X}} \exp \left(V_{\text {eq }} \varrho|\mathbf{R}|\left(2 f_{\text {eq }}(\mathbf{m})-1\right)\right)\right)
\end{aligned}
$$

The approximation is obtained by truncating the Taylor's series expansion of 
$L\left(\mathbf{m}^{\circ} \mid V_{\mathrm{eq}}\right)$ in the close vicinity of zero potential, $V_{\mathrm{eq}}=0$, to the first three terms:

$$
L_{u}\left(\mathbf{m}^{\circ} \mid 0\right)+\left.V_{\text {eq }} \frac{d L\left(\mathbf{m}^{\circ} \mid V_{\text {eq }}\right)}{d V_{\text {eq }}}\right|_{V_{\text {eq }}=0}+\left.\frac{1}{2} V_{\text {eq }}^{2} \frac{d^{2} L_{u}\left(\mathbf{m}^{\circ} \mid V_{\text {eq }}\right)}{d V_{\text {eq }}^{2}}\right|_{V_{\text {eq }}=0}
$$

Because zero potential produces an independent random field (IRF) equiprobable region labels $\lambda \in \mathbf{L}$, the relative frequency of the equal pairs of labels over $\mathbf{C}_{\mathbf{N}}$ has in this case the mean value $\frac{1}{L}$ and the variance $\frac{L-1}{L^{2}}$. Then the following relationships hold:

$$
\begin{aligned}
& \left.\frac{d L_{u}\left(\mathbf{m}^{\circ} \mid V_{\text {eq }}\right)}{d V_{\text {eq }}}\right|_{V_{\text {eq }}=0}=2 \varrho\left(f_{e q}\left(\mathbf{m}^{\circ}\right)-\frac{1}{L}\right) \\
& \left.\frac{d^{2} L E_{u}\left(\mathbf{m}^{\circ} \mid V_{\text {eq }}\right)}{d V_{\text {eq }}^{2}}\right|_{V_{\text {eq }}=0}=-4 \varrho \frac{L-1}{L^{2}}
\end{aligned}
$$

where $f_{e q}(\mathbf{m})^{\circ}$ is the relative frequency of the equal label pairs in the region map $\mathbf{m}^{\circ}$ specified in Eq. (18). The approximate likelihood of Eq. (20) results in the following MLE of $V_{\text {eq }}$ for a given map $\mathbf{m}^{\circ}$ :

$$
V_{\mathrm{eq}}=\frac{L^{2}}{2(L-1)}\left(f_{\mathrm{eq}}\left(\mathbf{m}^{\circ}\right)-\frac{1}{L}\right)
$$

This relationship allows for computing the potentials of the Potts model for each current region map obtained by the Bayesian classification based on the estimated low-level image model. For bimodal images $(L=2)$, the value $V_{\text {eq }}$ is estimated as:

$$
V_{\text {eq }}=2 f_{\text {eq }}\left(\mathbf{m}^{\circ}\right)-1
$$

\section{B Appendix II - Analytical Estimation of Gibbs Potentials for Higher-Order MGRF Model}

Let $\mathbf{L}=\{0, \ldots, L\}$ and $\mathbf{R}=\{(x, y, z): 0 \leq x \leq X-1,0 \leq y \leq Y-1,0 \leq$ $z \leq Z-1$,$\} denote a set of region labels L$ and a finite arithmetic lattice supporting binary maps $\mathbf{m}: \mathbf{R} \rightarrow \mathbf{L}$, respectively. Let $\mathbf{C}_{a}$ be a family of $s$-order cliques of interaction graph with nodes in the lattice sites $(x, y, z)$ and edges connecting the interacting (interdependent) sites. Let $A$ clique families describe the spatial 
geometry of interactions between the region labels of region maps for an MGRF model:

$$
P_{\mathbf{V}}(\mathbf{m})=\frac{1}{Z_{\mathbf{V}}} \exp \left(\sum_{a=1}^{A} \sum_{\mathbf{c} \in \mathbf{C}_{a}} V_{a}(\mathbf{m}(x, y, z):(x, y, z) \in \mathbf{c})\right)
$$

where $\mathbf{V}=\left[V_{a}:\{0, \ldots, L\} \rightarrow(-\infty, \infty): a=1, \ldots, A\right]$ is a collection of potential functions for the families $\mathbf{C}_{a}$ and $Z_{\mathbf{V}}$ is the partition function

$$
Z_{\mathbf{V}}=\sum_{\mathbf{m} \in \mathbb{M}} \exp \left(\sum_{a=1}^{A} \sum_{\mathbf{c} \in \mathbf{C}_{a}} V_{a}(\mathbf{m}(x, y, z):(x, y, z) \in \mathbf{c})\right)
$$

normalising the probabilities over the population $\mathbb{M}=\{0, \ldots, L\}^{X Y Z}$ of the maps. Let $\mathbf{F}\left(\mathbf{m}^{\circ}\right)=\left[\rho_{a} F_{a}\left(\mu_{1}, \ldots, \mu_{s} \mid \mathbf{m}^{\circ}\right):\left(\mu_{1}, \ldots, \mu_{s}\right) \in\{0, \ldots, L\}^{s} ; a=1, \ldots, A\right]$ and $\mathbf{P}_{\mathbf{V}}=\left[\rho_{a} P_{a}\left(\mu_{1}, \ldots, \mu_{s} \mid \mathbf{V}\right):\left(\mu_{1}, \ldots, \mu_{s}\right) \in\{0, \ldots, L\}^{s} ; a=1, \ldots, A\right]$

where $\rho_{a}=\frac{1}{X Y Z}\left|\mathbf{C}_{a}\right|$ is the relative cardinality of the clique family with respect to the lattice cardinality, denote the collection of scaled relative frequencies $F_{a}\left(\mu_{1}, \ldots, \mu_{s} \mid \mathbf{m}^{\circ}\right)$ of co-occurrences of configurations

$\left(\mu_{1}, \ldots, \mu_{s}\right)$ of the labels in the cliques of each family $\mathbf{C}_{a}$ over a given training map $\mathbf{m}^{\circ}$ and the corresponding scaled marginal probabilities $P_{a}\left(\mu_{1}, \ldots, \mu_{s} \mid \mathbf{V}\right)$ of these configurations for the MGRF model, respectively.

The normalized $\log$-likelihood $L\left(\mathbf{V} \mid \mathbf{m}^{\circ}\right)=\frac{1}{X Y Z} \log \mathbf{P}_{\mathbf{V}}\left(\mathbf{m}^{\circ}\right)$ of the map $\mathbf{m}^{\circ}$ has the gradient

$$
\nabla L\left(\mathbf{V} \mid \mathbf{m}^{\circ}\right) \equiv \frac{\partial}{\partial \mathbf{V}} L\left(\mathbf{V} \mid \mathbf{m}^{\circ}\right)=\mathbf{F}\left(\mathbf{m}^{\circ}\right)-\mathbf{P}_{\mathbf{V}}
$$

and its Hessian matrix of the second derivatives is equal to the negated covariance matrix of the marginal probabilities of signal co-occurrences in the cliques (due to non-negative definiteness of the covariance matrix, the log-likelihood is unimodal over the potential space).

The analytical potential estimate (i.e., the approximate maximum likelihood estimate) is specified as scaled gradient vector at the origin in the potential space: 


$$
\mathbf{V}^{*}=\lambda^{*}\left(\mathbf{F}\left(\mathbf{m}^{\circ}\right)-\mathbf{P}_{\mathbf{0}}\right)
$$

where the factor $\lambda^{*}$ maximises the truncated (to the first three terms) Taylor series decomposition of the log-likelihood

$L\left(\mathbf{V} \mid \mathbf{m}^{\circ}\right)$ about the origin:

$$
\begin{aligned}
L\left(\mathbf{V} \mid \mathbf{m}^{\circ}\right) \approx & L\left(\mathbf{0} \mid \mathbf{m}^{\circ}\right)+\lambda\left(\mathbf{F}\left(\mathbf{m}^{\circ}\right)-\mathbf{P}_{\mathbf{0}}\right)^{\top}\left(\mathbf{F}\left(\mathbf{m}^{\circ}\right)-\mathbf{P}_{\mathbf{0}}\right) \\
& -\frac{\lambda^{2}}{2}\left(\mathbf{F}\left(\mathbf{m}^{\circ}\right)-\mathbf{P}_{\mathbf{0}}\right)^{\top} \mathbf{D}_{\mathbf{0}}\left(\mathbf{F}\left(\mathbf{m}^{\circ}\right)-\mathbf{P}_{\mathbf{0}}\right)
\end{aligned}
$$

where $\mathbf{D}_{0}$ denotes the covariance matrix for marginal clique-wise probabilities at the origin. Generally,

$$
\lambda^{*}=\frac{\left(\mathbf{F}\left(\mathbf{m}^{\circ}\right)-\mathbf{P}_{\mathbf{0}}\right)^{\top}\left(\mathbf{F}\left(\mathbf{m}^{\circ}\right)-\mathbf{P}_{\mathbf{0}}\right)}{\left(\mathbf{F}\left(\mathbf{m}^{\circ}\right)-\mathbf{P}_{\mathbf{0}}\right)^{\top} \mathbf{D}_{\mathbf{0}}\left(\mathbf{F}\left(\mathbf{m}^{\circ}\right)-\mathbf{P}_{\mathbf{0}}\right)}
$$

The origin, $\mathbf{V}=\mathbf{0}$ (zero potentials), corresponds to an independent random field (IRF) of equiprobable labels, so that the covariance matrix is closely approximated with the diagonal matrix of variances.

For the labels, $m(x, y, z) \in\{0, \ldots, L\}$, the marginal co-occurrence probabilities over cliques of second, third, and fourth order are $\frac{1}{4}, \frac{1}{8}$, and $\frac{1}{16}$, respectively. If for the symmetry sake, only equality and inequality of the labels are taken into account, then the combinations of co-occurrences and their probabilities are summarized in Table 5.

Provided the cardinalities of the clique families are close to the lattice cardinality (so that $\rho_{a} \approx 1$ ) for all the families $a=1, \ldots, A$, the resulting potential estimates for the second- and third-order models are independent of the number $A$ of the clique families:

Second-order: $V_{2: a: \text { eq }}=-V_{2: a: \text { ne }}=4\left(F_{a: \text { eq }}\left(\mathbf{m}^{\circ}\right)-\frac{1}{2}\right)$

Third-order: $V_{3: a: \mathrm{eq}_{3}}=-V_{3: a: \mathrm{eq}_{2}}=\frac{16}{3}\left(F_{a: \mathrm{eq}_{3}}\left(\mathbf{m}^{\circ}\right)-\frac{1}{4}\right)$ 
TABLE 5. Label combinations and their marginal probabilities for second-, third-, and fourth-order cliques. Here, $p$ denote the probability, "eq" and "ne" denote two equal or non-equal labels for a second-order clique, and "eq $i$ " denote $i$ equal labels for a third- or fourth-order clique.

\begin{tabular}{|l|c|c|c|c|c|c|c|}
\hline Clique order & \multicolumn{2}{|c|}{2} & \multicolumn{2}{c|}{3} & \multicolumn{3}{c|}{4} \\
\hline Label combinations & $\mathrm{eq}$ & ne & $\mathrm{eq}_{3}$ & $\mathrm{eq}_{2}$ & $\mathrm{eq}_{4}$ & $\mathrm{eq}_{3}$ & $\mathrm{eq}_{2}$ \\
\hline Marginal $p$ for the IRF & $1 / 2$ & $1 / 2$ & $1 / 4$ & $3 / 4$ & $1 / 8$ & $1 / 2$ & $3 / 8$ \\
\hline Its variance $p(1-p)$ & $1 / 4$ & $1 / 4$ & $3 / 16$ & $3 / 16$ & $7 / 64$ & $1 / 4$ & $15 / 64$ \\
\hline
\end{tabular}

But for the fourth-order model the factor depends on the number of clique families and the marginal probabilities of label combinations for these families on the training map $\mathbf{m}^{\circ}$ :

$$
\begin{aligned}
& V_{4: a: \mathrm{eq}_{4}}=\lambda^{*}\left(F_{a: \mathrm{eq}_{4}}\left(\mathbf{m}^{\circ}\right)-\frac{1}{8}\right) \\
& V_{4: a: \mathrm{eq}_{3}}=\lambda^{*}\left(F_{a: \mathrm{eq}_{3}}\left(\mathbf{m}^{\circ}\right)-\frac{1}{2}\right) \\
& V_{4: a: \mathrm{eq}_{2}}=\lambda^{*}\left(F_{a: \mathrm{eq}_{2}}\left(\mathbf{m}^{\circ}\right)-\frac{3}{8}\right)=-\left(V_{4: a: \mathrm{eq}_{4}}+V_{4: a: \mathrm{eq}_{3}}\right)
\end{aligned}
$$

where

$$
\lambda^{*}=\frac{\sum_{a=1}^{A}\left(\left(F_{a: \mathrm{eq}_{4}}\left(\mathbf{m}^{\circ}\right)-\frac{1}{8}\right)^{2}+\left(F_{a: \mathrm{eq}_{3}}\left(\mathbf{m}^{\circ}\right)-\frac{1}{2}\right)^{2}+\left(F_{a: \mathrm{eq}_{2}}\left(\mathbf{m}^{\circ}\right)-\frac{3}{8}\right)^{2}\right)}{\sum_{a=1}^{A}\left(\frac{7}{64}\left(F_{a: \mathrm{eq}_{4}}\left(\mathbf{m}^{\circ}\right)-\frac{1}{8}\right)^{2}+\frac{1}{4}\left(F_{a: \mathrm{eq}_{3}}\left(\mathbf{m}^{\circ}\right)-\frac{1}{2}\right)^{2}+\frac{15}{64}\left(F_{a: \mathrm{eq}_{2}}\left(\mathbf{m}^{\circ}\right)-\frac{3}{8}\right)^{2}\right)}
$$




\section{CURRICULUM VITAE}

\section{Amir M. Alansary}

Graduate Student, Research Assistant

Electrical and Computer Engineering Department,

Speed School of Engineering,

University of Louisville

Louisville, Kentucky, USA

\section{Contact Information}

Address: Lutz Hall, University of Louisville Room 304,

Louisville, KY, USA 40292

Phone: +1 (502) 852-4032

E-Mail: amalan01@louisville.edu amiralansary@gmail.com

\section{Personal Statement}

I am a graduate student in the department of Electrical and Computer Engineering at University of Louisville, USA. My general interests are in Digital Image Processing, Medical Imaging, and Computer Vision with a focus on MR brain Images Analysis and my primary expertise is in 3D Probabilistic Atlas Implementation and 3D Segmentation Algorithm. Additionally, I have spent a great many years working in the development of computer programming and have a wide experience in programming a different variety of languages on an array of platforms.

\section{Education and Qualifications}

\section{2-2014 MSc. Electrical and Computer Engineering,} University of Louisville, KY, USA

Have enrolled in different courses and have achieved Excellent levels of performance.

The expected graduation date is August, 2014, with GPA is 4.0/4.0

2004-2009 BSc. Electronics and Communications Engineering, Mansoura University, Egypt 
Achieved Excellent with Honors, ranked 3rd out of 250.

Final Year Project (Group Work): Eye Mouse

A tracking system for eyes and gaze using a webcam to determine location of user's iris in relation to the rest of the eye, using this location to position a computer cursor, programmed in $\mathrm{C}++/$ OpenCV.

Sponsor: Intel cooperation - Egypt

\section{Work Experience}

\section{2-2014 Research Assistant, BioImaging Lab, BioEngineering Department} University of Louisville, Louisville, KY, USA

Projects:

[1] Diagnosing Autism

Designed and Implemented a complete system for segmenting Brain Tissue from infant MR brain Images, programmed in C++/MatLab.

[2] MR Brain Images Tissues Segmentation

Designed and Implemented a complete system to segment the different brain tissues white matter, gray matter, and cerebrospinal fluid using a MAP-based technique for MR brain Images based on visual appearance and prior shape, programmed in $\mathrm{C}++/$ MatLab.

[3] Segmentation of Infant DTI MR Brain Images

Designed and Implemented a complete system to segment the brain tissue from DTI MR Brain Images using an Atlas--Based Approach, programmed in $\mathrm{C}++/$ MatLab

\section{9-2012 Teaching Assistant}

Electronics and Communications Engineering Dept.

Mansoura University, Egypt

Assisted students in their classes and projects, and evaluated course work for Digital Signal Processing, Signal and Systems, Wireless Communications, Digital Image Processing, Programming with MATLAB for Engineers, and Computer Networks 


\section{0-2011 Image Processing Application Developer}

Bookworm (Start Up Company), Mansoura, Egypt

Participated in the design and implementation of a virtual library. Used a webcam to design a user-friendly software to provide an easy, time saving, and entertaining way to read books with a few hand moves, programmed in $\mathrm{C}++/ \mathrm{OpenCV}$

2009-2010 Image Processing Application Developer

Middle East Technology, Cairo, Egypt

Participated in the design and implementation of a virtual changing room. Used an HD webcam to design a user-friendly software to enable customers try on many models with a few hand moves, programmed in $\mathrm{C}++/ \mathrm{OpenCV}$

\section{Prizes and Awards}

2014 [1] "Theobald Scholarship Award" in recognition of valuable contributions to Department of Electrical and Computer Engineering, J.B. Speed School of Engineering, University of Louisville, April 22, 2014.

2013 [2] Won the first prize in the MICCAI Grand Challenge on MR Brain Image Segmentation (MRBrainS13) in Nagoya, Japan in September 2013. For developing a "MAP-Based Framework for Segmentation of MR Brain Images Based on Visual Appearance and Prior Shape”.

2011 [3] Startup summer camp finals, Cairo, Egypt.

2010 [4] Young Innovators Award (YIA) for engineering final year projects.

2009 [5] Won the $5^{\text {th }}$ rank of MIE (Made in Egypt) competition for graduations projects.

[6] Won the first prize of MIE (Made in Egypt) Ethics completion for engineering projects.

[7] Qualified for round-2, Microsoft Imagine Cup for Embedded Development.

2006 [8] Won the prize of the first three candidates at the faculty of Engineering (for academic year 2005) of Higher Education Enhancement Project Fund (HEEPF).

\section{Publications (Conference Papers, Journal Papers, and Book Chapters)}

2014 [1] A. Alansary, A. Soliman, M. Nitzken, F. Khalifa, A. Elnakib, M. F. Casanova, 
and A. El-Baz, "An Integrated Geometrical and Stochastic Approach for Accurate Infant Brain Extraction,” In: Proc. IEEE International Conference on Image Processing (ICIP'14), Paris, France, October 27-30, 2014.

[2] M. Mostapha, A. Alansary, A. Soliman, F. Khalifa, M. Nitzken, M. Casanova, A. El-baz. “Atlas--Based Approach for The Segmentation of Infant DTI MR Brain Images," In: Proc. IEEE International Symposium on Biomedical Imaging: From Nano to Macro (ISBI'14), Bejing, China, April 28 - May 2, 2014.

2013 [3] A. Alansary, A. Soliman, F. Khalifa, A. Elnakib, M. Mostapha, M. Nitzken, M. Casanova, A. El-Baz "MAP-Based Framework for Segmentation of MR Brain Images Based on Visual Appearance and Prior Shape," 2013 Oct. In: MIDAS Journal [online]. Available: http://hdl.handle.net/10380/3440

[4] M. Mostapha, F. Khalifa, A. Alansary, A. Soliman, G. J. Suri, and A. ElBaz, "Computer Aided Diagnosis Systems for Acute Renal Transplant Rejection: Challenges and Methodologies," In: Abdomen and Thoracic Imaging, pp. 1-35, Springer US, 2014.

[5] A. Soliman, F. Khalifa, A. Alansary, G. Gimel'farb and A. El-Baz, "Performance evaluation of an automatic MGRF-based lung segmentation approach," International Symposium on Computational Models for Life Science, Sydney, Australia, vol. 1559, no. 1, pp. 323-332, November 27-29, 2013.

[6] M. Mostapha, F. Khalifa, A. Alansary, A. Soliman, G. Gimel’farb, and A. ElBaz, "Dynamic MRI-Based Computer Aided Diagnostic System for Early Detection of Kidney Transplant Rejection: A Survey," International Symposium on Computational Models for Life Science, Sydney, Australia, vol. 1559, no. 1, pp. 297-306, November 27-29, 2013.

[7] A. Soliman, F. Khalifa, A. Alansary, G. Gimel'farb and A. El-Baz, "Segmentation of Lung Region Based on Using Parallel Implementation of Joint MGRF: Validation on 3D Realistic Lung Phantoms," In: Proc. IEEE International Symposium on Biomedical Imaging: From Nano to Macro (ISBI'13), pp. 852-855, San Francisco, USA, April 7-11, 2013. 


\section{Peer-reviewed Presentations and Challenges}

2013 [1] A. El-Baz, M. Nitzken, A. Soliman, A. Alansary, M. Mostapha, "Tutorial on Stochastic Modeling for Medical Image Analysis," Proc. of International Conference on Medical Image Computing and Computer-Assisted Intervention (MICCAI'13), Nagoya, Japan, September 22-26.

https://louisville.edu/speed/bioengineering/faculty/bioengineering-full/drayman-el-baz/miccai-tutorial.html

[2] A. Alansary, "MICCAI Grand Challenge on MR Brain Image Segmentation (MRBrainS13)," Proc. of International Conference on Medical Image Computing and Computer-Assisted Intervention (MICCAI'13), Nagoya, Japan, September 2226. http://hdl.handle.net/10380/3440

\section{Patents \& Disclosures}

2013 [1] A. El-Baz, A. Soliman, A. Alansary, M. Nitzken, M. Casanova, "Brain Segmentation Method for Young Children and Adults," US Provisional Patent Application \#13100.

\section{Professional Activity}

2013-2014 MICCAI Society Member

2013-2014 IEEE Signal Society Member

2011-2014 IEEE Student Member

2011-2012 IEEE Student’s Mentor, IEEE Student Branch, Mansoura University, Egypt

\section{Technical Skills}

Programming:

MatLab, C/C++, OpenCV

\section{Languages:}

Arabic (Native), English (Fluent)

\section{References}

Available upon request 